(2) (1) a $13 y$
10

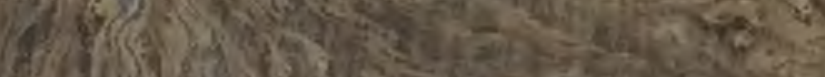
(2) 1 .

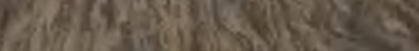

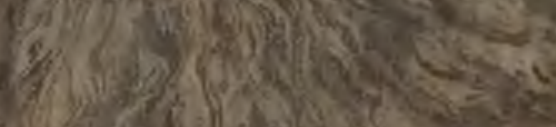

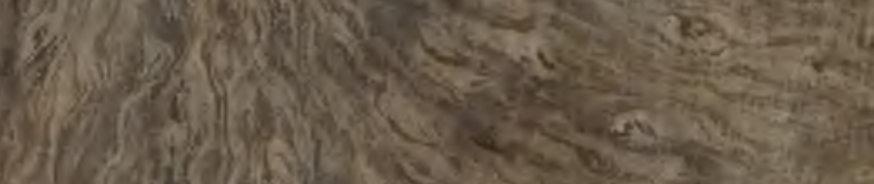

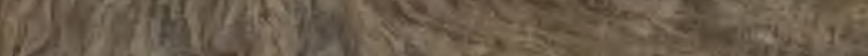

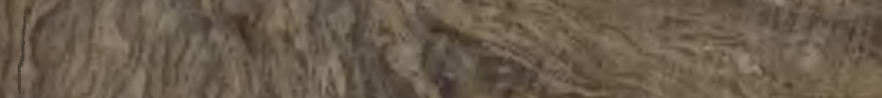

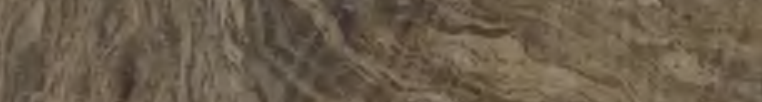

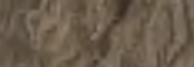

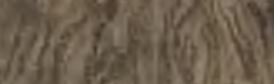

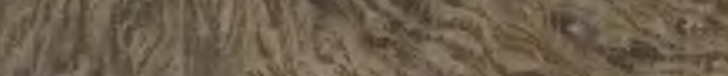

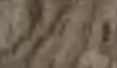
$(3)$

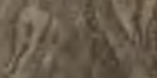
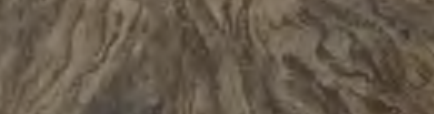

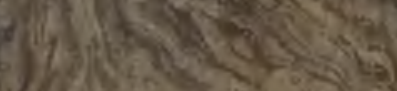

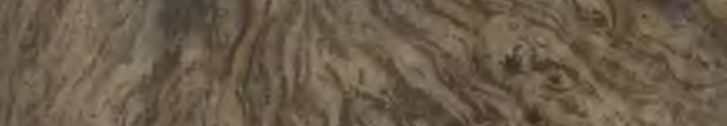

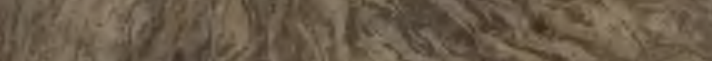

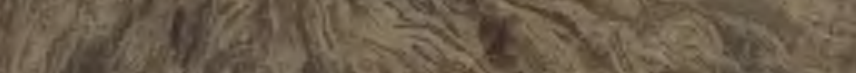

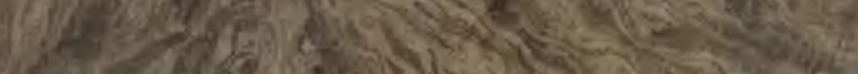

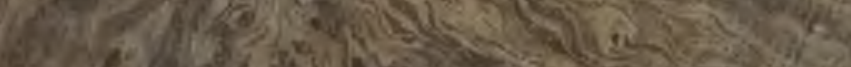

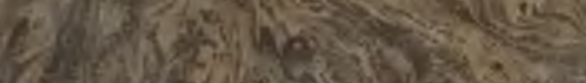




\section{$M_{1 t}$}

\section{Z Biblioteki}

c. K.

\section{OBSERWA'TORIUM} astronomicznego w KRA KO W I E.

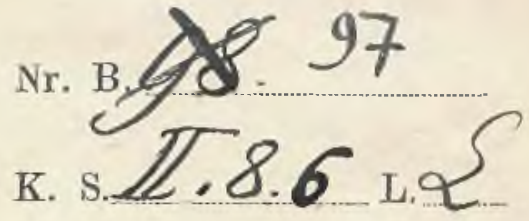






\section{ESSAI PHILOSOPHIQUE}

SUR.

LES PROBABILITES 


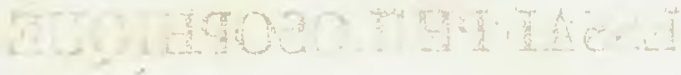

$$
\begin{aligned}
& +202+2
\end{aligned}
$$

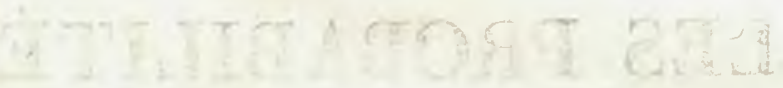




\title{
ESSAI PHILOSOPHIQUE
}

\author{
SUR
}

\section{LES PROBABILITÉS;}

\section{PAR M. LE COMTE LAILACE,}

Pair de France; Grand-Officier de la Legion-d'Honuenr; Grand'Croir de l'Ordre de la Réunion; Nembre de l'Institut royal et du Burean des Longitudes de France; des Socićtés royales de Londres et de Gottinguc; des Académies des Sciences de Russie, de Danemarck, de Suèdc, de Prusse, d'Italic, ete.

\section{SECONDE ÉDITION,}

REYUe et augmenté par L'AUteur.

\section{PARIS,}

Ame Ye COURCIER, Imprimcur-Liluraire pour les Mathématiques et la Marine, quai des Angustins, no 57 . 
$+\cdots+r+$

F
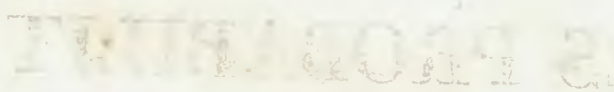

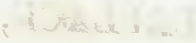

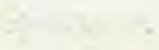

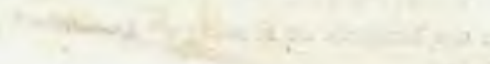

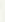

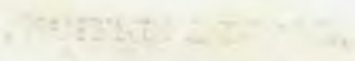

$=4+4+4=-2$ 


\title{
ESSAI PHILOSOPHIQUE
}

\author{
SUR
}

\section{LES PROBABILITES.}

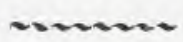

Cetr Essai philosophique est le développement d'une leçon sur les probabilités, que je donnai en $179^{5}$, aux écoles normales où je fus appelé comme professeur, par un décret de la Convention nationale. J'ai publié depuis peu sur le mème sujet, un ouvrage ayant pour titre: Théorie analytique des probabilités. Je présente ici, sans le secours de l'analyse, les principes et les résultats généraux de cette théorie, en les appliquant aux questions les plus importantes de la vie, qui ne sont en effet, pour la plupart, que des problemes de probabilité. On peut même dire, à parler en rigueur, que presque toutes nos conmaissances ne sont que probables; et dans le petit nombre des choses que nous pouvons savoir avec certitude, dans les sciences mathématiques ellesmêmes, les moyens de parvenir ia la vérité, sont fondés sur les probabilités; ensorte que le systime entier des connaissances humaines, 
se rattache à la thćorie exposée dans cet essai. On y verra sans doute avec intérêt, qu'en ne considérant mème dans les principes éternels de la raison, de la justice et de l'humanité, que les chances heureuses qui leur sont constamment attachées; il y a un grund avantage. à suivre ces principes, et de graves inconvéniens à s'en écarter: leurs chances, comme celles qui sont favorables aux loteries, finissant toujours par prévaloir au milieu des oscillations du hasard. Je desire que les réflexions ripandues dans cet essai, puissent mériter l'attention des philosophes, et la diriger vers un objet si digne de les occuper.

\section{De la Probabilité.}

Tous lesévénemens, ccux méme qui parleur petilesse, scmblent ne pas tenir aux grandes lois de la nature, en sont une suite aussi nécessaire que les révolutions du soleil. Dans l'ignorance des liens qui les unissent au système entier de l'univers, on les a fait dépendre des causes finales, ou du hasard, suivant qu'ils arrivaient et se succédaient avec régularité, ou sans ordre apparent; mais ces causes imaginaires ont été successivement reculées avec les bornes de nos connaissances, et disparaissent entièrement devant la saine philosophie qui ne voit en elles, que l'expression de 


\section{SUR LES PROBABTLITÉS.}

l'ignorance ou nous sommes des véritables caluses.

Les événemens actuels ont avec les précédens, une liaison fondée sur le principe évident, qu'une chose ne peut pas commencer d'être, sans une cause qui la produise. Cet axiome connu sous le nom de principe de la raison suffisanle, s'étend aux actions même les plus indifférentes. La volonté la plus libre ne peut saus un motif déterminant, leur donner naissance; car si toutes les circonstances de deux posilions étant exactement les mêmes, elle agissait dans l'une et s'abstenail d'agir dans l'autre, son choix serait un effet sans cause : elle scrait alors, dit Leibnitz, le hasard aveagle des épicuriens. L'opinion contraire est une illusion de l'esprit qui perdant de vue, les raisons fugitives du choix de la volonté dans les choses indifférentes, se persuade qu'elle s'est déterminée d'clle-même et sans motifs.

Nous devons donc envisager l'état présent de l'univers, comme l'effet de son état anléricur, et comme la cause de celui qui va suivre. Une intelligence qui pour un instant donné, connaîtrait toutes les forces dont la nature est animée, et la situation respective des êtres qui la composent, si d'ailleurs elle était assez vaste pour soumettre cés données à l'analyse, embrasserait dans la mêmo 
formule, les mouvemens des plus grands corps de l'univers et ceux du plus léger atome : rien ne scraitincertain pour elle, etl'avenir comme le passé, serait présent à ses yeux. L'esprit. humain offre dans la perfection qu'il a su donner à l'astronomie, une faible esquisse de cette intelligence. Ses découvertes en mécanique et en géométrie, jointes à celle de la pesanteur universelle, l'ont mis à portće de comprendre dans les mêmes expressions analytiques, les étals passés et futurs du système du monde. En appliquant la même méthode à quelques autres objets de ses connaissances, il est parvenu à ramener à des lois générales, les phénomènes observés, et à prévoir ceux que des circonstances données doivent faire éclore. Tous ses efforts dans la recherche de la vérité, tendent à le rapprocher sans cesse de l'intelligence que nous venons de concevoir, mais dont il restera toujours infiniment éloigné. Cette tendance propre à l'espèce humaine, est ce qui la rend supérieure aux animaux; et ses progrès en ce genre, distinguent les nations et les siecles, et fondent leur véritable gloire.

Rappelons-nousqu'autrefois età une époque qui n’est pas encore bien reculée, une pluie ou une sécheresse extrême, une comète traînant après elle une queuc fort étendue, les éclipses, les aurores boréales et généralement 
tous les phénomènes extraordinaires étaien regardés comme autant de signes de la colćre céleste. On invoquait le ciel pour détourner leur funeste influence. On ne le priait point de suspendre le cours des planètes et du soleil: l'observation eùt bientôt fait sentir l'inutilité de ces prières. Mais parce que ces phénomènes arrivant et disparaissantà de longs intervalles, semblaient contrarier l'ordre de la nature; on supposait que le ciel les faisait naitre et les modifiait à son gré, pour punir les crimes de la terre. Ainsi la longue queue de la comète de 1456 répandit la terreur dans l'Europe, déja constcrnée par les succès rapieles des Turcs qui venaient de renverser le Bas-Empire; et le pape Callixte ordonna des prières publiques dans lesquelles on conjurait la comète et les Turcs. Cet astre, après quatre de ses révolutions, a excité parmi nous un intérêt bien différent. La connaissance des lois du système du monde, aequise dans cet intervalle, avail dissipé les craintes enfantées par l'ignorance des vrais rapports de l'homme avec, l'univers; et Halley ayant reconnu l'identité de la comète, avec celles des années $153 \mathrm{I}$, 1607 et 1682 , il annonça son prochain retour pour la fin de 1758 ou le commencement de $175 \mathrm{~g}$. Le monde savant attendit avec impatience, ce retour qui devait confirmer l'uno. 
des plus grandes découvertes que l'on eût faites dans les sciences, et accomplir la prédiction de Sénèque, lorsqu'il a dit en parlant de la révolution de ces astres qui descendent d'une énorme distance : « Le jour viendra que par 3) une étude suivic de plusicurs siceles, les " choses actuellement cachées paraîlrontayce » évidence, et la postérité s'étonnera que des » vérilés si claires nous aient échappé.» Clairaut entreprit alors de soumettre à l'analyse, les perturbations que la comète avait éprouvées par l'action des deux plus grosses planètes, Jupiter et Saturne: après d'immenses calculs, il fixa son prochain passage au périhélic, vers le commencement d'avril 1759 , ce que l'observation ne tarda pas à vérifier. La régularité que l'astronomic nous montre dans le mouvemenl des cométes, a lieu sans aucun doute, dans tous les phénomènes. La courbe décrite par une simple molécule d'air ou de vapeurs, est réglée d'une manière aussi certaine, que les orbites planétaires : il n'y a de différence entre elles, que celle qu'y met notre ignorance.

La probabilité est relative en partie à cette jonorance, et en partic a nos connaissances. Nous savons que sur trois ou un plus grand nombre d'événemens, un seul doit arriver; mais rien ne porle à croire que l'un d'eux 
arrivera plutôt que les autres. Dans cet élat d'indécision, il nous est impossible de prononcer avec certitude sur leur arrivée. Il est cependant probable qu'un de ces évênemens pris à volonté, n'arrivera pas; parce que nous voyons plusieurs cas également possibles qui excluent son existence, tandis qu'un seul la favorise.

La thćorie des hasards consiste à réduire tous les événemens du même genre, à un certain nombre de cas également possibles, c'esta-dire, tcls que nous soyons également indécis sur leur existence; et à déterminer le nombre des cas favorables à l'événement dont on cherche la probabilité.Le rapport de ce nombre à celui de tous les cas possibles, est la mesure de celte probabilité qui n'est ainsi qu'une fraction dont le numérateur est le nombre des cas favorables, et dont le dénominateur est le nombre de tous les cas possibles.

La botion précédente de la probabilité suppose qu'cn faisantcroître dans lemême rapport, le nombre des cas favorables, et celui de tous les cas possibles, la probabilité reste la même. Pour s'en convaincre, que l'on considère deux urnes $\mathrm{A}$ et $\mathrm{B}$, dont la première conticnne quatre boules blanches et deux noires, et dont la seconde ne renferme que deux boules blanches et une noire. On peut imaginer ies 
deux boules noires de la première urne, at-tachées à un fil qui se rompt au moment oư l'on saisit l'une d'elles, et les quatre boulcs blanches formant deux systèmcs semblables. Toutes les chances qui feront saisir l'une des boules du système noir, amèneront une boulc noire. Si l'on conçoit maintenant que les fils qui unissent les boules, ne se rompent point; il est clair que le nombre des chances possibles ne changera pas, non plus que celui des chances firvorables à l'extraction des boules noires; seulement, on tirera de l'urne, deux boules à-la-fois; la probabilité d'extraire une boule noire de l'urne, sera donc la même qu'auparavant. Mais alors, on a évidemment le cas de l'urne $B$, avec la seule différence, que les trois boules de cette dernière urne, sont remplacées par trois systèmes de deux boules invariablement unies. Ici les cas également possiblesne sont pas les extractions desboules; ce sont les chances qui les amènent et dont la somme supposée la même pour chaque urne, est répartie sur six boules dans la première, et sur trois dans la seconde.

Quand tous les cas sont favorables à un événcment, sa probabilitó se change en certitude, et son expression devient égale à l'unité. Sous ce rapport, la certitude ct la probabilitẹ sont comparables, quoiqu'il y ait une 
différence esscntielle entre les deux états de l'esprit, lorsqu'une vérilé lui est rigoureusement démontrée, ou lor'squ'il aperçoit encore une petite source d'erreur.

Dans les choses qui ne sont que vraisemblables, la difference des données que chaque homme a sur elles, est une des causes principales de la diversité des opinions que l'on voit régner sur les mêmes objets. Supposons, par exemple, que l'on ait trois urnes $A, B, C$, dont l'une ne contienne que des boules noires, tandis que les deux autres ne renferment que des boules blanches. On doit tirer une boule de l'urne $\mathrm{C}$, et l'on demande la probabililé que celte boule scra noire. Si l'on ignore quelle est celle des trois urnes, qui ne renferme que des boules noires, ensorte que l'on n'ait aucune raison de croire qu'elle est plutòt C, que Bou A; ces trois hypothèses paraîtront également possibles; et comme une boule noire ne peut être extraite que dans la première, la probabilité de l'extraire est égale à un tiers. Si l'on sait que l'urne A ne contient que des boules blanches, l'indécision ne porte plus alors que sur les urnes $\mathrm{B}$ et $\mathrm{C}$, et la probabilité que la boule extraite de l'urne C sera noire, est un demi. Enfin cette probabilité se change en certitude, si l'on est assuré que les 
urnes $\mathbf{A}$ et $\mathbf{B}$ ne contiennent que des boules blanches.

Cest ainsi que le même fait récité devant une nombreuse assemblée, obtient divers degrés de croyance, suivant l'élendue des connaissances des auditeurs. Si l'homme qui le rapporte, en est intimement persuadé, et si par son état et son caractère, il inspire une grande confiance; son récit, quelqu'extraordinaire qu'il soit, aura par rapport aux auditeurs dépourvus de lumiéres, le même degré de vraisemblance, qu'un fait ordinaire rapporté par le même homme, et ils Iui ajouteront unc foi entière. Cependant si quelgu'un d'eux a cu occasion d'entendre le même fait rejeté par d'autres hommes également respectables, il sera dans le doute; et le fait sera jugé faux, par les auditeurs éclairés qui le trouveront contraire, soit à des faits bien avérés, soit aux lois immuables de la nature.

C'est à l'influence de l'opinion de ceux que la multitude juge les plus instruits, el à qui elle a coutume de donner sa confiance sur les plus importans objets de la vie, qu'est due la jropagaíion de ces erreurs qui, dans les temps dignorance, ont couvert la face du monde. L'astrologie nous en offre un grand exemple. Ces erreurs inculquées dés l'enfance, adoptíes 


\section{SUR LES PROBABITITES.}

sans examen, et n'ayant pour base que la croyance universelle, se sont maintenues pendant très-long-temps; jusqu’a ce qu'enfin le progrès des scicnces les ait détruites dans l'esprit des hommes éclairés, dont ensuite l'opinion les a fait disparaitre chez le peuple mểme, par le pouvoir de l'imitation et de l'ha. bitude, qui les avaitsigénéralementrépandues. Ce pouvoir, le plus puissant ressort du monde moral, établit et conserve dans toute une nation, des idées entièrement contraires à celles qu'il maintient ailleurs avec le même empire. Quelle indulgence ne devons-nous donc pas a voir pour les opinions différentes des nôtres; puisque cette différence ne dépend souvent que des points de vue divers ou les circonstances nous ont placés! Éclairons ceux que nous ne jugeons pas suffisamment instruits; mais auparavant, examinons sévèrement nos propres opinions, et pesons avec impartialité, leurs probabilités respectives.

$\mathrm{La}$ différence des opinions dépend encore de la manière dont chacun détermine l'influence des données qui lui sont connues. La théorie des probabilités tient à des considérations si délicates, qu'il n'est pas surprenant qu'avec les mêmes donnćes, deux personnes trouvent des résultats diférens, surtout dans 
les questions très-compliquées. Exposons ici les principes généraux de cette théorie.

Principes généraux du Calcul des Probabilités.

Ier Principe. Le premier de ces principes cst la définition même de la probabilité qui, comme on l'a vu, est le rapport du nombre des cas favorables, à celui de tous les cas possibles.

11e Prinsipe. Mais cela suppose les divers cas, également possibles. S'ils ne le sont pas, on déterminera d'abord leurs possibilités respectives dout la juste appréciation est un des points les plus délicats de la théorie des hasards. Alors la probabilité sera la somme des possibilités de chaque cas favorable. Éclaircissons ce principe par un exemple.

Supposons que l'on projette en l'air, une pièce large et tris-mince dont les deux grandes faces opposces, que nous nommerons croix et pile, soient parfaitement semblables. Cherchons la probabilité d'amener croix, une fois au moins en deux coups. Il est clair qu'il peut arriver quatre cas également possibles, savoir, croix au premier et au second coup; craix au premier coup et pile au second; pile au premier coup et croix au second; enfin pile 
aux deux coups. Les trois premiers cas sont favorables à l'événement dont on cherche la probabilité qui, par conséquent, est égale à $\frac{3}{4}$; ensorte qu'il y a tıois contre un à parier que croix arrivera au moins une fois en deux coups.

On peut ne compter i ce jeu, que trois cas différens, savoir, croix au premier coup, ce qui dispense d'en jouer un second; pile au premier coup et croix au second; enfin pile au premier et au second coup. Cela réduirait la probabilité à $\frac{2}{3}$, si l'on considérait avec d'Alembert, ces trois cas, comme étant également possibles. Mais il est visible que la probabilité d'amener croix au premier coup est $\frac{1}{2}$, tandis que celle des deux autres cas est $\frac{1}{4}$. Le premier cas est un événement simple qui correspond aux deux ćvénemens composés, croix au premier et au second coup, et croix au premier coup, pile au sccond. Maintenant, si conformément au second principe, on ajoute la possibilité $\frac{1}{2}$ de croix au premier coup, à la possibilité $\frac{1}{4}$ de pile arrivant au premier coup et croix au second; on aura $\frac{3}{4}$ pour la probabilité cherchéc, ce qui s'accorde avec ce que l'on trouve dans la supposition oúk lon joue les deux coups. Cette supposition ne change ricn au sort de celui qui parie pour cet événement: clle sert seulement à 
réduire les divers cas, à des cas également possibles.

LII Principe.

Un des points les plus importans de la Théorie des Probabilités, et celui qui prête le plus auxillusions, est la manière dont les probabilités augmentent ou diminuent par leurs combinaisons muluelles. Si les événemens sont indépendans les uns des autres, la probabilité de l'existence de leur ensemble, est le produit de leurs probabilités particulières. Ainsi la probabilité d'amener un as avec un seul dé, étant un sixième; celle c'amener deux as en projetant deux dés à-la-fois, est un trentesixième. En effet, chacune des faces de l'un, pouvant se combiner avec les six faces de lautre; il y a trente-six cas également possibles, parmi lesquels un seul donne les deux as. Généralement, la probabilité qu'un événement simple et dans les mêmes circonstances, arrivera de suite, un nombre donné de fois, est égale à la probabilité de cet événement simple, élevée à une puissance indiquée pan ce nombre. Ainsi les puissances successives d'une fraction moindre que l'unité, diminuant sans cesse; un événement qui dépend d'une suite de probabilités fort grandes, peut devenir extrêmement peu vraisemblable. Supposons qu'un fait qui sans être extraordinaire, n'a aucune probabilité par lui-même, nous soil 


\section{SUR LES PROT̈BARTITÉS.}

transmis par vingt tćmoins, de maniče que le premier l'ail transmis au second, le second au troisieme, et ainsi de suite. Supposons encore que la probabilité de chaque témoignage soit égalc à $\frac{9}{10}$ : cellc du fait sera moindre qu'un huiticme; c'est-it-dire qu'il y aura plus de sept à parier contre un, qu'il est faux. On ne peut micux comparer cette diminution de la probabilité, qu'á l'extinction de la clarté des objets, par l'interposition de plusieurs morceaux de verre; un nombre de morceaux peu considérable, suffisant pour dérober la vue d'un objet quin seul morceau laisse apcrcevoir d'une manicre distincte. Los historiens ne pam raissent pas avoir fait assez d'altention à cette dégradation de la probabilité des faits, Jorsqu'ils sont vus à travers un grand nombre de générations successives: plusieurs événemens hisloriques, réputés certains, seraientau moins douteux, si on les soumettait ì cette épreuve.

Dans les sciences purement mathématiques les conséquences les plus éloignées participent de la certitude da principe dont elles dérivent. Dans les applications de l'analyse à la physique, les conséquences ont toute la certitude des faits ou des expériences. Mais dans les sciences morales, où chaque conséquence n'est déluite de ce qui la précède, que d'une manière vraisemblable; queiqueprobables que 
soient ces déductions, la chance de l'erreur croit avec leur nombre, et finit par surpasser la chance de la vérité, dans les conséquences très-éloignées du principe.

incipe. Quand deux événemens dépendent l'un de l'autre; la probabilité de l'événement composé est le produit de la probabilité du premier événement, par la probabilité que cet événement étant arrivé, l'autre aura lieu. Ainsi, dans le cas précédent de trois urnes $\mathrm{A}, \mathrm{B}, \mathrm{C}$, dont deux ne contiennent que des boules blanches, et dont une ne renferme que des boules noires; la probabilité de tirer une boule blanche de l'urne C est $\frac{2}{3}$; puisque sur trois urnes, deux ne conliennent que des boules de cette conleur. Mais lorsqu'on a extrait une boule blanche, de l'urne C; I'indécision relátive a celle des urnes qui ne renferme que des boules noires, ne portant plus que sur les urnes $A$ et $B$; la probabilité d'extraire une boule blanche, de l'urne B est $\frac{x}{2}$; le produit de $\frac{2}{3}$ par $\frac{1}{2}$, ou $\frac{1}{3}$ est done la probabilité d'extraire à-la-fois des urnes B et $\mathrm{C}$, deux boules blanches.

On voit par cet exemple, l'influence des événemens passés sur la probabilité des événemens futurs. Car la probabilité d'extraire une boule blanche, de l'urne B, qui primitivement est $\frac{2}{3}$, se réduit à $\frac{1}{2}$, lorsqu'on a extrait 
une boule blanche, de l'urne $\mathrm{C}$ : elle se changerait en certitude, si l'on avait extrait une boule noire, de la même urne. On déterminera cetteinfluence, aumoyen du principe suivant, qui cst un corollaire du précédent.

Si l'on calcule à priori, la probabilité de ve Principe. l'événement arrivé, et la probabilité d'un événement composé de celui-ci et d'un autre qu'on attend; la seconde probabilité divisée par la première, sera la probabilité de l'événement attendu, tirée de l'événement observé.

Icise présentela questionagitée par quelques philosophes, touchant linfluence du passé sur Ia probabilité de l'avenir. Supposons qu'au jeu de croix et pile, croix soit arrivé plus souvent que pile. Par cela seul, nous serons portés à croire que dans la constitution de la pièce, il existe une cause constante qui le favorise. Ainsi, dans la conduite de la vie, le bonheur constant est une preuve d'habileté, qui doit faire employer de préférence les personnes heureuses. Mais si par l'instabilité des circonstances, nous sommes ramenés sans cesse, ̀̀ l'état d'uneindécision absolue; si, par exemple, on change de pièce à chaque coup, au jeu de croix et pile; le passé ne peut répandre aucune lumière sur l'avenir, et il serait absurde d'en tenir compte. 
incipe. Chacune des causes auxquelles un événement observé, peut être attribué, est inảiquée avec d'autant plus de vraisemblance, qu'il est plus probable que cette cause étant supposće exister, l'événement aura lieu; la probabilité de l'existence d'une quelconque de ces causes, est donc une fraction dont le numérateur est la probabilité de l'événement, résultante de cette cause, et dont le dénominateur est la somme des probabilités semblables relatives à toutes les causes: si ces diverses causes considérćes à priori, sont inégalement probables; il faut an lieu de la probabilité de l'événement, résultante de chaque cause, employer le produit de cette probabilité, par celle de la cause elle-même. C'est le principe fondamental de cette branche de l'analyse des hasards, qui consiste à remonter des événemens aux causes.

Ce principe donne la raison pour laquelle on attribue les événemens réguliers, à une cause particulière. Quelques philosophes ont cru que ces événemens sont moins possibles que les autres, et qu'au jeu de croix et pile, par exemple, la combinaison dans laquelle croix arrive vingt fois de suite, est moins facile à la nature, que celles ou croix et pile sont entre-mêlés d'une façon irrégulière. Mais cette opinion suppose que les événemens passés 


\section{SUR LES PROBABILITÉs.}

infiuent sur la possibilité des événemens futurs, ce qui n'est point admissible. Les combinaisons régulières n'arrivent plus rarement, que parce qu'elles sont moins nombreuses. Si nous recherchons une cause, lì où nous apercevons de la symétric; ce n'est pas que nous regardions un événement symétrique, comme moins possible que les autres; mais cet événement devant être l'effet d'une cause régulière, ou celui du hasard, la première de ces suppositions est plus probable que la seconde. Nous voyons sur une table, des caractères d'imprimerie, disposés dans cet ordre, Constantinople; et nous jugeons que cet arrangemont n'est pas l'effet du hasard, non parce qu'il cst moins possible que les autres, puisque si ce mot n'était employé dans aucune langue, nous ne lui soupçonnerions point de cause particulière; mais ce mot étant en usage parmi nous, il est incomparablement plus probable qu'une personne aura disposé ainsi les caractères précédens, qu'il ne l'est que cet arran- . gement est dù au hasard.

C'est ici le licu de détinir le mot extraordinaire. Nous rangeons par la penséc, tous les événemens possibles, en diverses classes; et nous regardons comme extraordinaires, ceux des classes qui en comprennent un trèspetit nombre. Ainsi, au jeu de croix et pile, 
l'arrivée de croix cent fois de suite, nous parait extraordinaire, parce que le nombre presqu'infimi des combinaisons qui peuvent arriver en cent coups, étanit partagé en séries régulières ou dans lesquelles nous voyons réguer un ordre facile à saisir, et en séries irrégulic̀res; celles-ci sont incomparablement plus nombreuses. La sortie d'une boule blanche, d'une urne qui, sur un million de boules, n'en contient qu'une seule de cette couleur, les autres étant noires, nous paraît encore extraordinaire; parce que nous ne formons que deux classes d'événemens, relatives aux deux couleurs. Mais la sortie du n 79 , par exemple, d'une urne qui renferme un million de numéros, nous semble un événement ordinaire; parce que comparant individuellement les numéros, les uns aux autres, sans les partager en classes, nous n'avons aucune raison de croire que l'un d'eux sortira plutôt que les autres.

De ce qui précède, nous devons généralement conclure que plus un fait est extraordinaire, plus il a besoin d'être appuyé de fortes preuves. Car ceux qui l'attestent, pouvant ou tromper, ou avoir été trompés, ces deux causes sont d'autant plus probables, que la réalité du fait l'est moins en elle-même. C'est ce que l'on verra particulièrement, lorsque 


\section{SUR LES PROBABILITÉS.}

nous parlerons de la probabilité des témoignages.

La probabilité d'un événement futur est vi[eP la somme des produits de la probabilité de chaque cause, tirée de l'événement observé, par la probabilité que cette cause existant, l'événement futur aura lieu. L'exemple suivant éclaircira ce principe.

Imaginons une urne qui ne renferme que deus boules dont chacune soit ou blanche, ou noire. On extrait une de ces boules, que l'on remęt ensuite dans l'urne, pour procéder à un nouveau tirage. Supposons que dans les deux premiers tirages, on ait amené des boules blanches; on demande la probabilité d'amener encore une boule blanche au troisieme tirage.

On ne peut faire ici que ces deux hypothèses; ou l'une des boules est blanche, et l'autre, noire; ou toutes deux sont blanches. Dans la preinière hypothise, la probabilité de l'événement observé est $\frac{1}{4}$; elle est l'unilé ou la certilude dans la seconde. Ainsi, en regardant ces hypotheses, comme autant de causes, on aura par le sixième principe, $\frac{1}{5}$ et $\frac{4}{5}$ pour leurs probabilités respectives. Or si la première hypothèsea lieu, la probabilité d'exiraire une boule blanche au troisieme tirage est $\frac{\tau}{2}$; elle égale l'unité, dans la seconde hypothise: en muitipliant ces dernières probabilités, pax 
celles des hypothèses correspondantes, la somme des produits, ou $\frac{9}{n}$ sera la probabilité d'extraire une boule blanche au troisième tirage.

Quand la probabilité d'un événement simple est inconnue, on peut lui supposer également toutes les valeurs depuis zéro jusqu'à l'unité. La probabilité de chacune de ces hypothèses, tirée de l'événement observé, est par le sixième principe, une fraction dont le numéraleur est la probabilité de l'événement dans cette hypo. thèse, et dont le dénominateur est la somme des jrobabilités semblables relatives à toutes les hypothèses. Ainsi la probabilité que la possibilité de l'événement est comprise dans des limites données, est la somme des fractions comprises dans ces limites. Maintenant, si l'on multipie chaque fraction, par la probabilité de l'événement futur, déterminée dans l'hypothèse correspondante; la somme des produits relatifs à toutes les hypothèses sera par le septième principe, la probabilité de l'événement futur, tirée de l'événement $o b-$ servé. On trouve ainsi qu'un événement étant arrivé de suite, un nombre quelconque de fois; la probabilité qu'il arrivera encore la fois suivante, est égale à ce nombre augmenté de l'unité, divisé par le même nombre augmenté de deux unités. En faisant, par exemple, 
remonter la plus ancienne époque de l'histoire, à cinq mille ans, ou à 1826213 jours, et le soleil s'étant levé constamment dans cet intervalle, à chaque révolution de vingt-quatre heures; il y a 1826214 à parier contre $11 n$, qu'il se levera encore demain. Mais ce nombre est incomparablement plus fort pour celui qui connaissant par l'ensemble des phénomìnes, le principe régulateur des jours et des saisons, voit que rion dans le moment actuel, ne peut en arrêter le cours.

Buffon, dans son Arithmétique politique, calcule différemmentla probabilitéprécédente. Il suppose qu'elle ne diffère de l'unité, que d'une fraction dont le numérateur est l'unité, ct dont le dénominateur est le nombre denx élevé à une puissance égale au nombre des jours écoulés depuis l'ćpoque. Mais la vraie manic̀re de remonter des événemens passés, à la probabilité des causes et des évé-nemens futurs, était inconnue à cet illustre écrivain.

\section{De l'Espérance.}

La probabilité des événemens sert à déterminer l'espérance ou la crainte des personnes intéressées à leur existence. Le mot espérance a diverses acceptions : il exprime gé- 
néralement l'avantage de celui qui attend un bien quelconque, dans des suppositions qui ne sont que probables. Cet avantage, dans la théorie des hasards, est le produit de lasomme espérée, par la probabilité de l'obtenir : c'est la somme partielle qui doit revenir, lorsqu'on ne veut point courir les risques de l'événement, en supposant que la répartition se fasse proportionnellement aux probabilités. Celte répartition est la seule équitable, lorsqu'on fiit abstraction de toutes circonstances étrangères; parce qu'avec un égal degré de probabilité, on a un droit égal sur la somme espérée. Nous nommerons cet avantage, espérance mathématique.

ncipe. Lorsqu'il dépend de plusieurs événemens; on l'obtient, en prenant la somme des produits de la probabilité de chaque événement, par le bien attaché à son arrivée.

Appliquons ce principe à des exemples. Supposons qu'au jeu de croix et pile, Paul reçoive deux francs, s'il amène croix au premier coup, et cing francs, s'il ne l'amène qu'au second. En multipliant deux francs, par la probabilité $\frac{1}{2}$ du premier cas, et cinq francs, par la probabilité $\frac{1}{4}$ du second cas; la somme des produits, ou deux francs et un quart sera l'avantage de Paul. C'est la somme qu'il doit donner d'a vance à celui quilua fait cetavantage; 
car poür l'égalité du jeu, la mise doit être égale à l'avantage qu'il procure.

Si Paul reçoit deux francs, en amenant crotx au premier coup, et cinq francs en l'amenant au second coup, soit qu'il l'ait ou non, amené au premier; alors la probabilité d'amener croix au second coup, étant $\frac{1}{2}$; en multipliant deux francs et cinq francs par $\frac{t}{2}$, la somme de ces produits, donnera trois francs et demi pour l'avantage de Paul, et par conséquent pour sa mise au jeu.

Dans une série d'événemens probables, IXe Princips. dont les uns produisent un bien, et les autres, une perte; on aura l'avantage qui en résulte, en faisant une somme des produits de la probabilité de chaque événement favorable, par le bien qu'il procure; et en reiranchant de cette somme, celle des produits de la probabilité de chaque événemcnt délavorable, par la perte qui y est attachée. Si la seconde somme l'emporte sur la première, le bénéfice devient perte, et l'espérance se change en crainte.

On doit toujours, dans la conduite de la vie, faire ensorte d'égaler au moins, le produit du bien que l'on espère, par sa probabilité, au produit semblable relatif à la perte. Mais il est nécessaire pour y parvenir, d'apprécier exaclement les avantages, les pertes, et 
leurs probabilités respectives. II faut pour cela, une grande justesse d'esprit, un tact délicat, et une grande expérience des choses: il faut savoir se garantir des préjugés, des illusions de la crainte et de l'espérance, et de ces fausses idées de fortune et debonheur, dont la plupart des hommes bercent leur amourpropre.

L'application des principes précédens, à la question suivante, a beaucoup exercé les géomètres. Paul joue à croix et pile, avec la condition de recevoir, deux francs, s'il amène croix au premier coup; quatre francs, s'il ne l'amene qu'au second; huit francs, s'il ne l'amène qu'au troisième, et ainsi de suite. Sa mise au jeu, doit être par le huitième principe, égale au nombre des coups; ensorte que si la partie continue à l'infini, la mise doit être infinie. Cependant, aucun homme raisonnable ne voudrait exposer à ce jeu, une somme même modique, cinquantefrancs, par exemple. D'où vient cette différence entre le résultat du calcul, et lindication du sens commun? On reconnut bientôt, qu'elle tenait à ce que l'avantage moral qu'un bien nous procure, n'est pas proportionnel à ce bien, et qu'il dépend de mille circonstances souvent très-difficiles à définir, mais dont la plus générale et la plus importante est celle de la fortune. En efret, 
il est visible qu'un franc a beaucoup plus de prix pour celui qui n'en a que cent, que pour un millionnaire. On doit donc dans le bien espéré, distinguer sa valeur absolue, de sa valeur relative. Celle-ci se règle sur les motifs qui le font desirer; au lieu que la première en est indépendante. On ne peut pas donner de principe général, pour apprécier celte valeur relative. En voici cependant un proposé par Daniel Bernoulli, et qui peut servir dans beaucoup de cas.

La valcur relative d'une somme infiniment Xe Principe. petite, est égale à sa valeur absolue diviséc par le bien total de la personne intéressée. Cela suppose que tout ho:nme a un bien quelconque dont la valeur ne peut jamais être supposée nulle. En offet, celui même qui ne possède rien, donne toujours à son existence, une valcur au moins égale à ce qui luiest rigouretisement nécessaire pour vivre.

Si l'on applique l'analyse, au principe que nous venons d'exposer; on obtient la règle suivante.

En désignant par l'unité, la partie de la fortune d'un individu, indépendante de ses expectatives; si l'on détermine les diverses valeurs que cette fortune peut recevoir en vertu de ces expectatives, et leurs probabilités; le produit de ces valeurs úlevées respectivement 
aux puissances indiquées par ces probabilités, sera la fortune physique qui procurerait à lindividu, le même avantağe moral qu'il reçoit de la partie de sa fortune, prise pour unité, et de ses expectatives; en retranchant donc l'unité, de ce produit ; la différence sera l'accroissement de la fortune physique, dî aux expectatives : nous nommerons cet accroissement, espérance morale. Il est facile de voir qu'elle coïncide avec l'espérance mathématique, lorsque la fortune prise pour unité, devient infinie par rapport aux variations qu'elle reçoit des expectatives. Mais lorsque ces variations sont une partie sensible de cette unité, les deux espérances peuvent différer très-sensiblement entre elles.

Cette règle conduità des résultats conformes aux indications du sens commun, que l'on peut à ce moyen, apprécier avec quelqu'exactitude. Ainsi dans la question précédente, on trouve que si la fortune de Paul est de deux cents francs, il ne doit pas raisonnablement mettre au jeu plus de neuf francs. La même règle conduit encore à répartir le danger, sur plusieurs parties d'un bien que l'on espère, plutòt que d'exposer ce bien tout entier au même danger. Il en résulte pareillement, qu'au jeu le plus égal, la perte est toujours relativement plus grande que le gain; 
car le produit de la fortune prise pour unité, augmentée du gain et élevée à une puissance égale à la probabilité da gain, par cette unité diminuée de la perte, et ćlevéeà une puissance égale à la probabilité de la perte, est toujours moindre que la fortune du joueur avant sa mise au jeu. En supposant par exemple, cette fortune, de cent francs, ct que le joueur en expose cinquante au jeu de croix et pile; sa fortune après sa mise au jeu, peut être en vertu de son expectative, ou de cent cinquante francs, ou seulement de cinquante: la probabilité de chacun de ces deux cas est $\frac{1}{5}$; cette fortune est donc par la règle précédente, égale à la racine carrée du prodait de cent cinquante, par cinquante; elle est ainsi réduite à quatre-vingt-sept francs, c'est-à-dire que cette dernière somme procurerait au joueur, le même avantage moral, que l'état de sa fortune après sa mise. Le jeu est done désavantageux, dans le cas même où la mise estégale au produit de la somme espérée, par sa probabilité. On peut juger par lì de l'immoralité des jeux dans lesquels la somme espérée est au-dessous de ce produit. Ils ne subsistent que par les fuux raisonnemens et la cupidité qu'ils fomentent, et qui portant le peuple à sacrifier son nécessaire, à des espérances chimériques dont il est hors d'état d'apprécier 
l'invraisemblance, sont la source d'une infinité de maux.

Des Méthodes analytiques du Calcul des Probabilités.

L'application des principes que nous venons d'exposer, aux diverses questions de probabilités, exige des méthodes dont la recherche a donné naissance à plusieurs branches de l'analyse, et spécialement à la théorie des combinaisons, et au calcul des différences finies.

Si l'on forme le produit des binomes, l'unité plus une première: letre, l'unité plus une seconde lettre, l'unité plus une troisicme lettre, et ainsi de suite jusqu'a $n$ lettres; en retranchant l'unité de ce produit développé, on aura la somme des combinaisons de toutes ces lettres prises une à une, deux à deux, trois à trois, etc. : chaque combinaison aura pour coefficient, l'unité. Pour avoir le nombre des combinaisons de ces $n$ lettres prises $r a \dot{r}$, on observera que si on suppose les lettres égales entre elles, le produit précédent deviendra la puissance $n^{i e m m e}$ du binome, un plus la première lettre; et le nombre des combinaisons des $n$ lettres prises $r$ à $r$, sera le coefficient de la puissance riène de la première letre, 
dans le développement de ce binome; on aura donc ce nombre, par la formule connue da binome.

Si l'on veut avoir égard à la situation respective des lettres, dans chaque combinaison; on doit observer qu'en joignant une seconde lettre à la première, on peut la placer au premier et au second rang; ce qui donne deux combinaisons. Si l'on joint à ces combinaisons, une troisième lettre; on peut lui donner dans chaque combinaison, le premier, le second et le troisieme rang; ce qui forme trois combinaisons relatives à chacune des deux autres, en tout, six combinaisons. De là, ir est aisé de conclure que le nombre des arrangemens différens que l'on peut donner à $r$ lettres, est le produit des nombres depuis l'unité jusqu'à $r$. Il faut donc pour avoir égard a la situation respective des lettres, mulliplier par ce produit, le nombre des combinaisons des $n$ lettres prises $r$ à $r$; ce qui revient à supprimer le dénominateur du coefficient du terme du binome, qui exprime ce nombre.

Supposons une lotcrie composée de $n$ numéros, et qu'il en sorte $r$ à chaque tirage; on demande la probabilitié de la sortie de $s$ numéros donnés, dans un tirage. Pour y parvenir, on déterminera d'abord le nombre des combinaisons des autres numéros pris $r$ moins 
$s$, à $r$ moins $s$; car il est clair qu'en ajoutant les $s$ numéros donnés, à chacune de ces combinaisons, on aura la somme de toutes les combinaisons des $n$ lettres prises $r \dot{a} r$, et dans lesquels les $s$ numéros donnés entrent. Si l'on divise ce nombre, par celui des combinaisons de toutes les lettres prises $r$ à $r$; on aura la probabilité demandée. On trouve ainsi que cette probabilité est le rapport du nombre des combinaisons de $r$ lettres prises $s$ à $s$, au nombre des combinaisons de $n$ lettres prises $s$ à $s$.

On peut d'après ce théorème, calculer les chances de la loterie de France, et en conclure ses bénélices. Celte loterie est, comme on sait, composée de go numéros, dont cinq sortent à chaque iirage. La probabilité de la sortie d'un extrait donné, est en vertu de ce théorème, égale à $\frac{5}{90}$ ou $\frac{1}{18}$; la loterie devrait donc alors pour l'égalité du jeu, rendre dixhuit fois la mise. Le nombre total des combinaisons deux à deux, de go numéros est 4005 , et il en sort dix à chaque tirage; ainsi la probabilité de la sortie d'un ambe donné est $\frac{10}{405}$; la loterie devrait donc pour un ambe sorti, rendre quatre cents fois et demie, la mise. On trouve pareillement qu'elle devrait rendre la mise, 11748 fois pour un terne, 511038 fois pour un quaterne, et 43949268 
fois pour un quine. La loterie est loin de faire ces avantages aux joueurs.

Supposons encore dans une urne, $n$ boules que l'on puisse également extraire une à une, deux à deux, trois à trois, etc.; on a fait une de ces extractions, et l'on demande la probabilité que le nombre des boules extraites est impair. Il suit de ce qui précède, que si l'on eleve le binome, un plus un, à la puissance $n$; les termes, second, troisiène, etc., cxprimeront les nombres de combinaisons des $n$ boules, prises une ì une, deux à deux, etc.; ainsi la totalité des combinaisons sera la puissance $n^{i c m}$ de deux, moins l'unité : la somme des termes second, qualrième, sixième, etc. du développement du binome, sera le nombre des combinaisons impaires : elle sera visiblement, la moilié de la différence dés $n^{i e m e}$ puissances des binomes un plus un, et un moins un; ou la moitié de la $n^{i e m e}$ puissance de deux. En retranchant l'unité, de celte quantité, on aura le nombre des combinaisons paires; et en divisant ces deux nombres de combinaisons, par leur somme, on aura les probabilités respectives des combinaisons impaires et paires. On voit ainsi qu'il y a de l'avantage à parier plutôt pour un nombre impair de boules extraites, que pour un nombre pair.

Mais la méthode la plus générale et la pius 
directe de résoudre les questions de probabilité, consiste à les faire dépendre d'équations aux différences. En comparant les états consécutifs de la fonction des variables, qui exprime la probabilité, lorsqu'on fait croître ces variables, de leurs différences respectives; la question proposée fournit le plus souvent, un rapport très-simple entre les divers états de cette fonction. Ce rapport est ce que l'on nomme équation aux différences ordinaires ou partielles; ordinaires, lorsqu'il n'y a qu'une variable; partielles, lorsqu'il y en a plusicurs. Donnons en quelques exemples.

Trois joueurs dont les forces sont supposées les mêmes, jouent ensemble aux conditions suivantes. Celui des deux premiers joueurs qui gagne son adversaire, joue avec le troisieme, et s'il le gagne, la partie est finie. Sil est vaincu, le vainqueur joue avec l'autre, et ainsi de suite, jusqu'à ce que l'un des joueurs ait gagné consécutivement les deux autres; ce qui termine la partie. On demande la probabilité que cette partie sera finie dans un nombre donné de coups. Cherchons d'abord la probabilité qu'elle finira précisément à un coup déterminé, par exemple, au dixième coup. Pour cela, le joueur qui la gagne, doit entrer au jeu au neuvième coup, et le gagner ainsi que le coup suivant. Mais si au lieu de 
gagner le neuvième coup, il était vaincu par son adversaire; comme celui-ci a déjà gagné lautre joueur, la partie finirait à ce coup; ainsi la probabilité qu'un joueur entrera au jeu au neuvième coup, et le gagnera, est égale à celle que la partie finira précisément à ce coup; et comme ce joueur doit gagner le coup suivant, pour que la partie se termine au dixièmecoup, cettedernière probabiliténesera qu'un demi de la précédente. Il suit de là que si l'on considère cette probabilité, comme une fonction du numéro du coup auquel elle doit finir; cette fonction sera la moitié de la même fonciion dans laquelle on a diminué le numéro ou la variable, d'une unité. Cette égalité forme une de ces équations que l'on nomme équations aux différences finies ordinaires.

On peut déterminer fucilement à son moyen, la probabilité que la partie finira précisément à un coup quelconque. Il est visible que la partie ne peut finir au plutôt, qu'au second coup; et pour cela, il est nécessaire que celui des deux premiers joueurs qui gagne son adversaire, gagne au second coup, le troisième joucur. Ainsi la probabilité que la partie finira à ce coup, est $\frac{1}{2}$. De là, en vertu de l'équation précédente, on conclut que les probabilités successives de la fin de la partie, soni $\frac{1}{4}$ pour lc troisième coup, $\frac{x}{8}$ pour le quatrième, etc., 
et généralement $\frac{x}{2}$ élevé à une puissance moindre de l'unité, que le numéro du coup. Maintenant, si l'on prend la somme de toutes ces puissances, depuis la premịre jusqu’à cette dernière inclusivement; on uura la probabilité que la partie sera terminée dans le nombre de coups indiqué par ce numéro, égale à l'unité moins la dernière de ces puissances de $\frac{t}{2}$.

Considérons encore le premier problème que l'on ait résolu sur les probabilités, ct que Pascal proposa de résoudre à Fermat. Denx joueurs $\mathbf{A}$ et $\mathbf{B}$, dont les adresses sont égales, jouent ensemble à cette condition que celui qui le premier, aura vaincu l'autre un nombre donné de fois, gagnera la partie, et emportera la somme des mises au jeu. Après quelques coups, les joueurs convieunent de se retirer sans avoir terminé la partie; on dèmande de quelle maniere ils doivent se partager cette somme. Il est visible qué leurs parts doivent être proportionnelles à leurs probabilités respectives de gaguer la partie; la question se réduit donc à détermincr ces probabilités. Elies dépendent évidemment des nombres de points qui manquent à chaque joueur, pour atteindre le nombre donné; ainsi la probabilité de $A$ est une fonction de ces deux nombres que nous regarderons comme autant de variables. 
Si les deux joneurs convenaient de jouer un coup de plus (convention qui ne change en rien leur sort ); ou A le gagnerait, et alors le nombre des points qui lui manque, serait diminué d'une unité; ou le joueur $B$ gagnerait ce nouveau coup, et alors le nombre des points qui manquent à ce dernier joueur, serait diminué d'une unité; mais la probabilité de chacun de ces cas est $\frac{1}{2}$; la fonction cherchée est donc égale à la moitié de cetie fonction dans laquelle on diminue d'une unite, la première variable, plus à la moitié de la même fonction dans laquelle on diminue la seconde variable, d'une unité. Cette égalité est une de ces équations que l'on nomme équations aux différences partielles.

On peut déterminer à son moyen, les probabilités de A, en partant des plus petits nombres, et en observant que la probubilité ou la fonction qui l'exprime, est égale à l'unité, lorsqu'il ne manque aucun point au joueur $A$, ou lorsque la première variable est nulle; ct que cette fonction devient nulle avec la scconde variable. En supposant ainsi quil ne manque qu'un point au joueur $A$, on trouve que sa probabilité est $\frac{1}{2}, \frac{3}{4}, \frac{7}{8}$, etc., suivart qu'il manque à $B$, un point, ou deux, ou trois, etc. Généralement, elle est alors égale à l'unité, moins $\frac{1}{2}$ élevé à une puissance égale 
au nombre des points qui manquent à B. Ora supposera ensuite qu'il manque deux points au joueur $\mathbf{A}$, et l'on trouvera sa probabilité égale à $\frac{1}{4}, \frac{1}{2}, \frac{1}{16}$, etc., suivant qu'il manque à B, un point, ou deux, ou trois, etc. On supposera encore qu'il manque trois points au joueur $A$, et ainsi de suite.

Cette manière d'obtenir les valeurs successires d'une quantité, au moyen de son équation aux différences, est longue et pénible; et les géomètres ont cherché des méthodes pour avoir la fonction générale des variables qui satisfait à cette équation, ensorte que l'on n'ait besoin pour chaque cas particulier, que de substituer dans cette fonction, les valesurs correspondantes des variables. Considérons cet objet d'une manière générale. Pour cela, concevons une suite de termes disposés sur une ligne horizontale, et tels que chacun d'eux dérive des précédens, suivant une loi donnée : supposons cetle loi expritnée par une équation entre plusjeurs termes consécutifs, et leur indice, ou le nombre qui indique le rang qu'ils occupent dans la série : cette équation est ce que je nomme équation aux différences finies à un seul indice variable. L'ordre ou le degré de celte équation est la différence du rang de ses dcux termes extrêmes. On peut, à son moyen, déterminer 


\section{SUR LES PROBABILITÉS.}

successivement les termes de la série, et la continuer indéfiniment; mais il faut pour cela, connaître un nombre de termes de la série, égal au degré de l'équation. Ces termes sont les constantes arbitraires de l'expression du terme général de la série, ou de l'intégrale de l'équation aux différences.

Concevons maintenant, au-dessus des termes de la série précédente, une seconde série de termes disposés horizontalement; concevons encore, au-dessus des termes de la seconde série, une troisième série horizontale, et ainsi de suite à l'infini, et supposons les termes de toutes ces sćries, liés par une équation générale cntre plusieurs termes consécutifs, pris tant dans le sens horizontal, que dans le sens vertical, et les nombres qui indiquent leur rang dans les deux sens. Cette équation est ce que je nomme équation aux différences finies partielles à deux indices variables.

Concevons pareiilement au-dessus du plan qui renferme les séries précédentes, un second plan renfermant des séries semblables, dont les termes soient placés respectivement au-dessus de ceux que contient le premier plan. Concevons ensuite au-dessus de ce second plan, un troisième plan renfermant des sérits semblables, et ainsi à l'infini.Supposons 
tous les termes de ces séries, liés par un équation entre plusieurs termes consécutifs, pris tant dans le sens de la longueur, que dans les sens de la largeur et de la profondeur, et les trois nombres qui indiquent leur rang dans ces trois sens. Cette équation est ce que je nomme équation aux différences finies partielles a trois indices variables.

Enfin, en considérant la chose d'une manière abstraite et indépendante des dimensions de l'espace, concevons généralement un système de grandeurs qui soient fonctions d'un nombre quelconque d'indices variables, et supposons entre ces grandeurs, leurs différences relatives à ces indices et les indices eux-mêmes, autant d'équations qu'il y a de ces grandeurs; ces équations scront aux différences finies partielles à un nombre quelconque d'indices variables.

On peut à leur moyen, déterminer successivement ces grandeurs. Mais de même que l'équation à un seul indice, exige que l'on connaisse un certain nombre de termes de la série; de même l'équation à deux indices exige que l'on connaisse une ou plusieurs lignes de sérics, dont les termes généraux peuvent chacun être exprizés par une fonction arbitraire d'un des indices. Parcillement, l'équation à trois indices exige que l'on connaisse un on 
plusieurs plans de séries, dont les termes généraux peuvent être exprimés chacun par une fonction arbitraire de deux indices, et ainsi de suite. Dans tous ces cas, on pourra, par des éliminations successives, déterminer un terme quelconque des séries. Mais toutes les équations entre lesquelles on élimine, étant comprises dans un même système d'équations générales; toutes les expressions des termes successifs que l'on obtient par ces éliminations, doivent être comprises dans une expression générale, fonction des indices qui déterminent le rang du terme. Cette expression est l'intégrale de l'équation proposée aux différences, et sa recherche cst l'objet du calcul intégral. Parmi les méthodes imaginées pour y parvenir, celle qui me paraît être la plus générale ct la plus simple, est fondée sur la considération des fonctions génératrices dont voici lidée.

Si l'on conçoit une fonction $\Lambda$ d'une variable, développée dans une série ascendante par rapport aux puissances de cette variable; le coeflicient de l'une quelconque de ces puissances sera fonction de l'indice ou exposant de cette puissance. A est ce que je nomme fonction génératrice de ce coefficient, ou de la fonction de l'indice.

Maintenant, si l'on multiplie la série $\mathbf{\Lambda}$, par 
une fonction linéaire de la variable, telle, par exemple, que l'unité plus deux fois cette variable; le produit sera une nouvelle fonction génératrice dans laquelle le coefficient d'une puissance quelconque de la variable, sera égal au coefficient de la même puissance dans $A$, plus au double du coefficient de la puissance inférieure d'une unité. Ainsi la fonction de l'indice dans le produit, égalera la fonction de l'indice dans A, plus le double de cette même fonction dans laquelle l'indice est diminué de l'unité. Cette fonction de l'indice dans le développement du produit, peut ainsi être envisagée, comme une dérivée de la function de l'indice dans $\mathbf{A}$, dérivée que l'on peut exprimer par une caractéristique piacée devant cette dernière fonction. La dérivation indiquée par la caractéristique, dépend de la fonction multiplicateur, que nous désignerons généralement par B, et que nous supposerons développée comme $\mathbf{A}$, par rapport aux puissances de la variable.

Si l'on multiplie de nouveau par B, le produit de $\mathbf{A}$ par $\mathrm{B}$, ce qui revient à multiplier $\mathbf{A}$ par le carré de B; on formera une troisième fonction génératrice dans laquelle le coefficient d'une puissance quelconque de la variable, sera une dérivée semblable du coefficient cor. respondant dans le premier produit; on pourra 
donc l'exprimer par la même caractéristıque placée devant la dérivée précédente, et alors cette caractéristique sera deux fois écrite devant le coefficient correspondant dans la série A; mais au lieu de l'écrire ainsi deux fois, on lui donne pour exposant, le nombre deux.

En continuant de cette manière, on voit généralement que si lon multiplie $A$ par une puissance $n^{i e m e}$ de $\mathrm{B}$; on aura le coefficient d'une puissance quelconque de la variable dans le produit, en plaçant devant le coefficient correspondant de $\mathrm{A}$, la caractéristique avec $n$ pour exposant. .

Supposons que $B$ soit l'unité divisée par la variable; alors dans le produit de $\mathbf{A}$ par $\mathbf{B}$, le coefficient d'une puissance de la variable, sera le coefticient de la puissance supérieure d'une unité dans $\boldsymbol{A}$; d'où il suit que dans le produit de $\mathbf{A}$ par la puissance $n^{i c m e}$ de $\mathbf{B}$, ce coefficient sera celui dela puissance supérieure d'un nombre $n$ d'unités dans $A$.

Si B est égal à, moins un plus l'unité divisée par la variable; alors dans le produit de A par $B$, le coefficient de la variable sera le coefficient de la puissance supérieure d'une unité dans A, moins le coefficient de cette puissance; il sera donc la différence finie de ce dernier cuefficient dans lequel on fait varier l'indice, de l'unité. Ainsi dans le prąduit de A 
par la puissance $n^{\text {ième }}$ de $\mathrm{B}$, le coefficient scra la différence $n^{i e ̀ m e}$ du coefficient correspondant dans A.

B étant une fonction de la variable, et $\mathbf{C}$ étant une autre fonction de la même variable; on pourra considérer $B$, comme une fonction de $\mathbf{C}$, développée dans une série ordonnée par rapport aux puissances de $\mathrm{C}$; le produit de A par cette série, sera donc identiquement égal au produit de A par B; et les coefficiens d’une même puissance de la variable, seront identiquement égaux dans ces deux produits. Máis le premier de ces coefficiens est formé d'une suite de termes correspondans aux produits de A par les diverses puissances de $\mathbf{C}$. Dans le produit de $\mathrm{A}$ par $\mathrm{C}$, ce coefficient est une nouvelle dérivée du coefficient correspondant dans $A$, dérivée que nous exprimerons par une nouvelle caractéristique placée devant ce dernjer coefficient. En changeant donc les diverses puissances de $\mathrm{C}$, dans cette nouvelle caractéristique affectée d'exposans égaux à ceux de ces puissances, et placée devant le coefficient correspondant de $\mathrm{A}$; en multipliant ensuite par ce coefficient, le terme indépendant de $\mathbf{C}$, dans la série précédente; on aura le coefficient relatif au produit de $\mathbf{A}$ par le développement de $B$, suivant les puissances de C. Si l'on égale ce coefficient, à celui 
SUR LES PROBABLLTTES.

gुui est relatif au produit de $\mathrm{A}$ par $\mathrm{B}$, et qui est exprimé par la première caractéristique placéc devant le coeflicient correspondant de A; on aura l'expression de la dérivée indiquée pur cette caractéristique, dans une séric ordonnée suivant les exposans de la nouvelle caractéristique. On voit que pour former cette série, c'est-à-dire pour repasser des fonctions génératrices à leurs coefficiens, il suffit de substituer dans B considéré comme fonction de $\mathrm{C}$, la nouvelle caractéristique, à la place de $\mathrm{C}$; de dívelopper cnsuite $\mathrm{B}$, dans une série ordonnée par rapport aux puissances de cette caractéristique; enfin d'écrire le coefficient d'une puissance indéterminée de la variable dans $A$, à la suite de chaque puissance de la caractéristique, et après le premier terme de la séric. Ainsi ce coefficient étant une fonction quelconque de l'indice de la puissance de la variable; la transformation d'une dérivée de cette fonction, indiquée par une première caractéristique, dans une série ordonnée par rapport aux exposans successifs de la caractéristique d'une nouvelle dérivée de la même fonction, se réduit aux opérations algébriques du développement des fonctions en séries.

Si l'on suppose B égal à l'unité divisée par la variable, et $\mathrm{C}$ égal a cette fraction moins un; $\mathrm{B}$ sera égal à l'unité plus $\mathrm{C}$, et le produit 
de A par la $n^{i e ̀ m e}$ puissance de B, sera égal aud produit de A par le développement de la puissance $n^{\text {ieme }}$ du binome, un plus $\mathrm{C}$; or le coefficient d'une puissance quelconque de la variable, dans le produit de A par B élevé à la $n^{i \grave{m} m e}$ puissance, est, comme on l'a vu, le coefficient de la puissance supérieure de $n$ unités, dans $\mathrm{A}$; et ce même coefficient dans le produit de A par une puissance de $C$, est la différence du même ordre, du coefficient correspondant dans $\Lambda$; une fonction quelcongue de l'indice augmenté de $n$, est donc égale aux coefficiens des termes du développement de la puissance $n^{i e m e}$ du binome, multipliés respectivement par la fonction elle-même, et ses différences successives; ce qui donne l'interpolation des séries, au moyen des différences de leurs termes successifs.

B étant tocijours supposé égalà l'unité divisće par la variable, et $\mathrm{C}$ étant une fonction quelconque de cette variable; $\mathrm{C}$ sera la même fonction du quotient de l'unité divisée par B. Si de là on tire l'expression de la puissance $n^{i \grave{m} e}$ de $\mathrm{B}$, dans une série développée suivant les puissances de $\mathbf{C}$; on aura en repussant des fonctions génératrices aux coefficiens, une fonction quelconque de l'indice augmenté de $n$, égale à une série dont le premier terme sera le premier terme de la série précédente, 


\section{SUR LES PROBABILITÉS.}

multiplié par la fonction elle-même; et dont les suivans seront ceux de la même série, dans lesquels, au lieu des puissances de C, on écrit les mêmes puissances de la caractéristique relative à C, suivies de la fonction. Si l'on suppose un des termes de cette nouvelle série, égal à zéro; tous les termes suivans seront nuls, et la somme des termes précédens sera l'expression de la fonction de l'indice augmenté de l'indéterminée $n$; cette expression sera l'intégrale complète de l'équation aux différences, indiquée par l'égalité du terme de la série, à zéro; on a ainsi la méthode la plus simple d'intégrer ce genre d'équations.

Concevons présentement que A soit une fonction de deux variables, ( ce que nous allons dire, s'étend à un nombre quelconque de variables). En la développant dans une série ordonnée par rapport aux puissances de ces variables, et à leurs produits; le coefficient du produit de deux puissances quelconques dans ce développement, sera une fonction des indices de ces puissances, dont A sera la fonction génératrice.

Si l'on multiplie A par une autre fonction $B$ de ces deux variables; le coefficient des deux mêmes puissances dans le produit, sera une fonction dérivée du coefficient précédent, dérivée que l'on pourra exprimer par une 
caractéristique placée devant ce coefficient. On verra, comme ci-dessus, que le coefficient correspondant, dans le produit de A par une puissance quelconque de $B$, sera exprimé par cette caractéristique, toujours placée devant le coefficient relatịf à $A$, et à laquelle on donne pour exposant, celui de la puissance de B. De lì résultent des théorèmes analogues à ceux qui sontrelatifs à une seule variable. On pourra développer d'une manière semblable, une fonction quelconque des deux indices augmentés respectivement des nombres $n$ el $n^{\prime}$, dans unc série ordonnée par rapport aux puissances d'une caractéristique, placées devant la fonction sans accroissement d'indices; et dont le premicr terme est cette fonclion elle-rnême. Si l'un des termes de cette série, est égal à zéro; tous les termes suivans le seront pareillement, et la somme des termes précédens sera l'expression de la fonction des deux indices augmentés respectivement des indéterminées $n$ et $n^{\prime}$ : cette expression sera l'intégrale de l'équation aux différences finies partielles, donnée par cette égalité.

Il existe toujours une fonction des variables, telle qu'cn la développant en série, les coefficiens des produits de leurs puissances ont entre cux, la relation donnce par une ćquation aux différences partielles. Cette fonction que 


\section{SUR LES PROBABILTTES.}

j’ai nomméc fonction génératrice de l'équation proposée, est souvent facile à obtenir : toutes les manières de la développer en série, donneront l'integrale de cette équation, sous des formes diverses plus ou moins commodes selon les circonstances.

Si l'on a une série ordonnée par rapport aux puissances d'une variable, et telle que le coeflicient de chaque puissance soit, par exemple, la moitié du coefficient de la puissance précédente; on pourra concevoir l'intervalle des deux premiers termes, rempli d'une infinité de termes dans lesquels les puissances de la variable croîtront par degrés infiniment petils, depuis zéro jusqu'à l'unité, et auront des coefficiens arbitraires. Les intervalles des termes consćcutifs suivans, seront parcillement remplis d'une infinité d'autres termes, mais dépendans des premiers, de manière que le cocficient d'une puissance de la variable, soit la moitié du coefficient de la puissance moindre d'une unité. Le plus communément, on suppose les intervalles des premiers termes de chaque série, remplis par des ordonnées paraboliques; alors les autres intervalles sont remplis d'ordonnées semblables, liées aux précédentes, par la loi générale de la sériequirenferme ainsi toutes les puissances entières et fractionnaires de la variable. . 
Supposons maintenant que $A$ soit une série semblable, et que B soit égal à, moins un plus l'unité divisée par une puissance $i$ entière ou fractionnaire de la variable. En représentant par un plus C, l'unité divisée par la variable; B sera égal à la quantité suivante, moins un plus la puissance $i$ du binome, un plus C. Si l'on multiplie par A, la puissance $n^{i e ̀ n e}$ de cette quantité; on aura un produit identiquement égal à celui de A par la puissance $n^{i e m e}$ de B. Sil'on développe ces puissances; on repassera des fonctions génératrices, aux coefliciens, $I^{\circ}$ en changeant la puisșance $n^{i \grave{m} e}$ de $\mathrm{B}, \mathrm{mul}$ tipliée par $\mathbf{A}$, dans la différence $n^{i e ̀ n e}$ de la fonction de l'indice, relative à $A$, $i$ étant l'accroisscment de l'indice; $2^{\circ}$ en changeant parcillement le produit de A par une puissance de C d'un ordre quelconque, dans une difference du même ordre, de la même fonction de l'indice, l'unité étant l'accroissement de l'indice. On aura donc la différ ence $n^{\text {ième }}$ d'une fonction quelconque de l'indice dont $i$ est l'accroissement, exprimée par une série des différences de la même fonction, dans lesquelles l'unité est l'accroissement de l'indice. On peut. ainsi transformer la caractéristique relative à un accroissement de l'indice, dans une série de caractéristiques relatives à un autre acoroissement. 
On voit dans tout ce qui précède, que les opérations algébriques relatives aux transformations des fonctions, se transportent aux caractéristiques, en leur donnant pour exposans, ceux des quantités qui leur correspondent. Cette analogie remarquable et féconde des puissances et des caractéristiques, avait été aperçue par Leibnitz dans les expressions différ entielles. Lagrange, en suivant cet aperçu de Leibnitz dans tous ses développemens, en a tiré des formules aussi curieuses qu'utiles pour l'analyse, mais sans en donner les démonstrations qu'il regardait comme difficiles. La théorie des fonctions génératrices ne laisse rien à desirer à cet égard, et de plus elle étend à des caractéristiques quelconques, l'analogie que ces deux grands géomètres n'avaient observée que relativement aux puissances et aux différences.

Si l'on suppose les accroissemens des indices, infiniment petits; les résultats relatifs à leurs accroissemens finis, subsisteront toujours, et se simplifieront en rejetant les infiniment petits d'un ordre supérieur à celui que l'on conserve. Ces passages du fini à l'infiniment petit, ont l'avantage d'éclairer les points délicats de l'analyse infinitésimale, qui ont été l'objet de grandes discussions parmi les géomètres. C'est ainsi que j’ai démontré 
la possibilité d'introduire des fonctions diotcontinues, dans les intégrales des équations aux différentielles partielles; pourvu que la discontinuité n'ait lieu que pour les difærentielles des fonctions, de l'ordre de ces équations. Les résultats transcendans du calcul sont, comme toutes les abstractions de l'entendement, des signes généraux dont on ne peut connaître la véritable étendue, qu'en remontant par l'analyse métaphysique, aux idées élémentaires qui y ont conduit; ce qui présente souvent de grandes difficultés; car l'esprit humain en éprouve moins encore à se porter en avant, qu'à se replier sur luimême.

Le passage du fini à l'infiniment pelit, répand un grand jour sur la métaphysique du calcul différentiel. On voit clairement par ce passage, que ce calcul n'est que la comparaison des coefficiens des mêmes puissances des différenticlles, dans le développement en série, de fonctions identiquement égales des indices augmentés respectivement de différentielles indéterminées. Les quantités que l'on néglige comme étant d'un ordre d'infiniment petits, supérieur à celui que l'on conserve, et qui semblent par cette, omission, ôter à ce calcul la rigueur de l'algèbre, ne sont que des puissances de ces différentielles, d'un ordre 


\section{SUR LES PROBABILITĹS.}

supérieur à celui des puissances dont on conpare les coefficiens, et qui par lì, doivent être rejelćes de cette comparaison; ensorte que le calcul différentiel a toute l'exactitude des autres opérations algébriques. Mais dans ses applications à la géométrie et à la mécanique, il est indispcnsable d'introduire le principe des limites. Par exemple, la soutangente d'une courbe étant la limite géométrique de la sousécante, ou la ligne dont celle-ci approche sans cesse, a mesure que les points d'intersection de la sćcante et de la courbe se rapprochent; l'expression analytique de la soutangente, doit être pareillement la limite de l'expression analytique de la sousćcante; clle est, par conséquent, égale at premier termo de cette dernicre expression développéc suivant les puissances de lintervaille qui sépare les deux points d'intersection.

On peat encore cnvisager la tangente, comme la droite dont léquation approche le plus de celle de la courbe, près du point de contingence. L'ordonnée de celte courbe, étant une fonction de l'abscisse; si à partir de ce point, on fait croitre l'abscisse, d'une quantité indétcrminée, et qu’on développe la fonction suivant les puissances de cette indéterminée; il est visible que la somme des deux premicrs termes de ce développement, 
sera l'ordonnée de la droite la plus approchante de la courbe; conséquemment, elle sera l'ordonnée de la tangente : le coefficient de l'indéterminée dans le second terme, exprimera le rapport de l'ordonnée à la soutangente. Il est facile de prouver par le principe des limites, que toute autre droite menée par le point de contingence, entrerait dans la courbe près de ce point.

Cette manière singulièrement heureuse de parvenir à l'expression des soutangentes, est due à Fermat qui l'a étendne aux courbes transcendantes. Ce grand géomètre exprime par la caractéristique $\mathbf{E}$, l'accroissement de l'abscisse; et en ne considérant que la première puissance de cet accroissement, il détermine exactement comme on le fait par le calcul différentiel, les soutangentes des courbes, leurs points d'inflexion, les maxima et minima de leurs ordonnées, et génćralement ceux des fonctions rationnelles. On yoit même par sá belle solution du problème de la réfraction de la lumière, en supposant qu'elle parvient d'un point ì un autre dans le temps le plus court, et qu'elle se meut dans les divers milieux diaphanes avec différentes vitesses, on voit dis-je, qu'il savait étendre sa méthode, aux fonctions irrationnelles, en se débarrassant des irrationnalités, par 
lélévation des radicaux aux puissances. On doit doncregarder Fermat, comme le véritable inventeur du calcul différentiel. Newton a depuis rendu ce calicul, plus analytique, dans sa Méthode des Fluxions; etil en a simplifié et généralisé les procédés, par son beau théorème du binome. Eufin presqu'en même temps, Leibnitz a enrichi le calcul différentiel, d'une notation qui en indiquant le passage du fini à l'infiniment petit, réunit à l'avantage d'exprimer les résultats rigoureux de ce calcul, celui de donner les premières valeurs approchécs des différences et des sommes des quantités; notation qui s'est adaptée d'elle-même au calcul des diférentielles partielles. La langue de l'analyse, la plus parfaite de toutes les langues, étant par elle-mếme un puissant instrument de découvertes; ses nolations, lorsqu'elles sont nécessaires et heureusement imaginées, sont des germes de nouveaux calculs. Ainsi, la simple idée qu'cut Descartes, d'indiquer les puissancesreprésentées pardeslettres, enécrivant vers le haut de ces lettres, les nombres qui expriment les degrés de ces puissances, a donné naissance au calcul exponentiel; et Leibnitz a été conduit par sa notation, à l'analogie singulière des puissances et des différentielles. Le calcul des fonctions génératrices, qui, comme onl'a vu, donnela véritable origine. 
de ceitte analogie, offre tant d'exemples de ce transport des exposans des puissances aux caractéristiques, qu'il peut encore être envisagé comme le calcul exponentiel des caractéristiques.

On est souvent conduit à des expressions qui contiennent tant de termes et de facteurs, que les substitutions numériques y sont impraticables. C'est ce qui a lieu dans les questions de probabilité, lorsque l'on considère un grand nombre d'événemens. Cependant il importe alors d'avoir la valeur numérique des formules, pour connaitre avec quelle probabilité, les résultals que les événemens développent en se multipliant, sont indiqués. Il importe surtout d'avoir la loi suivant laquelle cette probabilité approche sans cesse de la certitude qu'elle finirait par atteindre, si le nombre des événemens devenait infini. Pour y parvenir , je considérai que les intégrales définies de différentielles multipliées par des facteursélevésà de grandespuissances, donnaient par l'intégration, des formules composćes d'un grand nombre de termes et de facteurs. Cette remarque me fit naître l'idée de transformer dans de semblables intégrales, les expressions compliquées de l'analyse et les intégrales des équations aux différences. Je remplis cet objet par une méthode qui donne à-la-fois, la 
fonction comprise sous le signe intégral, et les limites de l'intégration. Elle offre cela de remarquable, suvoir, que cette fonction est la fonction même génératrice des expressions et des équations proposées; ce qui raltache cette méthode, à la théorie des fonctions génératrices, dont elle est le complément. Il ne s'agissait plus ensuite que de réduire l'intégrale définie, en série convergente. C'est ce que j'obtins par un procédé qui fait converger la série, avec d'autant plus de rapidité, que la formule qu'elle représente, est pluscompliquće; ensorte qu'il est d'autant plus exact, qu'il devient plus nécessaire. Le plus souvent, la série a pour facteur, la racine carrée du rapport de la circonférence au diamètre : quelquefois elle dépend d'autres transcendantes dont le nombre est infini.

Une remarque importante, qui tient à la grande généralité de l'analyse, et qui permet d'étendre cette méthode, aux formules et aux équations aux différences, que la théorie des probabilités présente le plus fréquemment, est que les séries auxquelles on parvient, en supposant réelles et positives, les limites des intégrales définies, ont également lieu dans le cas ou l'équation qui détermine ces limites, n'a que des racines négatives ou imaginaires. Ces passages du positif au négatif, et du rées 
à l'imaginaire, dont j'ai fait le premier usage, m'ont conduit encore aux valeurs de plusieurs intégrales définies singulières, que j'ai trouvées ensuite directement. On peut donc considérer ces passages, comme des moyens de découvertes, pareils à l'induction et à l'analogie employées depuis long-temps par les géomètres, d'abord avec un extrême réserve, ensuite avec une entière confiance; un grand nombre d'exemples en ayant justifié l'emploi. Cependant il est toujours utile de confirmer par des démonstrations directes, les résultats obtenus par ces civers moyens.

J'ai nommé calcul des fonctions généra. trices, l'ensemble des méthodes précédentes: ce calcul sert de fondement à la théorie des probabilités, exposée dans cet ouvrage.

APPLICATIONS DU CALCUL DES PROBABILITESS.

Des Jeux.

Les combinaisons que les jeux présentent, ont été l'objet des premières recherches sur les probabilités. Dans l'infinie variété de ces combinaisons, plusieurs d'entre ellesse prêtent avec facilité au calcul : d'autres exigent des calculs plus difficiles; et les difficultés croissanti mesure queles combinaisons deviennent 
plus compliquées, le desir de les surmonter et la curiosité ont excité les géomètres à perfectionner de plus en plus, ce genre d'analyse. On a vu précédemment que l'on pouvait facilement déterminer par la théorie des combinaisons, les bénéfices d'une loterie. Mais il est plus difficile de savoir en combien de tirages on peut parier un contre un, par exemple, que tous les numéros seront sortis. $n$ étant le nombre des numéros, $r$ celui des numéros sortans à chaque tirage, et $i$ le nombre inconnu de tirages; l'expression de la probabilité de la sortie de tous les numéros, dépend de la différence finie $n^{i e m e}$ de la puissance $i$ du produit de $r$ nombres consécutifs. Lorsque le nombre $n$ est considérable, la recherche de la valeur de $i$, qui rend cette probabilité égale à $\frac{x}{2}$, devient impossible, à moins qu'on ne convertisse cette différence, dans une série très-convergente. C'est ce que l'on fait heureusement par la méthode ci-dessus indiquée, pour les approximations des fonctiøns detrèsgrands nombres. On trouve ainsi que la loterie étant composée de dix mille numéros dont un seul sort à chaque tirage; il y a du désavantage à parier un contre un, que tous les numéros sortiront dans $9^{5} 767$ tirages, et de l'avantage à faire le même pari pour $9^{5} 768$ tirages. $\Lambda$ la loterie de France, ce pari est 
désavantageux pour 85 tirages, et avantageux pour 86 tirages.

Considérons encore deux joueurs $A$ et $B$ jouant ensemble a croix et pile, de manière qu'à chaque coup, si croix arrive, A donne un jeton a $B$ qui lui en donne un, si pile arrive; le nombre des jetons de $B$ est limité: celui des jetons de A est illimité; ct la partie ne doit finir que lorsque B n'aura plus de jetons. On demande en combien de coups, on peut parier un contre un, que la partie sera terminée. L'expression de la probabilité que la partie sera terminée dans un nombre $i$ de coups, est donnée par une suite qui renferme un grand nombre de termes et de facteurs, si le nombre des jetons de $\mathbf{B}$ est considérable; la recherche de la valeur de l'inconnue $i$ qui rend cette suite égale $\dot{a} \frac{1}{2}$, serait donc alors impossible, si l'on ne parvenait pas à réduire la suite dans une série très-convergente. En lui appliquant la méthode dont on vient de parler, on trouve une expression fort simple de l'inconnue, de laquelle il résulte que si, par exemple, B a cent jetons; il y a un peu moins d'un contre un à parier que la partie sera finie en 23780 coups, et un peu plus d'un contre un à parier qu'elle sera finie dans 23781 coups.

Ces deux exemples joints à ceux que nous 
SUR LES PROBABILITÉS.

avons déjà donnés, suffisent pour faire voir comment les problèmes sur les jeux ont pu contribuer à la perfeclion de l'analyse.

Des inégalités inconnues quipeuvent exister entre les chances que l'on supposégoles.

Les inégalités de ce genre ont sur les résultats du calcul des probabilités, une influence sensible qui mérite une attention particulière. Considérons le jeu de croix et pile, et supposons qu'il soit également facile d'amener l'une ou l'autre face de la pièce. Alors la probabilité d'amener croix au premier coup est $\frac{1}{2}$, et celle de l'amener deux fois de suite, est $\frac{1}{4}$. Mais s'il existe dans la pièce, une inégalité qui fasse paraître une des faces plutôt que l'autre, sans que l'on connaisse quelle est la face favorisée par cette inégalité ; la probabilité d'amener croix au premier coup sera toujours $\frac{1}{2}$; parce que dans l'ignorance ou l'on est de la face que cette inégalité favorise, autant la probubilité de l'événement simple est augmentée, si cette inégalité lui est fuvorable, autant elle est diminuée, si l'inégalité lui est contraire. Mais dans cette ignorance même, la probabilité d'amener croix deux fois de suite, est augmentée. En effet, cette probabilité est celle d'amener croix au premier 
coup, multipliée par la probabilité que l'ayant amené au premier coup, on l'amènera au second; or son arrivée au premier coup est un motif de croire que l'inégalité de la pièce le favorise; l'inégalité inconnue augmente donc alors la probabilité d'amener croix au second coup; elle accroit par conséquent le produit des deux probabilités. Pour soumettre cet objet au calcul, supposons que cette inégalité augmente d'un vingtième, la probabilité de l'événement simple qu'elle favorise. Si cet événement est croix, sa probabilité sera $\frac{t}{x}$ plus $\frac{1}{20}$ ou $\frac{15}{90}$, et la probabilité de l'amener deux fois de suite, sera le carré de $\frac{11}{20}$ ou $\frac{121}{400}$. Si l'événement favorisé est pile, la probabilité de croix sera $\frac{1}{2}$ moins $\frac{1}{20}$ ou $\frac{9}{20}$, et la probabilité de l'amener deux fois de suite sera $\frac{8 \mathrm{r}}{400}$. Comme on n'a d'avance, aucune raison de croire que linégalité fivorise l'un de ces événemens plutôt que l'autre; il est clair que pour avoir la probabilité de l'événement composé croix croix, il faut ajouter les deux probabilités précédentes, et prendre la moiitié de leur somme; ce qui donne $\frac{\text { rot }}{400}$ pour cette probabilité qui surpasse $\frac{1}{4}$ de $\frac{1}{400}$, ou du carré de l'accroissement $\frac{x}{20}$ que linégalité ajoute à la possibilité de l'événement qu'elle favorise. La probabilité d'amener pile pite est parcillement $\frac{101}{40}$; mais les probabilités d'amener croix 


\section{SUR LES PROBABILITES.}

pile ou pile croix ne sont chacune, que $\frac{99}{40 ;}$; car la somme de ces quatre probabilites, doit égaler la certitude ou l'unité. On trouve ainsi généralcment que les causes constantes et inconnues qui favorisent les événemens simples que l'on juge également possibles, accroissent toujours la probabilité de la répétition d'un même événement simple.

Dans un nombre pair de coups, croix et pile doivent arriver tous deux, ou un nombre pair ou un nombre impair de fois. La probubilité de chacun de ces cas est $\frac{1}{2}$, si les possilialités des deux fuces sont égales; mais s'il existe entre elles, une inégalité inconnue, cette inégalité est toujours fuvorable au premier cas.

Deux joueurs dont on suppose les adresses égales, jouent avec les conditions qu'à chaque coup, celui qui perd, donne un jeton à son adversaire, et que la partie dure, jusqu'à ce que l'un des joueurs n'ait plus de jetons. Le calcul des probabilités nous montre que pour l'égalité du jeu, les mises des joueurs doivent être en raison inverse de leurs jetons. Mais s'il existe entre leurs adresses, une petite inégalité inconnue; elle favorise celui des joueurs qui a le plus pelit nombre de jetons. Sa probabilité de gagner la partie augmente, si les joueurs conviennent de dubbler, de tripler 
leurs jetons; et elle devient $\frac{1}{2}$ ou la même que la probabilité de l'autre joueur, dans le cas où les nombres de leurs jetons deviendraient infinis, en conservant toujours le même rapport.

On peut corriger l'influence de ces inégalités inconnues, en les soumettant elles-mêmes aux chances du hasarl. Ainsi au jeu de croix et pile, si l'on a une seconde piece que l'on projette chaque fois avec la premiere; et que l'onconvienne denommerconstamment croix, la face amenée par cette seconde pićce ; la probabilité d'amener croix deux fois de suite, avec la première pièce, approchera beaucoup plus d'un quart, que dans le cas d'une seule pièce. Dans ce dernier cas, la différence est le carré du petit accroissement de possibilité que l'inégalité inconnue donne à la face de la première pièce, qu'elle favorise : dans l'autre cas, cette différence est Ie quadruple produit de ce carré, par le carré correspondant relatif à la seconde pièce.

Que l'on jette dans une urne, cent numéros depuis un jusqu'à cent, dans l'ordre de la numération, et qu'après avoir agité l'urne, pour mêler ces numéros, on en tire un; il est clair que si le mélange a été bien fait, les probabilités de sortie des numéros, sont les mêmes. Mais si l'on craint qu'il n'y ait entre elles, de 
petites différences dépendantes de lordre suivant lequel les numéros ont été jetés dans l'urne; on diminucra considérablement ces différences, en jetant dans une seconde urne, ces numéros suivant leur ordre de sortie de la premiére urne, et en agitant ensuite cette seconde urne, pour mêler ces numéros. Une troisième urne, une quatricme, etc., diminueraient de plus en plus ces diflérences déjà insensibles dans la seconde urne.

\section{De la probabilité des témoignages.}

La plupart de nos jugcmens étant fondés sur la probabilité des témoignages, il est bien important de la soumettre au calcul. La chose, il est vrai, devient souvent impossible, par la difficulté d'apprécicr la véracité des témoins, et par le grand nombre de circonstances dont les faits qu'ils attestent, sont accompagnés. Miais on peut dans plusieurs cas, résoudre des problèmes qui ont beaucoup d'analogie avec les questions que l'on se propose, et dont les solutions peuvent être regardées comme des approximations propres à nous guider, et à nous garantir des erreurs et des dangers auxquels de mauvais raisonnemens nous exposent. Une approximation de ce genre, lorsqu'elle est bien dirigée, est toujours préférable 
aux raisonnemens les plus spécieux. Essayons donc de donner quelques règles génćrales pour y parvenir.

On a extrait un seul numéro, d'une urne qui en renferme mille. Un témoin de ce tirage, annonce que le $n^{\circ} 79$ est sorti; on demande la probabilité de cetle sorlie. Supposons que l'expérience ait fait connaitre que ce témoin trompe une fois sur dix, ensorle que la probabilité de son témoignage soil $\frac{9}{\text { ro. }}$ Ici, l'événement observé est le témoin attestant que le $n^{\circ} 79$ est sorti. Cet événement peut résulter des deux hypotheses suivantes, savoir, que le témoin énonce la vérité, ou qu’il trompc. Suivant le principe que nous avons exposé sur la probabilité des causes, tirée des événemens observés, il faut d'abord déterminer à priori, la probabilité de l'événement dans chaque hypothése. Dans la première, la probabilité que le témoin annoncera le $n^{\circ} 79$, est la probabilité même de la sortie de ce numéro, c’est-à-dire $\frac{1}{1000}$. Il faut la multiplier par la probabilité $\frac{9}{10}$ de la réracité du témoin; on aura donc $\frac{9}{1000}$ pour la probabilité de l'événement observé, dans celte hypothèse. Si le témoin trompe, le $n^{\circ} 79$ n'est pas sorti; et la probabilité de ce cas est $\frac{999}{1000}$. Mais pour annoncer la sortie de ce numéro, le témoin doit le choisir parmi les 999 numéros non sontis; ct comme il est 
supposé n'avoir aucun motif de préférence pour les uns plutòt que pour les autres, la probabilité qu'il choisira le $n^{\circ} 79$ est $\frac{1}{999}$; en multipliant donc cette probabilité, par la précédente, on aura $\frac{2}{1000}$ pour la probabilité que le témoin annoncera le $n^{\circ} 79$, dans la seconde liypothèse. Il faut encore multiplier cette probubilité, par la probabilité $\frac{1}{10}$ de l'hypothèse clle-même; ce cqui donne $\frac{1}{10000}$ pour la prohabilité de l'événement, relative à cetle hypothèse. Présentement, si l'on forme une fraction dont le numérateur soit la probabilité relative à la première hypothèse, et dont le dénominateur soit la somme des probabilités relatives aux deux hypothèses; on aura par le sixième prineipe, la probabilité de la première hypothèse, ct cette probabilité sera $\frac{9}{10}$, c'est-ì-dire la véracité même du témoin. C'est aussi la probabilité de la sorlie du n ${ }^{\circ}$ 79. La probabilité du mensonge du témoin et de la non-sortic de ce numéro est $\frac{1}{10}$.

Si le témoin voulant tromper, avait quelqu'iutcrêt à choisir le $n^{\circ} 79$ parmi les numéros non-sortis; 's'il jugeait, par exemple, qu'ayant placé sur ce numéro une mise considérable, l'annonce de sa sortie augmentera son crédit; la probabilité qu'il choisira ce numéro, ne sera plus, comme auparavant, $\frac{1}{999}$; elle pourra être alors $\frac{1}{2}, \frac{\pi}{3}$, etc., suivant l'intérêt qu'il aura 
d'annoncer sa sortie. En la supposant $\frac{1}{9}$, il faudra multiplier par cette fraction, la probabilité $\frac{999}{1000}$, pour avoir dans l'hypothèse du mensonge, la probabilité de l'événement observé, quil faut encore multiplier par $\frac{x}{10}$; ce qui donne $\frac{112}{10000}$ pour la probabilité de l'événement dans la seconde hypothèse. Alors la probabilité de la premiẻre hypothèse, ou de la sortie du n 79 , se réduit par la règle précédente, $\grave{a} \frac{9}{120}$. Elle est donc très-affaiblie par la considération de l'intérêt que le témoin peut avoir à annoncer la sortie du $n^{\circ} 79$. Le bon sens nous dicte que cet intérêt doit inspirer de la défiance. Majs le calcul en apprécie l'influence avec exactitude.

La probabilité à priori du numéro énoncé par le témoin, estl'unité divisée par le nombre des numéros de l'urne : elle se transforme en vertu du témoignage, dans la véracité même du témoin; elle peut donc être affaiblie par ce témoignage. Si, par exemple, l'urne ne renferme que deux numéros, ce qui donne $\frac{x}{2}$ pour la probabilité à priori de la sortie du $n^{\circ} 1$; et sil la véracité d'un témoin qui l'annonce est $\frac{4}{10}$; cette sortie en devient moins probable. En effet, il est visible que le témoin ayant alors plus de pente vers le mensonge que vers la vérité; son témoignage doit diminuer la probabilité du fait attesté, toutes les fois que 
cette probabilité égale ou surpasse $\frac{1}{2}$. Mais s'il y a trois numéros dans l'urne, la probabilitéé á priori de la sortie du $\mathrm{n}^{\circ} 1$, est accrue par l'affirmation d'un témoin dont la véracité surpasse $\frac{1}{3}$.

Supposons maintenant que l'urne renferme 999 boules noires et une boule blanche, et qu'une boule en ayant été extraite, un témoin du tirage annonce que cette boule est blanche. La probabilité de l'événement observé, déterminée à priori, dans la première hypothèse, sera ici, comme dans la question précédente, égale à $\frac{9}{\text { รоōo. }}$. Mais dans l'hypothèse ou le témoin trompe, la boulc blanche n'est pas sortic, et la probabilité de ce cas est $\frac{999}{1000}$. Il faut la mulliplier par la probabilité $\frac{1}{10}$ du mensonge, ce qui donne $\frac{999}{10000}$ pour la probabilité de l'événement observé, relative à la seconde hypothèse. Cette probabilité n'était que $\frac{1}{100 \overline{-0}}$ dans la question précédente : cette grande différence tient à ce qu'une boule noire étant sortie, le témoin voulant tromper, n'a point de chuix a faire parmi les 999 boules non sorties, pour annoncer la sortie d'une boule blanche. Maintenant, si l'on forme deux fractions dont les numérateurs soient les probabilités relatives à chaque hypothèse, et dont le dénominateur commun soit la somme de ces probabilités; on aura $\frac{9}{1.008}$ pour la probabilité 
de la premiere bypothí se, et de la sortie d'une boulc blanche, et $\frac{999}{1008}$ pour la probabilité de la seconde hypothese, et de la sortie d'une boule noire. Cette dernière probabilité est fort approchante de la certitude : elle en approcherait beaucoup plus encore, et deviendrait -999999 , si l'urne renfermait un million de boules dont une seule serait blanche; la sortie d'une boule blanche devenant alors beaucoup plus extraordinaire. On voit ainsi comment la probabilité du mensonge croît à mesure que le fait devient plus extraordinaire.

Nous avons supposé jusqu'ici que le témoin ne se trompait point; mais si l'on admet encore la chance de son erreur, le fait extraordinaire devient plus invraisemblable. Alors au lieu de deux hypothèses, on aura les quatre suivantes, savoir, celle du témoin ne trompant point et ue se trompant point; celle du témoin ne trompant point, et se trompant; ihypothc̀se du témoin trompant et ne se trompant point; enfin cclle du témoin trompant et se trompant. En déterminant à priori dans chacune de ces hypothèses, la probabilité de l'événement observé; on trouve par le sixième principe, la probabilité que le fait attesté est faux, égale à une fraction dont le numérateur est le nombre des boules noires de l'urne, multiplié par la somme des probabilités que 


\section{SUTR LES PROБABILTTÉS.}

le témoin ne trompe point et se trompe, ou qu'il irompe et ne se trompe point, et dont le dénominateur est ce numérateur augmenté de la somme des probabililés que le témoin ne trompe point et ne se trompe point, ou qu'il trompe et se trompe a -la-fois. On voit par lá, que si le nombre des boules noires de l'urne est très-grand, ce qui rend extraordinaire, la sortie de la boule blanche; la probabilité que le fait altesté n'est pas, approche extrêmement de la certitude.

En étendant cette conséquence, a tous les faits extraordinaires; il en résulte que la probabilité de l'errcur ou du mensonge du témoin, devient d'autant plus grandẹ, que le fait attesté est plus extraordinairc. Quelques auteurs ont avancé le contraire, en se fondant sur ce que la vue d'uu fait extraordinaire ctant parfaitement semblable à celle d'un fait ordinaire, les mêmes motifs doivent nous porter à croire également le témoin, soil qu'il aftirme l'un ou qu'il affirme l'autre de ces faits. Le simple bon sens repousse une aussi étrange assertion: mais le calcul des probabilités, en confirmant l'indication du sens commun, apprécie de plus, l'invraisemblance des témoignages sur les faits extraordinaires.

On insiste, et l'on suppose deux témoins cgalement dignes de foi, dont le premier 
atteste qu'il a vu mort, il y a quinze jours, un indivjdu que le second témoin affirme avoir vu hier, plein de vie. L'un ou l'autre de ces faits n'offre rien d'invraisemblable. La résurrection de lindividu est une conséquence de leur ensemble; mais les témoignages ne portant point directement sur elle, ce qu'elle a d'extraordinaire ne doit point affaiblir la croyance qui leur est due. (Encyclopédie, art. certi‡ude).

Cependant, si la conséquence qui résulte de l'ensemble des témoignages était impossible, l'un d'eux serait nécessairement faux; or une conséquence impossible est la limite des conséquences extraordinaires, comme l'erreur est la limite des invraisemblances; la valeur des témoignages, qui devient nulle dans le cas d'une conséquence impossible, doit donc être trèsaffaiblie dans celui d'une conséquence extraordinaire. C'est en effet, ce que le calcul des probabilités confirme.

Pour le faire voir, considérons deux urnes $A$ et $B$ dont la premiere contient un millio de boules blanches, et la seconde, un million de boules noires. On tire de l'une de ces urnes, une boule que l'on remet dans l'autre urne dont on extrait ensuite une boule. Deux témoins, l'un du premier tirage, et l'autre du second, attestent que la boule quils opt vu 


\section{SUR LES PROBABLLITÉS.}

extraire, cst blanche. Chaque témoignage pris isolément, n’a rien d'invraisemblable; et il est facile de voir que la probabilité du fait attesté, est la véracité même du témoin. Mais il suit de l'ensemble des témoignages, qu'une boule blanche a été extraite de l'urne $A$ au premier tirage, et qu'ensuite, mise dans l'urne B, elle a reparu au second tirage; ce qui est fort extraordinaire; car cette urne renfermant alors une boule blanche sur un million de boules noires, la probabilité d’en extraire la boule blanche est $\div \frac{1}{100001}$. Pour déterminer l'affaiblissement qui en résulte dans la probabilité de la chose énoncée par les deux témoins; nous remarquerons que l'événement observé est ici l'afirmation par chacun d'eux, que la boule quil a vu extraire, est blanche. Représentons par $\frac{9}{10}$ la probabilité qu'il énonce la vérité, ce qui peut avoir lieu dans le cas présent, lorsque le témoin ne trompe point et ne se trompe point, et lorsqu'il trompe et se trompe a-la-fois. On peut former les quatre hypothèses suivantes.

$1^{\circ}$. Le premier et le second témoin disent la vérité. Alors, une boule blanche a d'abord été extraite de l'urne $\mathbf{A}$, et la probabilité de cet événement est $\frac{1}{2}$, puisque la boule extraite au premier tirage a pu sortir également de l'une ou l'autre urne. Ensuite, la boule 
extraite mise dans l'urne $B$ a reparu au second tirage : la probabilité de cet événement est $\frac{\frac{1}{100001}}{200 \% \text { p }}$ la probabilité du fait énoncé est done 200000.2 En la multipliant par le produit des probabilités $\frac{9}{10}$ et $\frac{9}{10}$ que les témoins disent la vérité; on aura $\frac{81}{200000 \frac{0}{200}}$ pour la probabilité de l'événement observé, dans cetle première hypothèse.

$2^{\circ}$. Le premier témoin dit la vérité, et le second ne la dit point, soit qu'il trompe et ne se trompe point, soit qu'il ne trompe point et se trompe. Alors une boule blanche est sortie de l'urne $A$ au premier tirage, et la probabilité de cet événement est $\frac{1}{2}$. Ensuite cette boule ayant été mise dans l'urne $B$, une boule noire en a été extraite : la probabilité de cette extraction est $\frac{1000000}{10000001}$; on a donc $\frac{1000000}{2000002}$ pour la probabilité de l'événement composé. En la multipliant par le produit des deux probabilités $\frac{9}{10}$ et $\frac{1}{10}$ que le premier témoin dit la vérilé, et que le second ne la dit point; on aura $\frac{9000000}{200000000}$ pour la probábilité de l'événement observé, dans la seconde hypothèse.

$3^{\circ}$. Le premicr témoin ne dit pas la vérité, et le second l'énonce. Alors une boule noire est sortie de l'urne B au premier tirage, et après avoir été mise dons l'urne $\Lambda$, une bouls blanche a été extraite de celte urne. La probabilité du premier de ces événemens est $\frac{1}{2}$, 
et celle du second est $\frac{1000000}{2000001}$; la probabilitê de l'événement composé est donc $\frac{20000 \% 0}{2000000}$. En la multipliant par le produit des probabilités $\frac{1}{10}$ et $\frac{9}{10}$, que le premier témoin ne dit pas la vérilé, et que le second l'énonce; on aura $\frac{9000000}{200000 \frac{00}{100}}$ pour la probabilité de l'événement observé, relative à cette hypothèse.

$4^{\circ}$. Enfin, aucun des témoins ne dit la vórité. Alors une boule noire a été extraite de l'urne B au premier tirage; ensuite ayant été mise dans l'urne $A$, elle a reparu au second tirage : la probabilité de cet événement composé est $\frac{10000}{200}$. En la multipliant par le produit des probabilités $\frac{1}{10}$ et $\frac{3}{10}$ que chaque témoin ne dit pas la vérité; on aura $\frac{1}{200000200}$ pour la probabilité de l'événement observé, dans cette hypothèse.

Maintenant, pour avoir la probabilité de la chose énoncée par les deux témoins, savoir, qu'une boule blanche a ćté extraite à chacun des lirages; il faut diviser la probabilité correspondante ì la première hypothèse, par la somme des probabilités relatives aux quatre hypothèses; et alors on a pour cette probabilité $\frac{81}{18000082}$, fraction extrêmement petite.

Si les deux témoins affirmaient, le premier, qu'une boule blanche a ćté extraite de l'une des deux urnes $\mathbf{A}$ et $\mathbf{B}$; le second, qu'une boule blanche a ćté pareillement extraite de 
l'une des deux urnes $A^{\prime}$ et $B^{\prime}$, en tout senblables aux premières; la probabilité de la chose énoncée par les deux témoins serait le produit des probabilités de leurs témoignages ou $\frac{81}{100}$, c'est à-dire, cent quatre-vingt mille fois au moins, plus grande que la précédente. On voit par la, combien dans le premier cas, la réapparition au second tirage, de la boule blanche extraite au premier, conséquence extraordinaire des deux témoignages, en affitblit la valeur.

Nous n'ajouterions point foi au témoignage d'un homme qui nous attesterait qu'en projetant cent dés en l'air, ils sont tous retombés sur la même face. Si nous avions été nousmêmes spectateurs de cet événement, nous n'en croirions nos propres yeux, qu'après en avoir scrupuleusement examiné toutes les circonstánces, pour être bien sûrs qu'il n'y a point eu de prestige. Mais après cet examen, nous ne balancerions point à l'admettre, malgré son extrême invraisemblance; et personne ne serait tenté pour l'expliquer, de recourir à une illusion produite par un renversement des lois de la vision. Nous devoris en conclure que la probabilité de la constance des lois de la nature, cst pour nous, supérieure à celle que l'événement dont il s'agit, ne doit point avoir lieu; probabilité supérieure elle- 
même à celle de la plupart des faits historiques que nous regardons comme incontestables. On peut juger par lá, du poids immense de témoignages nécessaires pour admettre une suspension des lois naturelles; et combien il serait abusif d'appliquer à ce cas, les règles ordinaires de la critique. Tous ceux qui sans offrir cette immensité de témoignages, ćtayent ce quilis avancent, de récits d'événemens contraires à ces lois, affaiblissent plutôt qu'ils n'augmentent la croyance qu'ils cherchent à inspirer; car alors ces récits rendent très-probable, l'erreur ou le mensonge de leurs auteurs. Mais ce qui diminue la croyance des hommes éclairés, accroit souvent celle du vulgaire; ct nous en avons donné précédemment la raison.

11 y a des choses tellement extraordinaires, que rien ne peut en balancer l'invraisemblance. Mais celle-ci, par l'effet d'une opinion dominante, peut être affuiblic au point de paraitre inférieure à la probabilité des témoignages; et quand cette opinion vient à changer, un récit absurde admis unanimement dans le siècle qui lui a donné naissance, n'offre aux siècles suivans, qu'une nouvclle preuve de l'extrême influence de l'opinion générale, sur les meilleurs esprits. Deux grands hommes du siècle de Louis XIV, Racine et Pascal, en 
sont des exemples frappans. Il est amligeant de voir avec quelle complaisance, Racine, ce peintre admirable du coeur humain, et le poëte le plus parfait qui fut jamais, rapporte comme miraculeuse, la guérison de la jeune Perrier, nièce de Pascal, et pensionnaire à l'abbaye de Port-Royal : il est pénible de lire les raisonnemens par lesquels Piscal cherche à prouver que ce miracle devenait néccssaire à la religion, pour justifier la doctrine des religieuses de cette abbaye, alors persécutécs par les Jésuites. La jeune Perrier était depuis trois ans et demi, affligée d'une fistule lacrymale : elle toucha de son oeil malude, une relique que l'on prétendait être une des épines de la couronne du Sauveur, ct elle se crut à linstant, guérie. Quelques jours après, les médecins et les chirurgiens constaièrent la guérison, et ils jugèrent que la nature et les remèdes n'y avaient eu aueune part. Cet événement arrivé en 1656, ayant fait un grand bruit, « tout Paris se porta, dit Racine, à » Port-Royal. La foule croissait de jour en ») jour, et Dieu même semblait prendre plaisir „) à autoriser la dévotion des peuples, par la 》quantité de miracles qui se firent. en cette "église. " A cette époque, les miracles et les sortiléges ne paraissaient pas encore invraisemblables, et l'on n'hésitait point à leur 
attribuer les singularités de la nature, que l'on ne pouvait autrement expliquer.

Celte manière d'envisager les effets extraordinaires se retrouve dans les ouvrages les plus remarquables du siècle de Louis XIV, dans l'Essai même sur l'entendement humain, du sage Locke qui dit en parlant des degrés d'assentiment: « quoique la commune expé) rience et le cours ordinaire des choses aient

s) avec raison, une grande influence sur l'es) prit des lrommes, pour les porter à donner 3) ou à refuser leur consentement à une chose "qui leur est proposée à croire; il y a pour》) tant un cas où ce qu'il y a d'étrange dans 3) un fait, n'affaiblit point l'assentiment que ) nous devons donner au témoignage sincère »sur lequel il est fondé. Lorsque des événe》) mens surnaturels sont conformes aux fins 3) que se propose celui qui a le pouvoir de ") changer le cours de la nature, ils peuvent "être d'antant plus propres à trouver créance 》) dans nos esprils, quils sont plus au-dessus ) des observations ordinaires, ou même qu'ils 》y sont plus opposés. ) Les vrais principes de la probabilité des témoignages, ayant été ainsi méconnus des philosophes auxquels la raison est principalement redevable de ses progrès; j'ai cru devoir exposer avec étendue, les résultals du calcul sur cetimportant objet. 
Ici se présente naturellement la discussion d'un argument fameux de Pascal, que Craig, mathematicien anglais, a reproduit sous une forme géométrique. Des témoins attestent qu'ils tiennent de la Divinité même, qu'en se conformant ì telle chose, on jouira, non pas d'une, ou de deux, mais d'une infinité de vies heureuses. Quelque faible que soit la probabilité des témoignages, pourvu qu'clle ne soit pas infiniment petite, il est clair que l'avantage de ceux qui se conforment à la chose prescrite, est infini; puisqu'il est le produit de cette probabilité, par un bien infini; on ne doit donc point balancer à se procurer cet avantage.

Cet argument est fondé sur le nombre infini de vies heureuses promises au nom de la Divinité, par les témoins; il faudrait donc faire ce qu'ils prescrivent, précisément parce qu'ils exagèrent leurs promesses au-delit de loutes limites, conséquence qui répugne au bon sens. Aussi le calcul nous fait-il voir que celte exagération même affaiblit la probabilité de leur témoignage, au point de la rendre inliniment petite, ou nulle. En effet, ce cas revient à celui d'un témoin qui annoncerait la sortic du numéro le plus élevé, d'une urne remplie d'un grand nombre de numéros dont un seul a été extrait, et qui aurait un grand inlérêt 
it annoncer la sortie de ce numéro. On a vu précédernment combien cet intérêt affaiblit son témoignage. En n'évaluant qu'à $\frac{1}{2}$ la probabilité que si le témoin trompe, il choisira le plus grand numéro; le calcul donne la probabilité de son annonce, égale à une fraction dont le numérateur est le double de la probabilité de son témoignage, considérée $\dot{a}$ priori ou indépendamment de l'annonce, ct dont le dénominateur est le produit du nombre des numéros de l'urne, par l'unité diminuée de cette dernière probabilité. Pour assimiler ce cas, à celui de l'argument de Pascal; il suffit de représenter par les numíros de l'urne, tous les nombres possibles de vies heureuses, ce qui rend le nombre de ces numéros, infini; et d'observer que si les témoins trompent, ils ont le plus grand intérêt pour accréditer leur mensonge, à promettre une éternité de bonheur. L'expression précédente de la probabilité de leur témoignage, devient alors infiniment petite. En la multipliant par le nombre infini de vies heureuses promises, l'infini disparaît du produit qui exprime l'avantage résultant de cette promesse; ce qui détruit Pargument de Pascal.

Considérons présentement la probabilité de l'ensemble de plusicurs témoignages sur un fait déterminé. Pour fixer les idées, supposons 
que ce fait soit la sorlie d'un numéro d'une urne qui en renferme cent, et dont on a extrait un seul numéro. Deux témoins de ce tirage, annoncent que le $\mathrm{n}^{\circ} 1$ est sorti; et l'on demande la probabilité résultante de l'ensemble de ces témoignages. On peut former ces deux bypothèses: les témoins disent la vérité; les témoins trompent. Dans la premicre hypothèse, le $n^{\circ}$ I est sorti, et la probabilité de cet événement est $\frac{1}{100}$. Il faut la multiplier par le produit des véracités des témoins, véracilés que nous supposerons être $\frac{19}{10}$ et $\frac{7}{10}$; on aura done $\frac{63}{10000}$ pour la probabilité de l'événement observé, dans cette hypothése. Dans la scconde, le $n^{\circ}$ I n'est pas sorti, et la probabilité de cet événement est $\frac{99}{100}$. Mais l'accord des témoins exige alors qu'en cherchant a tromper, ils choisissent tous deux le numéro 1, sur les 99 numéros non sortis; la probabilité de ce choix est le produit de la fraction $\frac{1}{99}$ par elle-même; il faut ensuite multiplier ces deux probabilités ensemble, et par le produit des probabilités $\frac{1}{10}$ et $\frac{3}{10}$ que les témoins trompent; on aura ainsi $\frac{1}{350000}$ pour la probabilité de l'ćvénement observé, dans la seconde hypothese. Maintenant on aura la probabilité du fail attesté ou de la sortie du $n^{\circ} 1$, en divisant la probabilité relative à la première hypothèse, par la sommedes probabilités 
relatives aux denx hypothises; cette probabilité sera donc $\frac{2079}{1008}$; et la probabilité de la non sortie de ce numéro et du mensonge des témoins sera $\frac{1}{2080}$.

Si l'urne ne renfermait que les numéros 1 et 2 ; on trouverait de la même manière, $\frac{21}{22}$ pour la probabilité de la sortie du $n^{\circ} 1$, et par conséquent $\frac{T}{22}$ pour la probabilité du mensonge des témoins, probabilité quatre-vingtquatorze fois au moins, plus grande que la précédente. On voit par là, combien la probabilité du mensonge des témoins diminue quand le fait qu'ils attestent est moins probable en lui-même. En effet, on conçoit qu'alors laccord des témoins, lorsqu'ils trompent, devient plus difficile, à moins qu'ils ne s'entendent, ce que nous supposons ici ne pas avoir lieu.

Dans le cas précédent où l'urne ne renfermant que deux numéros, la probabilité $\dot{a}$ priori du fait attesté est $\frac{1}{2}$; la probabilité résultante des témoignages, est le produit des véracités des témoins, divisé par ce produit ajouté à celui des probabilités respectives de leur mensonge.

Il nous reste à considérer l'influence du temps, sur la probabilité des faits transmis par une chaine traditionnelle de témoins. Il est clair que cette probabilité doit diminuer à 
84

mesure que la chaîne se prolonge. Si le fait n'a aucune probabilité par lui-même; celle qu'il acquiert par les témoignages, décroît suivant le produit continu de la véracité des témoins. Si le fait a par lui-même, une probabilité; si, par exemple, ce fait est la sortie du $n^{\circ} 1$ d'une urne qui en renferme un nombre fini, et dont il est certain qu'on a extrait un seul numéro; ce que la chaìne traditionnelle ajoute à cette probabilité, décroît suivant un produit continu, dont le premier facteur est le rapport du nombre des numéros de l'urne moins un, à ce même nombre; ct dont chaque autre facteur est la vćracilé de chaque témoin, diminuce du rapport de la probabilité de son mensonge, au nombre des numéros de l'urne moins un; ensorte que la limite de la probabilité du fáit, est celle de ce fitit considéré $a$ priori ou indépendamment des témoignages, probabilité égale à l'unité divisée par le nombre des numéros de l'urne.

L'action du temps affaiblit donc sans cesse, la probabilité des faits historiques, comme elle altère les monumens les plus durables. On peut, à la vérité, la ralentir, en multipliant et conservant les témoignages et les monumens qui les étayent. L'imprimerie offre pour cet objet, un grand moyen malheureusement inconnu des anciens. Malgré les avantages 
infinis qu'elle présente; les révolutions physiques et morales dont la surface de ce globe sera toujours agitée, finiront, en se joignant à l'elfet inévitable du temps, par rendre douteux après des milliers d'années, les faits historiques aujourd'hui les plus certains.

Craig a essayé de soumettre au calcul, l'affaiblissement graduel des preuves de la religion chrćlienne : en supposant que le monde doit finir a l'époque où elle cessera d'čtre probable, il trouve que cela doitarriver, 1454 ans après le moment où il écrit. Mais son analyse est aussi fautive, que son hypolhèse sur la durée du monde est bizarre.

Les jugemens des tribunaux peuvent être assimilés aux témoignages, en considérant chaque juge, comme un témoin qui alteste la vérité de son opinion. Supposons le tribunal composé de trois juges. Si le jugement qu'ils prononcent, est rendu à l'unanimité, et si chacun d'eux mérite la même confiance; la probabilité de ce jugement sera la troisième puissance de la véridicité des juges, diviséc par cette puissance ajoutée à la troisième puissance de leur fuillibilité. Si le jugement n'est rendu qu'à la pluralité; sa probabilité sera cette véridicité elle-même, que l'on peut déterminer par l'expérience, en observant sur un très-grand nombre de jugemens, comlien 
ont été rendusà l'unanimité. $\mathrm{Si}$, par exemple, le rapport du second au premier de ces nombres, est celui de 7 à 16 ; on trouve par une analyse dont nous exposerons ci-aprés les principes, que la véridicité de chaque juge est $\frac{3}{4}$, et que la probabilité d'un nouveau jugement rendu à l'unanimité est $\frac{27}{28}$.

L'analyse confirme encore, ce que dicte le simple bon sens, savoir, que la bonté des jugemens est d'autant plus probable, que les juges sont plus nombreux et plus ćclairés; il importe done que les tribunaux d'appel remplissent ces deux conditions. Les tribunaux de première instance, plus rapprochés des justiciables, leur offirent l'avantage d'un premier jugement déjà probable, ct dont souvent ils se contentent, soit en transigeant, soit en se désistant de leurs prétentions. Mais si l'importance et l'incertitude de l'objet en litige, déterminent un plaideur à recourir an tribunal d'appel; il doit trouver dans une plus grande probabilité d'obtenir unjugement équituble, plus de sûreté pour sa fortune, et la compensation des cmbarras et des frais qu'une nouvelle procédure entraîne. C'est ce qui n'avait point lieu dans l'institution de l'appel réciproque des tribunaux de département, institution par là très-prćjudiciable aux intérêts des citoyens. 


\section{Des choix et des décisions des assemblées.}

La probabilité des décisions d'une assemblée dépend de la pluralité des voix, des lumières et de l'impartialité des membres qui la composent. Tant de passions et d'intecrêts particuliers y mêlent si souvent Ieur influence, qu'il est impossible de soumettre au calcul, cette probabilité. II y a cependant quelques résultats généraux dictés par le simple bon sens, etque le calcul confirme. Si, par exemple, İassemblée est très-peu éclairée sur l'objet soumis à sa décision; si cet objet exige des considérations délicates, ou si la vérité sur ce point est contraire à des préjugés reçus, ensorte qu'il y ait plus d'un contre un à parier que chaque votant s'en ćcartera; alors la décision de la majorité sera probablement mauvaise, et la crainte à cet ćgard sera d'autant plus juste, que l'assemblée sera plus nombreuse. Il importe donc à la chose publique, que les assemblées n'aient à prononcer que sur les objetsà la portée du plus grand nombre: il lui importe que l'instruction soit généralement répandue, et que de bons ouvrages fondés sur la ruison et l'expérience, éclairent ceux qui sont appelés à décider du sort de leurs semblables ou à les gouverner, et les 
prémunissent d'avance contre les faux aperçus et les préventions de l'ignorance. Les savans ont de fréquentes occasions de remarquer que les premiers aperçus trompent souvent, et que le vrai n'est pas tonjours vraisemblable.

Il est difficile de connaître et même de définir le voeu d'une assemblée, au milieu de la variété des opinions de ses membres. Essayons de donner sur cela, quelques règles, en considérant les deux cas les plus ordinaires, l'élection entre plusieurs candirlats, et celle entre plusieurs propositions relatives au même objet.

Lorsqu'une assemblée doit choisir entre plusieurs candidats qui se présentent pour une ou plusieurs places du même genre; ce qui parait le plus simple est de faire écrire à chaque votant sur un billet, les noms de tous les candidats, suivant l'ordre du mérite qu'il leur attribue. En supposant qu'il les classe de bonne foi, l'inspection de ces billets fera connaître les résultats des élections, de quelque manière que les candidats soient conparés entre eux; ensorte que de nouvelles élections ne peuvent apprendre rien de plus à cet égard. Il s'agit présentement d'en conclure l'ordre de préférence, que les billets établissent entre les candidats. Imaginons que l'on donne à chaque électeur, une urne qui contienne une 
infinilé de bonles au moyen desquelles il puisse nuancer tous les degrés de mérite des candidats: concevons encore qu'il tire de son urne, un nombre de boules proportionnel au mérite de chuque candidat, et supposons ce nombre écrit sur un billet, à còté du nom du candidat. Il est clair qu'en faisant une somme de tous les nombres relatifs à chaque candidat, sur chaque billet, celui de tous les candidats qui aura la plus grande somme, sera le candidat que l'assemblée préfère; et qu'en général, l'ordre de préférence des candidats, sera celui des sommes relatives à chacun d'eux. Mais les billets ne marquent point le nombre des boules que chaque ćlecteur donne aux candidats : ils indiquent seulement que le premier en a plus que le second, le second plus que le troisième, et ainsi de suite. En supposant donc au premier, sur un billet donné, un nombre quelconque de boules; toutes les combinaisons des nombres inférieurs, qui remplissent les conditions précédentes, sont également admissibles; et l'on aura le nombre de boules, relatif i chaque candidat, en faisant une somme de tous les nombres que chaque combinaison lui donne, et en la divisant par le nombre entier des combinaisons. Si ces nombres sont très-considérables, comme on doit le supposer paur 
qu'ils puissent exprimer toules les nuances du mérite; une analyse fort simple fait voir que les nombres qu'il faut écrire sur chaque billet à côté du dernier nom, de l'avant-dernier, etc., peuvent être représentés par la progression arilhmétique 1, 2, 5, etc. En écrivant donc ainsi sur chaque billet, les termes de cette progression, et ajoutant les termes relatifs à chaque candidat sur ces billets; les diverses sommes indiqueront par leur grandeur, l'ordre de préférence qui doit être établi entre les candidats. Tel est le mode d'élection, qu'indique la Théorie des Probabilités. Sans doute, il serait le meilleur; si chaque électeur inscrivait sur son billet, les noms des candidats, dans l'ordre du mérite qu'il leur attribue. Mais les intérêts particuliers et beaucoup de considérations étrangères au mérite, doivent troubler cet ordre, et faire placer quelquefois au dernier rang, le candidat le plus redoutable à celui que l'on préfere; ce qui donne trop d'avantage aux candidats d'unmédiocre mérite. A ussil'expérience a-t-elle fait abandonner ce mode d'élcction, dans les établissemens qui l'avaient adopté.

L'élection à la rnajorité absolue des suffrages réunit à la certitude de n'admettre aucun des candidats que cette majoritérejetterait, l'avantage d'exprimer le plus souvent, le voeu de 


\section{SUR TRS PRODABITITÉS.}

l'assemblée. Elle coïncide loujours avec le mode précédent, lorsqu'il n'y a que deux candidats. A la vérité, elle expose à linconvénient de rendre les élections interminables. Mais l'expérience a fait voir que cet inconvénient est nul, et que le desir général de mettre fin aux élections, rémit bientôt la majorité des suffrages sur un des candidats.

Le choix entre plusieurs propositions relatives au mème objet, semble devoir être assujéti aux mêmes règles, que l'élection entre plusieurs candidats. Mais il existe entre ces deux cas, cette difference, savoir, que le mérite d'un candidat n'exclut point celui de ses concurrens; au lieu que si les propositions entre lesquelles il faut choisir, sont contraires, la vérité de l'une exclut la vérité des autres. Voici comme on doit alors envisager la question.

Donnons à chaque votant, uno urne qui renferme un nombre infini de boules; et supposons qu'il les distribue sur les diverses prom positions, en raison des probabilités respectives qu'il leur attribue. Il est clair que le nombre total des boules, exprimant la certitude, et le votant étant par l'hypothèse, assuré que l'une des propositions doit être vraie; il répartira ce nombre en entier, sur les propositions. Le problème se réduit donc 
à déterminer les combinaisons dans lesquelles les boules seront réparties, de manière qu'il y en ait plus sur la premicre proposition du billet, que sur la seconde; pius sur la seconde que sur la troisième, etc.; à faire lẹs sommes de tous les nombres de boules, relatifs à chaque proposition dans ces diverses combinaisons; et à diviser cette somme, par le nombre des combinaisons : les quotiens seront les nombres de boules, que l'on doit attribuer aux propositions sur un billet quelconque. On trouve par l'analyse, qu'en partant de la derniére proposition, pour remonter à la première; ces quotiens sont entre eux, comme les quantités suivantes : $1^{\circ}$ l'unité $^{\circ}$ divisée par le nombre des propositions; $2^{\circ}$ la quantité précćdente augmentée de l'unité divisée par le nombre des propositions moins une; $3^{\circ}$ cette seconde quantité augmentée de l'unité divisée par le nombre des propositions moins deux; et ainsi du reste. On écrira done sur chaque billet, ces quantités à côté des propositions correspondantes; et en ajoutant les quantités relatives à chaque proposition, sur les divers billets; les sommes indiquerons par leur grandeur, l'ordre de préférence que l'assemblée donne à ces propositions. 
Des Lois de la Probabilité, qui résultent de la multiplication indéfinie des événemens.

Au milieu des causes variables et inconnues que nous comprenons sous le nom de hasard, et qui rendent incertaine et irrégulière, la marche des événemens; on voit naître à mesure qu'ils se multiplicnt, une régularité fiappante qui semble tenir à un dessein, et que l'on a considérée comme une preuve de la providence qui gouverne le monde. Mais en y réfléchissant, on recounaît bientôt que cette régularité n'est que le développement des possibilités respectives des érénemens simples, qui doivent se présenter plus souvent, lorsqu'ils sont plus probables. Concevons, par exemple, une urne qui renferme des boules blanches et des boules noires; et supposons qu'à chaque fois que l'on en tire une boule, on la remette dans l'urne pour procéder à un nouveau tiragc. Le rapport du nombre des boules blanches extraites, au nombre des boules noires extraites, sera le plus souvent très-irrégulier dans les premiers tirages; mais les causes variables de cette irrégularité, produisent des effets alternativement favorables et contraires à la marche 
régulière des événemens, et qui se détruisant mutuellement dans l'cnsemble d'un grand nombre de tirages, laissent de plus en plus apercevoir le rapport des boules blanches aux boules noires contenues dans l'urne, ou les possibilités respectives d'en extraire une boule blanche et une boule noire a chaque tirage. De là résulte le théorèrme suivant.

La probabilité que le rapport du noinbre des boules blanches extraites, au nombre total des boules sorties, ne s'écarte pas de la possibilité d'extraire une boule blanche à chaque tirage, au-delà d'un intervalle donné, approche indéfiniment de la ceritude, par la multiplicatiou indéinie des événemens, quelque petit que l'on suppose cet intervalle. Ce théorime indiqué par le bon sens, était difficile a démontrer par l'analysc. ${ }^{5}$ Aussi l'illustre géomètre Jacques Bernoulli qui s'en est occupé le premier, attachait-il une grande importance à la démonstration quî̀ en a donnée. Le calcul des fonctions génératrices, appliqué à cet objet, non-seulement démontre avec facilité ce théorème; mais de plus il donne la probabilité que le rapport des événemens observés, ne s'écarte que dans certaines limites, du vrai rapport de leurs possibilités respectives.

On peut tirer du ihéorème précédent, cette 
conséquence qui doit être regardée comme une loi générale, savoir, que les rapports des effets de la nature, sont à fort peu près constans, quand ces effets sont considérés en grand nombre. Ainsi, malgré ja varićcé des' années, la somme des productions pendant un nombre d'années, considérable, est sensiblement la même; ensorte que l'homme, par une utile prévoyance, peut se mettre à l'abri de l'irrégularité des saisons, en répandant également sur tous les temps, les bjens que la nature distribue d'une manière inégale. Je n'excepte pas de la loi précédente, les effets dus aux causes morales. Le rapport des naissances annuelles al la population, et celui des mariages aux naissances, n'éprouvent que cie très-petites variations: a Paris, le nombre des naissances annuelles a toujours étć lc mêne à peu près; et j'ai ouil dire qu'à la poste, dans les temps ordinajires, le nombre des lettres mises au rebut par les défauts des adresses, change peu chaque année.

Il suit encore de ce théoréme, que dans une série d'événemens, indéfiniment prolongée, l'action des causes régulières et constantes doit l'emporter à la longue, sur celle des causes irrégulières. C'est ce qui rend les gains des loteries, aussi certains que les produits de l'agriculture; les chances qu'elles se 
$9^{6}$

réservent, leur assurant un bénéfice daus l'ensemble d'un grand nombre de mises, $\Lambda$ insi des chances favorables et nombreuses étant constamment attachées à l'observation des principes éiernels de raison, de justice et d'humanité, qui fondent et maintienuent les sociétés; il y a un grand avantage à se conformer à ces principes, et de graves inconvéniens à s'en écarter. Que l'on consulte les histoires et sa propre expérience; on y verra tous les faits venir à l'appui de ce résultat du calcul. Considérez les avantages que la bonnefoi a procurés aux gouvernemens qui en ont fait la base de leur conduite, et comme ils ont été dédommagés des sucrifices qu'a pu leur coûter une scrupuleuse exactiiude à tenir leurs promesses : quel immense crédit au dedans! quelle prépondérance au dehors! Voyez au contraire, dans quel abîme de malheurs, les peuples ont été souvent précipités par l'ambilion et la perfidie de leurs chefs. Toutes les fois qu'une grande puissance enivrée de l'amour des conquêtes, aspire à la domination universelle; le sentiment de l'indépendance produit entre les nations injustement attaquées, une coalition dont etle devient presque toujours la victime. Parcillement, au milieu des causes variables qui étendent ou resserrent les divers états; les limites naturelles, 


\section{SUR LES PROBABILITES.}

en agissant comme causes constantes, doivent finir par prévaloir. Il importe donc à la stabilité comme au bonheur des empires, de ne pas les étendre au-delà de ces limites dans lesquelles ils sont ramenés sans cesse par l'action de ces causes; ainsi que les eaux des mers, soulevécs par de violentes tempêtes, retombent dans leurs bassins par la pesanteur. C'est encore un résultat du calcul des probabilités, contirmé par de nombreuses et funestes expériences. L'histoire traitée sous le - point de vue de l'influence des causes constantes, unirait à l'iutérêt de la curiosité, celui d'offrir aux hommes, les plus utiles leçons. Quelquefois on altribue les effets inévitables de ces causes, a des circonstances accidentelles qui.n'ont fait que développer leur action. 11 est, par exemplc, contre la nature des choses, qu'un peuple soit à jamais gouverné par un autre, qu'une vaste mer ou une grande dislance en sépare. On peut affirmer qu'à la longue, cette cause constante se joignant sans cesse aux causes variables qui agissent dans le même sens, et que la suite des temps développe, fuira par en trouver d'assez fortes pour rendre au peuple soumis, son indépendance nalurelle, ou pour le réunir à un état puissant qui lui soit couligu.

Dans un grand nombre de cas, et ce sont 
$9^{8}$

les plus importans de l'analyse des hasards, les possibilités des événemens simples sont inconnues, et nous sommes réduits à chercher dans les événemens passés, des indices qui puissent nous guider dans nos conjectures sur les causes dont ils dépendent. En appliquant l'analyse des fonclions génératrices, au principe exposé ci-devant, sur la probabilité des causes, tirée des événemens observés; on est conduit au théorème suivant.

Lorsqu'un événement simple ou composé de plusieurs événemens simples, tel qu'une parlie de jeu, a ceté répété un grand nombre de fois; les possibilités des événemens simples, qui rendeni ce que l'on a observé, le plus probable, sont celles que l'observation indique avec le plus de vraisemblance : à mesure que l'évémenient observése répète, cette vraisemblance augmente et finirait par se confondre avec la certitude, si le nombre des répétitions devenait infini.

Il y a ici deux sortes d'approximations; l'une d'elles est relative aux limiles prises de part et d'autre, des possibilités qui donnent au passé, le plus de vraisemblance : l'autre approximation se rapporte à la probabilité que ces possibilités tombent dans ces limites. La répétition de l'événement composé accroit de plus en plas cette probajilité, les limiles 


\section{SUR LES PROBABILITÉS.}

restant les mêmes : clle resserre de plus en plus l'intervalle de ces limites, la probabilité restant la même: dans l'infini, cet intervalle devient nul, et la probabilité se change en certitude.

Si l'on applique ce théoreme, au rapport des naissances des garçons à celles des filles, observé dans les diverses parlies de l'Europe; on trouve que ce rapport partout a peu prés égal à celui de 22 à 21 , indique avec une extrême probabilité, une plus grande facilité dans les naissances des garçons. En considérant ensuite qu'il est le même à Naples qu'à Pétersbourg, on verra qu'à cet égard, l'influcnce du climat est insensible. On piut donc soupconner conlre lopinion commune, que cette supériorité des naissances masculines subsiste dans l'orient même. J'avais en conséquence invité les savans français envoyés en Égypte, à s'occuper de cette question intéressante; mais la difficulté d'oblenir des renscignemens précis sur les naissances, ne leur a pas permis de la résoudre.

Le rapport des naissances des garçons à celles des tilles, différant très-peu de l'unité; des nombres même asse $z$ grands de naissances observées dans un lieu, pourraient offir à cet égard, un résultal conlraire à la loi générale, sans que l'on fút en droit d'en conclure 
que cette loi n'y existe pas. Pour tirer cette conséquence, il faut employer de très-grands nombres, et s'assurer qu'elle est indiquéc avec une grande probabilité. Buffon cite, par exemple, dans son Arithmétique politique, plusieurs communes de Bourgogne, où les naissances des filles ont surpassé celles des garcons. Parmi ces communes, celle de Carcelle-lc-Grignon préscnte sur 2009 naissances pendant cinq années, 1026 filles et 983 garcons. Quoique ces nombres soient considérables, cependant ils n'indiquent une plus grande possibilité dans les naissances des filles, qu'avec la probabilité $\frac{9}{10}$; et cette probabilité plus petite que celle de ne pas amener croix quatre fois de suite, au jeu de croix et pile, n'est pas suffisante pour rechercher la cause de cette anomalie qui, selon toute vraisemblance, disparaîtrait, si l'on suivait pendant un siecle, les naissances dans celie commune.

Les registres des naissances, que l'on tient avec soin pour assurer l'ćtat des ciloyens, peuvent servir à déterminer la population d'un grand empire, sans recourir au dénombrement de ses habitans, opération pénible et dilficile à faire avec exactitude. Mais il faut pour cela, connaitre le rapport de la population aux naissances annuelles. Le moyen d'y parvenir, le plus précis, consiste $1^{\circ}$ a 
choisir dans l'empire, des départemens distribués d'une manière à peu près égale sur toute sa surface, afin de rendre le résultat général, indépendant des circonstances locales; $2^{\circ}$ à dénombrer avec soin, pour une époque donnée, les habitans de plusieurs communes dans chacun de ces départemens; $3^{\circ}$ à déterminer par le relevé des naissances durant plusieurs années qui précèdent et suivent celte époque, le nombre moyen correspondant des naissances annuelles. Ce nombre divisé par celui des habitans, donnera le rapport des naissances annuelles à la population, d'une manière d'autant plus sùre, que le dénombrement sera plus considérable. Le gouvernement convaincu de l'utilité d'un semblable dénombrement, a bien voulu en ordonner l'exécution, à ma prièré. Dans trente départemens répandus également sur toute la France, on a fait choix des communes qui pouvaient fournir les renseignemens les plus précis. Leurs dénombremens ont donné 2057615 individus pour la somme totale de leurs habitans au 25 septembre 1802 . Le relevé des naissances dans ces communes pendant les années 1800 , 1801 et 1802 , a donné

\begin{tabular}{l|c|c} 
Naissances. & Mariages. & Décès. \\
0312 garçons. & 46037. & 105659 hommes. \\
09445 femmes.
\end{tabular}


Lc rapport de la population aux naissances arnuelles est donc $28 \frac{352845}{100000}$; il est plus grand qu'on ne l'avait estimé jusqu'ici. En multipiiant par ce rapport, le nombre des naissances annuclles en France, on aura la population de ce royaume. Mais quelle est la probabilité que la population ainsi déterminée, ne s'écartera pas de la véritable, au-dcla d'une limite donnée? En résolvant ce problème, et appliquantà sa solution, les données précédentes, j’ai trouvé que le nombre des naissances annuelles en France, étant supposé d'un million, ce qui porte sa population à 285,52845 habitans; il y a près de trois cent mille à parier contre un, que l'erreur de ce résultat n'est pas d'un demi-million.

Le rapport des naissances des garçons à celles des filles, qu'offre le relevé précédent, est celui de 22 a 21 ; et les mariages sont aux naissances, comme trois est à quatorze.

A Paris, les baptêmes des enfans des deux sexes s'écartent un peu du rapport de 22 à 21. Depuis 1745 , époque à laquelle on a commencé à distinguer les sexes sur les registres des naissances, jusqu'it la fin de 1784 , on a baptisé dans cette capitale, 395586 garçons et 377555 filles. Le rapport de ces deux nombres est à peu près celui de 25 à 24 ; il paraît donc qu'a Paris, une cause particulière rapproche 
de l'égalité, les baptêmes des deux sexes. Si l'on applique à cet objet, le calcul des probabilités; on trouve qu'il y a 238 à parier contre un, en faveur de l'existence de cette cause, ce qui suffit pour en autoriser la recherche. En y réfléchissant, il m'a paru que la diffèrence observée tient à ce que les parens de la campagne et des provinces, trouvant quelqu'avantage à retenir près d'eux les garçons, en avaient envoyé à l'hospice des EnfansTrouvés de Paris, moins relativement a:x filles, que suivant le rapport des naissances des deux sexes. C'est ce que le relevé des registres de cet hospice m’a prouvé. Depuis le commencement de 1745 jusqu'à la fin de 1809 , il y est entré 165499 garçons, et $15 \%$ / 05 filles. Le premier de ces nombres n'cxcede que d'un trente-huitième, le second qu'il aurait dû sưrpasser au moins d'un vingt-quatrième. Ce qui confirme l'existence de la cause assignée, c'est qu'en n'ayant point égard aux enfans trouvés, le rapport des naissances des garçons à celles des filles, est à Paris, comme dans le reste de la France, celui de 22 à 21 .

Laconstance de la supériorité desnaissances des garçons sur celles des filles, à Paris et à Londres, depuis qu'on les observe, a paru à quelques savans, être une preuve de la providence sans laquelle ils ont pensé que les causes irróguiières qui troublent sans cesse 
la marche des événemens, aurait dû plusieurs fois, rendre les naissances annuelles des filles, supérieures à celles des garçons.

Mais cette preuve est un nouvel exemple de l'abus gue l'on a fait si souvent des causes finales, qui disparaissent toujours par un examen approfondi des questions, lorsqu'on a les domnées nécessaires pour les résoudre. I a constance dont il s'agit, est un résultat des causes régulières qui donnent la supériorité aux naissances des garçons, et qui l'emportent sur les anomalies dues au hasard, lorsque le nombre des naissances annuelles est considérable. La recherche de la probabililé que cette conslance se maintiendra pendant un long espace de temps, appartient à celte branche de l'analyse des hasards qui remonte des événemens passés, à la probabilité des événemens futurs; et il en résulte qu'en partant des naissances observées depuis $17+5$ jusqu'en 1784 , il y a près de quatre i! purier contre un, qu'à Paris les naissances amue!'”s des girçons surpasseront conslamment peudant un siecle, les naissanees des filles; il $n^{\prime} y$ a done aucune raison de s'étonner que cela ait eu lieu pendant un demi-siècle.

bomons cncore un exemple du développement des rapports constans que les événemens présentent, à mesure qu'ils se multiplicnt. 
Concevons une série d'urnes disposées circulairement, et renfermant, chacune, un trèsgrand nombre de boules blanches et noires: les rapports des boules blanches aux noires, dans ces urnes, pouvant être très-différens à l'origine, et tels, par exemple, que l'une de ces urnes ne renferme que des boules blanches, tandis qu'une autre ne contient que des boules noires. Si l'on tire une boule de la première urne, pour la mettre dans la seconde; qu'après avoir agité cette seconde urne, afin de bien mêler la boule ajoutée, avec les autres, on en tire une boule pour la mettre dans la troisieme urne, et ainsi de suite jusqu'a la dernière urne dont on extrait une boule, pour la mettre dans la première, et que l'on recommence indéfiniment cette série de tirages; l'analyse des probabilités nous montre que les rapports des boules bianches aux noires, dans ces urnes, finiront par être les mêmes. et égaux au rapport de la somme de toutes les boules blanches, à la somme de toutes les boules noires contenues dans les urnes. Ainsi par ce mode régulier de changement, Pirrégularité primitive de ces rapports, disparaît à la longue, pour faire place à l'ordre le plus simple. Maintenant si entre ces urnes, on en intercale de nouvelles dans lesquelles le rapport de la somme des boules blanches, 
à la somme des boules noires qui elles contiennent, diffëre du précédent; en continuant indéfiniment, sur l'ensemble de ces urnes, les extractions que nous venons d'indiquer; l'ordre simple établi dans les ancicnnes urnes sera d'abord troublé, et les rapports des boules blanches aux boules noires deviendront irréguliers ; mais peu à peu, cette irrégularité disparaîtra pour faire place à un nouvel ordre, qui sera cnfin celui de l'égalité dos rapports des boules blanches aux boules noires contenues dans les urnes. On peut étendre ces résultats, à toutes les combinaisons de la nature, dans lesquelles les forces constantes qui animent les êtres dont clles sont formées, établissent des modes réguliers d'action et de changement.

Les phénomènes qui semblent le plus dépendre du hasard, présentent donc en se multipliant, une tendance à se rapprocher sans cesse, de rapports fixes; de manière que si l'on conçoit de part et d'autre de chacun de ces rapports, un intervalle aussi petit que l'on voudra, la probabilité que le résultat moyen des observations tombe dans cet intervalle, finira par ne différer de la certitude, que d'une quantité au-dessous de toute grandeur assignable. On peut ainsi par le calcul des probabilités, appliqué à un grand nombre 


\section{SUR LES PROBABILITĹLS.}

d'observations, reconnaitre l'existence de ces rapports. Mais avant que d'en rechercher les causes, il est nécessaire, pour ne point s'égarer dans de vaines spéculations, de s'assurer qu'ils sont indiqués avec une probabilité qui ne permet point de les regarder comme des anomalies dues au hasard. La théorie des fonctions génératrices donne une expression très-simple de cette probabilité, que l'on obtient en int'́grant le produit de la différentielle de la quantité dont le résultat déduit d'un grand nombre d'observations s'écarte de la vérité, par une constante moindre que l'unité, dépendante de la nature du problème, et élevée à une puissance dont l'exposant est le rapport du carré de cet écart, au nombre des observations. Lintégrale prise entre des limites données, et divisće par la même intégrale étendue à l'infini positif et négatif, exprimera la probabilité que l'écart de la vérité, est compris entre ces limites. Telle est la loi générale de la probabilité des résultats indiqués pur un grand nombre d'observations.

Du Calcul des Probabilités, appliqué à la recherche des phénoménes et.de leurs causes.

Les phénomènes de la nature sont le plus souvent enveloppés de tant de circonstances 
étrangères, un si grand nombre de causes perturbatrices y mêlent leur influence; qu'il est très-difficile, lorsqu'ils sont fort petits, de les reconnaitre. On ne peut alors y parvenir, qu'en multipliant les observations; afin que les effets étrangers venant à se détruire, les résultats moyens mettent en évidence ces phénomènes. On conçoit par ce qui précède, que cela n'a lieu rigoureusement que dans le cas d'un nombre infini d'observations: dans tout autre cas, les phénomènes ne sont indiqués par les résultats moyens, qu'avec une probabilité d'autant plus forte, que Ies observalious sont en plus grand nombre, et dont il importe d'apprécier la valeur.

Prenons pour exemple, la variation diurne de la pression de l'atmosphère à l'équateur oir elle est le plus sensible, et le plus facile à reconnaître, les changemens irréguliers du baromètre y étant plus considérables. On remarqua bientôt dans les hauteurs qu'ilindique, une petite oscillation diurne dontle maximum a lieu vers neuf heures du matin, et le $m i-$ nimum vers quatre heures du soir : un sccond maximum a lieu vers onze heures du soir, et le second minimum vers quatre heures du matin: les oscillations de la nuit sont moiridres que celles du jour, dont l'étendue est de deux millimètres. L'inconstance 
de nos climats n’a point dérobé celte variation à nos observateurs, quoiqu'elle y soit moins sensible qu'entre les tropiques. En appliquanl l'analyse des probabilités, aux observations nombreuses et précises faites par Ramiond, pendant plusieurs années consécutives; je trouve qu'clles indiquent l'existence el la quantité de ce phénomène, de manicire à ne laisser aucun doute. La période de sa variation élant d'un jour solaire; sa cause est évidomment la chaleur que le soleil communicue aux diverses parties de l'atmos phère; quoiqu'il soit presqu'impossible d'en cálculer les effets. Cel as tre agit encore par son altraction, sur ce fluide: il y produit avec la lune, des oscillations semblables à celles du flux et du reflux de la mer, oscillations dont jai délerminé les lois daus la Mécanique céleste, et qui scront, un jour, reconnues par des observations nombreuses faites à l'équateur avec d'excellens barometres.

On peut encore rar l'analyse des probabilités, vérifier l'existence ou l'influence de certaines causes dont on a cru remarquer l'action sur les êtres organisés. De tous les instrumens que nous pouvons employer pour connaître les agens imperceptibles de la nature, les plus sensibles sont les nerfs, surtout lorsque des causes palticulières exaltent leur sensibilité. 
C'est par leur moyen, qu'on a découvert la faible électricité que développe le contact de deux métaux hétérogènes; ce qui a ouvert un champ vaste aux recherches des physiciens et des chimistes. Les phénomènes singuliers qui résultent de l'extrême sensibilité des nerfs dans quelques individus, ont donné naissance à diverses opinions sur l'existence d'un nouvel agent que l'on a nommé magnétisme animal, sur l'action du magnétisme ordinaire et l'influence du soleil et de la lune, dans quelques affections nerveuses; enfin sur les impressionsque peut fuire naître la proximité des métaux ou d'une eau courunte. II est naturel de penser que l'action de ces causes est très-faible, et qu'elle peut être facilement troublée par un grand nombre de circonstances accidentelles. Ainsi, parce qu'clle ne s'est point manifestée dans quelques cas, on ne doit pas rejeter son existence. Nous sommes si éloignés de connaître tous les agens de la nature, et lcurs divers modes d'action; qu'il ne serait pas philosophique de nier les phénomènes, uniquement parce qu'ils sont inexplicables dans l'état actuel de nos connaissances. Seulement, nous devons les examiner avec une attention d'autant plus scrupuleuse, qu'il parait plus difficile de les admeltre; et c'est ici que le calcul des probabilités äcrient 
indispensable, pour déterminer jusqu’à quel point il faut multiplier les observations on les expériences, afin d'obtenir en faveur des agens qu'elles indiquent, une probabilité supérieure aux raisons que l'on peut avoir d'ailleurs, de ne pas les admettre.

Le calcul des probabilités peut faire apprécier les avantages et les inconvéniens des méthodes employées dins les sciences conjecturales. Ainsi, pour reconnaître le meilleur des traitemens en usage dans la guérison d'une maladie, il suffit d'éprouver chacund'eux sur un même nombre de malades, en rendant toutes les circonstances parfaitement semblables. La supériorilé du traitement le plus avantageux se manifestera de plus en plus, à mesure que ce nombre s'accroitra; et le calcul fera connaître la probabilité correspondante de son avantage. Le même calcul s'étend encore aux objets de l'économie politique, pour laquelle les opérations des gouvernemens sont autantd'expćriences en grand, propres à les éclairer sur la conduite quils doivent tenir dans les cas semblables à ceux qui se sont déjà présentés. Tant de causes imprévues ou cachées ou inappréciables influent sur les institutions hum:ines, qu'il est impossible d'en juger à priori, les résultats. Une longue suite d'expériences développe les 
effets de ces causes, et indique les moyens de remédier à ceux qui sont nuisibles. On a souvent fait à cet égard, des lois sages; mais parce que l'on avait négligé d'en conserver les motifs, plusieurs ont été abrogées comme inutiles, et il a fallu pour les rétablir, que de fầcheuses expériences en airnl fait de nouveau, sentir le besoin. Il est donc bien important de tenir dans chaque branche de l'administration publique, un registre exactdes résultats qu'ont produits les divers moyens dont on a fait usage. Appliquons aux sciences politiques et morales, la méthode fondée sur l'observation et le calcul, méthode qui nous a si heureusement servi dans les sciences naturelles. Ne changeons qu'arec une circonspection extrême, nos anciennes institutions et les usages auxquels nos opinions et nos habitudes se sont depuis long-temps pliées. Nous connaissons bien par l'expérience du passé, les inconvéniens qu’ils présentent; mais nous ignorons quelle est l'étendue des maux que leur changement peut produire.

La considération des probabilités, étendue à l'astronomie, peut servir à reconnaitre la cause des anomalies observées duns les mouvemens cólestes, et à démêler les petites inégalités enveloppées dans les errcurs dont les observations sont susceptibles. Ce fut on 


\section{SUR LES PROBABILITÉS.}

comparant entre elles toutes ses observalions; que Ticho-Brahé reconnut la nécessité d'appliquer à la lune, une équation du temps, differente de celle que l'on appliquait au soleil ct aux planètes. Ce fut encore dans le résultat d'observations nombreuses, que Mayer aperçut pour la lune, une diminution dans le coefficieut de l'inćgalité de la précession, relatif aux autres corps célestes. Mais conme cette diminution ne semblait pas résulter de la gravitation universelle; la plupart des astronomes la négligèrent dans leurs calculs. Ayant soumis à l'analyse des probabilités, un grand nombre d'observations lumaires choisies dans cette vue, et que Bouvard voulut bien calculer à ma prière; elle me parut indiquée avec une si forte probabilicé, que je crus devoir en rechercher la causc. Je vis bientôt qu'elle ne pouvait être que l'ellipticité du sphéroïde terrestre, négligée jusqu'alors dans la théorie du mouvement lunaire, comme ne devant y produire que des termes insensibles : j'en conclus que ces termes deviennent sensibles par les intégrations successives des équations différentielles. Je déterminai donc ces termes par une analyse particulière, et je découvris d'abord l'inégalité du mouvement lunaire en latitude, qui est proportionnelle au sinus de la longitude de la lune, et qu'aucun astronome 
n'avait encore aperçue. Je recomus ensuitc au moyen de cetle inégalité, que la théoric de la pesanteur donne en effet la diminution indiquée par Mayer, dans l'équation de la précession, applicable à la lune. La quantité de celte diminution, et le coefficient de l'inégalité précédente en latitude, sont très-propres a fixer l'aplatissement de la terre. Ayant fait part de mes recherches, à Burg qui s'occupait alors à perfectionner les tables de la lune, par la comparaison de toutes les bonnes observations; je le priai de determiner avec un soin particulicr, ces deux quantités. Par un accord tres-remarquable, les valeurs qu'il a trouvécs, donment à la terre, le même aplatisscment $\frac{1}{305}$, aplatissement qui diflère peu du milicu conclu des mesures des degrés du méridien el du pendule; mais qui, vu l'influence des erreurs des observations et des causes perturbatrices, sur ces mesures, me paraît plus exactement déterminé par ces inćgalités lunaires.

Le calcul des probabilités m’a conduit pareillement à la cause des grandes irrégularités de Jupiter et de Suturne. En comparant les observations modernes aux anciennes, Halley trouva une accélération dans le mouvement de Jupiter, et un ralentissement dams celui de Saturne. Pour concilier les observations, 
il assujélit ces mouvemens, à deux équuations séculaires de signes contraires, et croissantes comme les carrés des temps écoulés depuis 1700. Euler et Lagrange soumirent à l'analyse, les altérations que devoit produire dans ces mouvemens, l'attraction mutuelle des deux planètes. Ils y trouvèrent des équations séculaires; mais leurs résultats étaient si diff'́rens, que l'un d'cux, au moins, devait être erroné. Je me déterminai donc à reprendre ce problème important de la mécanique céleste, et je reconnus l'invariabilité des moyens mouvemens planétaires; ce qui fit disparaître les équations séculairesintroduites par Halley, dans les tables de Jupiter et de Saturne. Il ne restait ainsi, pour expliquer les grandes irrégularités de ces planétes, que les attractions des comètes auxquelles plusieurs astronomes eurent effectivement recours, ou l'existence d'une inégalité à longue période, produite dans les mouvemens des deux planètes par leur action réciproque, et affectée de signes contraires, pour chacune d'elles. Un théorème que je trouvai sur les inégalités de ce genre, me rendit cette inégalitẹ́, très-vruisemblable. Suivant ce théorème, si le mouvement de Jupiter s'accélère, celui de Saturne se ralentit, ce qui est déjà conforme à ce que Halley avait remarqué; mais de plus, l'accélération 
de Jupiter, résultante du même théorìme, est au ralentissement de Saturne, à trés-peu prés dans le rapport des équations séculaires proposées par Halley. En considérant les moyens mouvemens de Jupiter et de Saturne, il me fut aisé de reconnaitre que deux fois celui de Jupiter, ne surpasse que d'une trèspetite quantité, cinq fois celui de Saturne. La période d'une inégalité qui aurait cet argument, serait d'environ neuf sićcles. A la vérité, son coefficient serait de l'ordre des cubes des excentricités des orbites; mais je savais qu'en vertu des intégrations successives, il acquicrt pour diviseur, le carré du très-petit multiplicateur du temps dans l'argument de cette inégalité, ce qui peut lui donner une grande valeur; il me parut done très-probable que celte inégalité a lieu. La remarque suivante accrut encore sa probabilitć. En supposant son argument nul, vers l'époque des observations de Ticho-Brahé; je vis que Halley avait dù trouver par la comparaison des observations modernes aux anciennes, les altérations qu'il avait indiquées; tandis que la comparaison des observations modernes entre elles, devait offrir des altérations contraires, et pareilles à celles que Lambert avait conclues de cette comparaison. L'existence de cette inégalité me parut donc 
extrêmement vraisemblable, et je n'hésitai point à entreprendre-le calcul long et pénible, nécessaire pour m'en assurer. Elle fut entièrement confirmée par le résultat de ce calcul qui, de plus, me fit connaître ungrand nombre d'autres inégalités dont l'ensemble a porté les tables de Jupiter et de Saturne, à la précision des observations mêmes.

Ce fut encore au moyen du calcul des probabilités, que je reconnus la loi remarquable des mouvemens moyens des trois premiers satellites de Jupiter, suivant laquelle la longitude moyenne du premier, moins trois fois celle du second, plus deux fois celle du troisième est rigoureusement égale à la demicirconférence. L'approximation avec laquelle les moyens mouvemens de ces astres satisfoni: à cette loi depuis leur découverte, indiquait son existence avec une vraisemblance extrême; j'en cherchai donc la cause, dans l'action mutuelle de ces trois corps. L'examen approfondi de cette action, me fit yoir qu'il a suffi qu'a l'origine, les rapports de leurs moyens mouvemens aient approché de cette loi, dans certaines limites, pour que leur action mutuelle l'ait établie et la maintienne en rigueur.

On voit par là, combien il faut être attentif aux indications de la nature, lorsqu'clles sont 
le résultat d'un grand nombre d'observations; quoique d'ailleurs; elles soient inexplicables par les moyens connus. L'extrème difficulté des problèmes relatifs au système du monde, a forcé les géomètres de recourir à des approximations qui laissent toujours a craindre que les quantités négligées r'aient une influence sensible. Lorsqu'ils ont été avertis de cette influence, par les observations; ils sont revenus sur leur analyse: en la rectifiant, ils ont toujours retrouvé la cause des anomalies observées; ils en ont déterminé les lois, et souvent, ils ont devancé l'observation, en découvrant des inégalités qu'elle n'avait pas encore indiquées. Ainsi l'on peut dire que la nature elle-même a concouru à la perfection des théories fondées sur le principe de la pesanteur universelle; et c'est, à mon sens, une des plus fortes preuves de la vérité de ce principe admirable.

L'un des phénomènes lesplus remarquables du système du monde, est celui de tous les mouvemens de rotation et de révolution des planctes et des satellites, dans le sens de la rotation du soleil, et à peu près dans le plan de son équateur. Un phénomène aussi remarquable n'est point l'effet du hasard : il indique une cause générale qui a déterminé tous ces mouvemens. Pour avoir la probabilité avec 


\section{SUR I.ES PROB̈ABILITÉS.}

laquelle cette cause est indiquée; nous observerons que le système planétaire tel que nous le connaissons aujourd'hui, est composé d'onze planetes et de dix-huit sateliites. On a reconnu les mouvemens de rotation du soleil, de six planetes, des satellites de Jupiter, de l'anneau de Saturne, et d'un de ses satellites. Ces mouvemens forment avec ceux de révolution, un ensemble de quarante-trois mouvemens dirigés dans le même sens; or on trouve par l'analyse des probabilités, qu'il y a plus de quatre mille milliards à parier contre un, que cette disposition n'est pas l'effet du hasard; ce qui forme une probabilité bien supérieure à celle des événemens historiques sur lesquels on ne se permet aucun doute. Nous devons donc croirc, au moins avec la mème confiance, qu'une cause primitive a dirigé les mouvemens planélaires; surtout si nous considérons que l'inclinaison du plus grand nombre de ces mouvemens à l'équateur solaire, est fort pelite.

Un autre phénomène également remarquable du systéme solaire, est le peu d'excentricité des orbes des planètes et des satellites, tandis que ceux des comèles sont très-alongés: les orbes de ce systène n'offrant point de suances intermédiaires entre une grande et. une petite excentricité. Nous sommes encore 
forcés de reconnaître ici l'effet d'une cause régulière : le hasard n'eût point donné une forme presque circulaire aux orbes de toutes les planètes et de leurs satellites; il est donc nécessaire que la cause qui a déterminé les mouvemens de ces corps, les ait rendus presque circulaires. Il faut encore que les grandes excentricités des orbes des comètes résultent de l'existence de cette cause, sans qu'elle ait influé sur les directions de leurs mouvemens; car on trouve qu'il y a presque autant de comètes rétrogrades, que de comètes directes, et que l'inclinaison moyenne de tous leurs orbes, approche très-près d'un demi-angle droit, comme cela doit être, si ces corps ont été lancés au hasard.

Quelle que soit la nature de la cause dont il s'agit; puisqu'elle a produit ou dirigé les mouvemens des planètes, il faut qu'elle ait embrassé tous ces corps; et vu les distances qui les séparent, elle ne peut avoir été qu'un fluide d'une immense étendue: pour leur avoir donné dans le même sens, un mouvement presque circulaire autour du soleil, il faut que ce fluide ait environné cet astre, comme une atmosphère. La considération des mouvemens planétaires nous conduit donc à penser qu'en vertu d'une chaleur excessive, l'atmosphère du soleil s'est primitivement 
étendue au-delà des orbes de toutes les planètes, et qu'elle s'est retirée successivement jusqu'à ses limites actuelles.

Dans l'état primitif où nous supposons le soleil, il ressemblait anx nébuleuses que le télescope nous montre composées d'un noyau plus ou moins brillant, entouré d'une nébulosité qui, en sc condensant à la surface du noyau, doit le transformer, un jour, en étoile. Si l'on conçoit par analogie, toutes les étoiles formées de cette maniere; on peut imaginer leur état antérieur de nébulosité, précédé luimême par d'autres états dans lesquels la inatière nébuleuse était de plus en plus diffuse, le noyau étant de moins en moins lumineux ct dense. On arrive ainsi, en remontant aussi loin qu'il est possible, à une nébulosité tellement diffuse, que l'on pourrait à peine en soupçonner l'existence.

Tel est, en effet, le premier état des nébuleuses que Herschel a observées avec un soin particulier, au moyen de ses puissans télescopes, et dans lesquelles il a suivi les progres de la condensation, non sur une seule, ces progrès ne pouvant devenir sensibles pour nous, qu'aprés des siècles, mais sur leur ensemble; a peu près comme on peut dans une vaste forêt, suivre l'accroissement des arbres sur les individus de divers âges, qu'elle. 
renferme. Il a d’abord observé la matičre nébuleuse répandue en amas divers, dans les différentes parties du ciel dont elle occupe une grande étendue. Il a vu dans quelquesuns de ces amas, cette matière faiblement condensée autour d'un ou de plusieurs noyaux peu brillans. Dans d'aulres nébuleuses, ces noyaux brillent davantage, relativement à la nébulosité qui les environne. Les atmospheres de chaque noyau, venant a se séparer par une condensation ultérieure, il en résulte des nébuleuses multiples formées de noyaux brillans très-voisins, et environnés, chacun, d'une atmosphère : quelquefois, la matière nébuleuse en se condensant d'une manière uniforme, a produit les nébuleuses que l'on nomme planétaires. Enlin, un plus grand degré de condensation transforme toutes ces nébuleuses, en étoiles. Les nébuleuses classées d'après cette vue philosophique, indiquent avec une extrême vraisemblance, leur transformation future en étoiles, ct l'état antérieur de nébulosité, des étoiles existantes. Les considérations suivantes viennent à l'appui des preuves tirées de ces analogies.

Depuis long-temps, la disposition particulière de quelques étoiles visibles à la vue simple, a frappé des observateurs philosophes. Mitchel a déjà remarqué combien il est peu 
probable que les étoiles des Pléiades, par exemple, aient été resserrées dans l'espace ćtroit qui les renferme, par les seules chances du hasard; et il en a conclu que ce groupe d'étoïles, et les groupes semblables que le ciel nous présente, sont les effets d'une cause primitive, ou d'une loi générale de la nature. Ces groupes sont un résultat nécessairc de la condensation des nébulcuses à plusieurs noyaux; car il est visible que la matière nébulcuse étant sans cesse attirée par ces noyaux divers; ils doivent former i la longue un groupe d'étoiles, pareil à celui des Plëiades. La condensation des nébuleuses à deux noyaux forme semblablement des étoiles très-rapprochées tournant l'une autour de l'autre, pareilles à celles dont Herschel a déjà considéré les mouvemens respectifs. Telles sont encore la soixante-unième du Cygne et sa suivante, dans lesquelles Bessel vient de reconnaître des mouvemens propres, si considérables et si peu différens, que la proximité de ces astres culre eux, et leur mouvement autour de leur centre commun de gravité, ne doivent laisscr aucun doute. Ainsi, l'on descend par les progrès de condensation de la matière nébuleuse, a la considération du soleil environné autrcfois d'une vaste atmosphère, considération à laquelle on remonte, comme on l'a vu, par 
l'examen des phénomènes du système solaire. Une rencontre aussi remarquable donne à l'existence de cet état antérieur du soleil, une probabilité fort approchante de la certitude.

Mais comment l'atmosphère solaire a-telle déterminé les mouvemens de rotation et de révolution des planètes et des sateliites? Si ces corps avaient pénétré profondément dans cette atmosphère, sa résistance les aurait fait tomber sur le soleil; on est donc conduità croire avec beaucoup de vraisemblance, que les planètes ont été formées aux limites successives de l'atmosphère solaire qui en se resserrant par le refroidissement, a dù abandonner dans le plan de son équateur, des zônes de vapeurs, que l'attraction mutuelle de leurs molécules a changées en divers sphéroïdes.

J'ai développé avec étendue, dans mon Exposition du Système du Monde, cette hypothèse qui me paraît satisfaire à tous les phénomènes que ce système nous présente.

Dans cette hypothèse, les comètes sont étrangères au système planétaire. En attachant leur formation, à celle des nébuleuses; on peut les regarder comme de petites nébuleuses à noyaux, errantes de systèmes en systèmes solaires, et formées par la condensation de la matière nébuleuse répandue avec. 
tant de profusion dans l'univers. Lcs comètes seraient ainsi par rapport à notre système, ce que les aćrolithes sont relativement à la terre, ia laquelle ils paraissent étrangers. Lorsque ces astres deviennent visibles pour nous, ils offrent une ressemblance si parfiite avec les nébuleuses, qu'on les confond souvent avec elles; et ce u'est que par leur mouvement, ou par la connaissance de toutes les nébuleuses renfermées dans la partie du ciel où ils se montrent, qu'on parvient à les en distinguer. Cette supposition explique d'une manière heureuse, la grande extension que prennent les têtes et les queues des comètes, à mesure qu'elles approchent du soleil, et l'extrême rareté de ces queues qui malgré leur immense profondeur, n'affuiblissent point sensiblement l'éclat des étoiles que l'on voit à travers.

Lorsque de petites nébuleuses parviennent dans la partie de l'espace oú l'attraction du soleil est prédominante, et que nous nommerons sphère d'activilé de cet astre; il les force à décrire des orbes elliptiques ou hyperboliques. Mais leur vitesse étant également possible suivant toutes les directions, elles doivent se mouvoir indifféremment dans tous les sens et sous toutes les inclinaisons à l'écliptique; ce qui est conlorme à ce que l'on observe. La grande excentricitédes orbes cométaires, 
résulte encore de l'hypothèse précédente. En effet, si ces orbes sont elliptiques, ils sont très-alongés; puisque leurs grands axes sont au moins égaux au rayon de la sphere d'activité du soleil. Mais ces orbes peuvent être hyperboliques, et si les axes de ces hyperboles ne sont pas trés-grands par rapport á la moyenne distance du soleil à la terre, le mouvement des comites qui les décrivent, paraîtra sensiblement hyperbolique. Cependant sur cent comètes dont on a déja les élémens, aucune n'a paru se mouvoir dans une hyperbole; il faut donc que les chances qui donnent une hyperbole sensible, soient extrêmement rares par rapport aux chances contraires.

Les comètes sont si petites, que pour devenir visibles, leur distance périhćlie doit être peu considérable. Jusqu’a présent cetle distance n'a surpassé que deux fois, le diamètre de l'orbe terrestre, et le plus souvent, elle a été au-dessous du rayon de cet orbe. On conçoit que pour approcher si près du soleil, leur vitesse au momenc de leur entrée dans sa sphère d'activité, doit avoir une grandeur et une direction, comprises dans d'étroites limiles. En déterminant par l'analyse des probabilités, le rapport des chances qui dans ces limites, dounent une hyperbole sensible, aux 
chances qui donnent un orbe que l'on puisse confondre avec une parabole; j'ai trouvé qu'il y a six mille au moins, à parier contre l'unité, qu'une nébuleuse qui pénètre dans la sphère d'activilé du soleil, de manière à pouvoir être observée, décrira ou une ellipse très-alongée, ou une hyperbole qui par la grandeur de son axe, se confondra sensiblement avec une parabole, dans la partie que l'on observe; il n'est doinc pas surprenant que jusqu'ici, l'on n'ait point reconnu de mouvemens hyperboliques.

L'attraction des planètes, et peut-être cncore la résistance des milieux éthérés, a dû changer plusieurs orbes comćtaires, dans des cllipses dont le grand axe est moindre que le rayon de la sphère d'activité du soleil; ce qui augmente les chances des orbes elliptiques. On peut croire que ce changement a eu lieu pour la comète de $168_{2}$, la seule dont on ait jusqu’a présent, déterminé la révolution.

Des milieux qu'il faut choisir entre les résultals d'un grand nombre d'observations.

La recherche de ces milieux est très-importante dans la philosophie naturelle; et l'analyse qu'elle exige, est la plus délicate et la plus épineuse de toute la théorie des 
probabilités. Les obscrvations et les expériences les plus précises sont toujours sujettes à des erreurs qui influent sur la valeur des élémens que l'on veut en déduire. Pour faire disparaitre ces erreurs, autant qu'il est possible, en les détruisant les unes par les autres; on multiplie les observations dont le résultat moyen est d'autant plus exact, que leur nombre est plus considérable. Mais quelle est la manière la plus avantageuse de former ce résullat moyen? De quelle erreur ce résultat est-il encore susceptible? C'est ce que l'analyse des probabilités peut seule faire connaitre; et voici ce qu'elle nous apprend.

Pour fixer les idées, supposons que l'on cherche à déterminer par l'observation, la grandeur apparente d'un disque vu d'une dis tance donnée. Si l'on a pris un grand nombre de mesures du disque avec des instrumens semblables, et à une même distance de ce disque; on aura sa grandeur moycnne apparente, en divisant la somme de toutes les mesures partielles, par le nombre de ces mesures. Pour avoir l'erreur moyenne à craindre en plus ou en moins, sur ce résultat; nous observerons que cette erreur est la somme des produits de chaque erreur possible, par sa probabilité. Une crrcur, soil positive, soit négative, devant être considérée comme une 
perte aujeu, on doitévaluerl'crreur moyenne, comme on évalucrait une perte moyenne. En déterminant par l'analyse des fonctions génératrices, l'expression de cette errcur; on trouve qu'elle a pour facteur, une quantité dépendante de la loi de probabilité des erreurs de chaquemesure. Cette loi nous est inconnue: seulement, il est naturel d'admettre que les erreurs négatives sont aussi probables que les positives; il semble donc impossible d'évaluer cette erreur moyenne. Mais en déterminant par la même analyse, la somme des carrés des erreurs des observations; j'ai reconnu qu'elle a le même facteur. De là, j’ai conclu la règle suivante.

Si l'on prend les différences entre le résultat moyen de toutes les mesures, et chacune, d'elles; l'errear moyenne à craindre en plus ou en moins sur ce résultat, est une fraction dont le numćrateur est la racine carrée de la somme des carrés de ces différences, et dont le dínominateur est le produit du nombre des mesures, par la racine carrée du rapport de la circonférence au rayon.

On a ainsi le résultat moyen le plus avantageux, et l'on peut en apprécier l'exactilude. Pour rapporter ensuite ce résultat, à la dislance donnće; il suffit de le mulliplier par 
le rapport inverse de cette distance, à celle d'oà les mesures ont élé prises.

Supposons maintenant que l'on ait pris ces mesures, à différentes distances; et que l'on veuille toujours en conclure la grandeur apparente du disque vu d'une distance donnée. Il est clair que l'crreur de chaque observation aura d'autant moins d'influence, que l'observation aura été faite plus près du disque; il est encore facile de voir que chaque mesure observée, moins son erreur, doit être égale à la grandeur que l'on cherche, mullipliée par le rapport de la distance donnée, à la distance d'ou la mesure a été prise. En considérant la grandeur cherchée, comme une inconnue; chaque mesure observéc donnera une équation du premicr degré dont le premier membre scra le produit de l'inconnue, par ce rapport; et dont le second membre sera la mesure observée, moins son crreur. Si l'on ajoute toutes ces équations, leur ensemble formera une équation finale qui, en supposant nulle, la somme des erreurs de toutes les observations, domera une valeur de l'inconnue, à laquelle toutes les observations auront concouru, et qui par là, doit avoir une grande précision. C'est la règle que l'on suit communément; mais elle ne donne pas le résultat le plus 
avantageux, celui qui ne laisse à craindre que la plus pelite errear moyenne. Pour avoir ce résultat, on doit observer que toutes les maniéres possibles de combiner les équations précédentes, afin d'obtenir une équation finale du premier degré, qui détermine l'inconnue, reviennent à les multiplier, chacune, par un facteur, ct à les ajouter ensuite sans avoir égard aux erreurs des observations. En prenant donc pour ces facteurs, des constantes arbitraires, et cherchant l'expression analytique de l'erreur moyenne du résultal donné par l'équation finale; ii faut déterminer les conslantes, ensorle que cette erreur soit un minimum. On trouve alors que chaque constante est égale au coefícient de l'inconnue, dans l'équation partielle qu'elle multiplie; la valeur de linconnue, donnée par l'équation finale, est ainsi exprimée par une fraction qui a pour numérateur, la somme des produits du coefficient de linconnue dans chaque équation partielle, par la mesure observéc correspondante; et pour dénominateur, la somme des carrés de tous ces coefficiens. Si l'on prend ensuite les différences entre les mesures observées, et les produits successifs de ce résultat par les coefficiens de l'inconnue dans les équations partielles; l'erreur moyenne qu'il laisse encore à craindre, sera 
la racine carrée d'une fraction dont le numés rateur est la somme des carrés de ces differences, et dont le dénominateur est le produit de ces trois quancités, savoir, le nombre des observations, la somme des carrés des cocfficiens de l'inconnue, dans les équations partielles, et la circonférence dont le rayon est l'unité.

Il est facile de voir que si l'on élève au carré, l'expression de l'erreur de chaque thesure, tirće de l'équation partielle correspondante; si l'on rend ensuite, un minimum, la somme de ces carrés, en y faisant varicr l'inconnue; l'équation du minimum donnera pour cette inconnue, la valeur précédente.

Dans un grand nombre de cas, et spécialcment en astronomic, les élémens que l'on veut déterminer, sont déjà connus à fort peu prés, et n'ont besoin que de légères corrections que l'on cherche à obtenir par des observations nombreuses et précises. Pour cela, on regarde chaque obscrvation, comme une fonction des élémens. En substituant dans cette fonction, la valeur approchée de chaque élément, plus sa correction considérée comme une inconnue; en développant ensuite, la fonction, dans une série ordonnée par rapport aux puissances et aux produits de ces inconnues, et négligeant, vu lcur petitesse, les 
carrés et ces produits; enfin, en égalant la série, à l'observation diminuée de son erreur; on forme une équation du premier degré entre ces inconnues. C'est ce que l'on nomme équation de condiiton. On combine ensuite ces équations de condition, de manière à les réduire à un nombre d'équations finales, égal à celui des incormues. La résolution de ces équations donne les valeurs des inconnues, ou les corrections des divers élémens.

La manière la plus générale de former ces équations finales, consiste à multiplier chacune des équations de condition, par un facteur indéterminé : la somme de ces produils, en y supposant nul, tout ce qui cst relatif aux erreurs des observations, donnera une première équation finale. Un second système de facteurs donnera une seconde équation finale, et ainsi des autres. L'analyse des fonctions génératrices donne l'expression de l'erreur moyenne à craindre sur la correction de chaque élément, obtenue par la résolution de ces équations finales. Si l'on détermine les facteurs, par la condition que chacune de ces expressions soit un minimum; on trouve que le premier système de facteurs est formé des coefficiens de la première inconnue, dans chaque équation de condition; que le second système est formé des coefficiens de la secondo 
inconnue, etc.; d'ou il est facile de conclure queles corrections des ćlémens, les plus avantageuses, sont généralement, comme dans le cas d'une seule variable, celles que l'on obtient, lorsqu'on reud un minimum, la somme des carrés des erreurs de chaque observation, en y faisant varier successivement les corrections inconnues. Dans ce cas général, l'analyse doune l'expression de l'erreur moyenne à craiudre encore sur chaque élément; mais quoique très-simple, cette expression ne peut pas êlre comprise sans le sccours de l'algèbre.

Nous avons supposé forl grand, le nombre des observations; et la règle précédente est d'autant plus exacte, que ce nombre est plus considénable. Mais dans le cas même où il est petit, il paraît naturel d'employer la inême règle qui dans tous les cas, offire un moyen simple d'obırir sans tâlonmement, les correçions que I'on cherche à déterminer.

Cette régle peut servir encore à comparer la précision de diverses tables astronomiques d'an même astre. Ces tables peuvent toujours être supposées réduites a la même forme, ct alors elles ne different que par les époques, les moyens mouvemens, et les coefficiens des argumens; car si l'une d'elles contient un argument qui ne se trouve point dans les autres, il est clair que cela revient à supyoser 
nul dans celles-ci, le coefficient de cet argument. Maintenant, si l'on rectifiait ces tables, en les comparant à la tolalité des bonnes observations; elles satisferaient, par ce qui précède, à la condition que la somme des carrés des erreurs soit un minimum; les tables qui comparées à un nombre considérable diobservalions, approchent le plus, de cette condilion, méritent donc la préférence.

Des Tables de morlalité, et des durécs moyennes de la vie, des mariages el des associations quelconques.

La manicre de former les tables de mortalité, est trís-simple. On prend sur les registres des naissances et des morts, un grand nombre d'enfans que l'on suit pendant le cours de lcur vie, en déterminant combien il en reste à la fin de chaque année de leur âge, et l'on écrit ce nombre vis-a-vis de lannée finissante. Mais comme dans les deux premières annćes de la vic, la mortalité est trísrapide; il faut pour plus d'exactitude, indiquer dans ce premier âge, le nombre des survivaus à la lin de chaque demi-année.

Si l'on divise la somme des anníes de la vie de tous les individus inscrits dans une table de mortalicé, par le nombre de ces individus, et 
si de ce quotient, on soustrait une demi-amnée; on aura la durée moyenne de la vie, que l'on trouve ainsi de vingt-huit ans et demi a peu près. Cette soustraction ne doit avoir licu, que dans le cas où la table n'indique point le nombre des vivans à la fin de la première demi-annce : elle est fondée sur ce que la mortalité pouvant être supposée uniformément répandue sur la prenière annćc; la partic de la durée moyenne de la vie, correspondante à cette annéc, n'est que la moitié de celle qui aurait lieu, si la mort ne frappait les individus qu'à la fin de l'année. La durce moycune de ce qui reste encore à vivre, lorsqu'on est parvenu à un âge quclconque, se délermine en faisant une somme des années qu'ont vécu au-delì de cet âge, tous les individus qui l'ont atteint; en la divisant par le nombre de ces individus, et en retranchant une demi-année, de ce quotient. Ce n'est point au moment de la naissance, que la durée moyenne de la vie, cst la plus grande; c'est lorsqu'on a échappé aux dangers de la premicre enfance, et alors elle est d'environ quarante-trois ans. Lá probabilité d'arriver à un âge quclconque, en partant d'un âge donnô, est égale au rapport des deux nombres d'individus indiçués dans la table, à ces deux âges. 
La précision de ces résultats exige que pour la formation des tables, on emploie un très-grand nombre de naissances. L'analyse donne alors des formules très-simples pour apprécier la probabilité que les nombres indiqués dans ces tables ne s'écarteront de la vérité, que dans d'étroites limites. On voit par ces formules, que l'intervalle des limites diminue, et que la probubilité augmente, à mesure que l'on considére plus de naissances; ensorte que les tables représenteraient exactement la vraie loi de la mortalité, si le nombre des naissances employées devenait inlini.

Une table de mortalité est donc une table des probabilités de la vie humaine. Le rapport des individus inscrits à côté de chaque année, au nombre des naissances, est la probabilité qu'un nouveau-né atteindra celte année. Comme on estime la valeur de l'espérance, en faisant une somme des produits de chaque Lien espéré par la probabilité de l'obtenir; on peut également évaluer la durée moyenne de la vie, en ajoutant les produits de chaque année par la probabilité d'y arriver. Ainsi en formant une suite de fractions dont le dénominateur commun est le nombre des nouveau-nés de la table, et dont les numérateurs sont les nombres inscrits à côté de chaque 
année; la somme de toutes ces fractions scra la durée moyenne de la vie, dont il faut pour plus d'exactitude, retrancher une demi-année; ce qui conduit au même résultat ģıe la règie prócédente. Mais celte manicre d'envisager la durée moyenne de la vie, a l'avantage de faire voir que dans une population stationnaire, c'est-à-dire telle que le nombre des naissances égale celui des morts; la durée moyenne de la vie est le rapport méme de la population aux naissances annuelles; car la population élant supposée stationnaire, Ie nombre des individus d'un âge compris entre deux années consécutives de la table, est égal au nombre des naissances annuelles, multiplié par la demi-somme des probabilités d'atteindre ces années; la somme de tous ces produits sera donc la population entiere; or il est aisé de roir que cette somme divisće par le nombre des naissances annuelles, coïncide avec la durée moyenne de la vie, teile que nous venons de la dérinir.

Il est facile au moyen d'une table de mortalite, de former la table correspondante de la population supposée stationnaire. Pour cela, on prend des moyennes arithmétigues entre les nombres de la table de mortalité correspondans aux âges, zéro el un an, un et deux ans, deur et rois ans, olc. La somme do 


\section{SUR LES PROBABILITĹLS.}

toutes ces moyennes est la population entière: on l'écrit à côté de l'àge zéro. On retranche de cette somme, la première moyenne; et le restc est le nombre des individus d'un an et au-dessus : on l'écrit à còté de l'année .. On retranche de ce premier reste, la seconde moyenne; ce second reste est le nombre des individus de deux années, et au-dessus : on l'écrit à côté de l'année 2 ; ct ainsi de suite.

Tant de causes variables influent sur la mortalité, que lés tables qui la représentent, doivent changer suivant les lieux et les temps. Les divers états de la vie offrent à leur égard, des différences sensibles relatives aux fatigues ct aux dangers inséparables de chaque état, et doni il est indispensable de tenir compte dans les calculs fondés sur la durée de la vie. Mais ces différences n'ont pas encore été suffisamment observées. Elles le seront, un jour; alors on saura quel sacrifice de la vie, chaçue profession exige, et l'on profitera de ces connaissances, pour en diminuer les dangers.

La salubrité plus ou moins grande du sol, sa température, les moeurs des habitans, et les opérations des gouvernemens ont sur la mortalité, une influence considérable. Mais il faut toujours faire précéder la recherche de la cause des différences observées, par celle de la probabilité avec laquelle cette cause est 
indiquée. Ainsi le rapport de la population aux naissances annuelles, que l'on a vu s'élever en France, à vingt-huit et un tiers, n'est pas égal à vingt-cinq dans l'ancien duché de Milan. Ces rapports établis l'un et l'autre, sur un grand nombre de naissances, ne permettent pas de révoquer en doute, l'existence dans le Milanais, d'une cause spéciale de mortalité, qu'il importe au gouvernement de ce pays, de rechercher et de faire disparaitre.

Le rapport de la population aux naissances s'accroîtrait encore, si l'on parvenait à diminuer ou à éteindre quelques maladies dangereuses et très-répandues. C'est ce que l'on a fait heureusement pour la petite vérole, d'abord par l'inoculation de cette maladie; ensuite d'une maniere beaucoup plus avantageuse, par l'inoculation de la vaccine, découverte inestimable de Jenner qui par lì s'est rendu l'un des plus grands bienfuiteurs de l'humanité.

La petite vérole a cela de particulier, savoir que le même individu n'en est pas deux fois atteint, ou du moins, ce cas est si rare, que l'on peut en faire abstraction dans le calcul. Cette maladie à laquelle peu de monde échuppait avant la découverte de la vaccine, est souvent mortelle et fait pórir un septième de ceux qu'elle attaque. Quelquefois, elle est 
bénigne, et l'expérience a fait connaître qu'ou lui domait ce dernier caractère, en l'inoculant sur des personnes saines, préparées par un bon régime, et dans une saison favorable. Alors le rapport des individus qu'elle fait périr, aux inoculés, n'est pas un trois-centième. Ce grand avantage de l'inoculation, joint à ceux de ne point altérer la beauté, ct de préserver des suiles fächeuses que la petite vérole naturelle entrâne souvent après elle, la fit adopter par un grand nombre de personnes. Sa pratique fut vivement recommandée; mais, ce qui arrive presque toujours dans les choses sujettes i des inconvéniens, elle fut virement combattue. Au milieu de cette dispute, Daniel Bernoulli se proposa de soumettre au calcul des probabilités, l'influence de l'inoculation sur la durée moyenne de la vie. Manquant de données précises sur la mortalité produite par la pelite vérole, aux divers âges de la vic; il supposa que le danger d'avoir cette maladie et celui d'en périr, sont les mêmes à tout âge. Au moyen de ces suppositions, il parvint par une anilyse délicate, à convertir une table ordinaire de mortalité, dans celles qui auraient lieu, si la petite vérole n'existait pas, ou si elle ne faisait pirir qu'un trés-petit nombre de malades; et il en conclut que l'juoculation augmenterait de trois ans au 
moins, la durée moyenne de la vie; ce quí lui parut mettre hors de doute, l'avanlage de cette opération. D'Alembert attaqua l'analyse de Bernoulli, d'abord sur l'incertilude de ses deux hypothèses; ensuite, sur son insuffisance, en ce que l'on n'y faisait point entrer la comparaison du danger prochain quoique très-petit, de périr par l'inoculation, au danger beaucoup plus grand, mais plus éloigné, de succomber à la petite vérole naturelle. Celte considération qui disparait, lorsque l'on considere un grand nombre d'individus, est par là, indifférente aux gouvernemens, et laisse subsister pour eux, les avantages de l'inoculation; mais elie est d'un grand poids pour un père de famille qui doit craindre, en faisant inoculer ses cnfans, de voir bientôt périr ce qu'il a de plus cher au monde, et d'en être cause. Beaucoup de parcus ćtaient retenus par cette crainte, que la déconverte de la vaccine a heurcusement dissipće. Par un de ces mystères que la nature nous offre si fréquemment, le vaccin est un préservatif de la petite vérole, aussi sûr que le virus variolique, et il'n'a aucun danger : il n'expose à aucune maladie, et ne demande que trèspeu de soins. Aussi sa pratique s'est-elle promptement répandue, et pour la rendre unircrselle, il ne reste plus à vaincre que 
Pincrtie naturelle da peuple, contre laquelle il faut lutter sans cesse, même lorsqu'il s'agit de ses plus chers intérêts.

Le moyen le plus simple de calculer l'avantage que produirait l'extinction d'une maladie, consiste à déterminer par l'observation, le nombre d'individus d'un âge donné, qu'elle fait périr, chaque annéc, et à le retrancher du nombre des morts au même ige. Le rapport de la différence, au nombre tolal d'individas de l'âge donné, serait la probabilité de périr à cet âge, si la maladie n’existait pas. En laisant done une somme de ces probabilités depuis la naissance jusqu'à un âge quelconque, ct retranchant cette somme de l'unité; le reste sera la probabilité de vivre jusqu'à cet âge, correspondante à l'extinction de la maladie. La série de ces probabilités sera la table de mortalité, relative à cette hypothèse; et l'on en conclura par ce qui précède, la durée moyenne de la vie. C'est ainsi que Duvilard a trouvé l'accroissement de la durée moyenne de la vie, dú à l'inoculation de la vaccine, de trois ans au moins. Un accroissement aussi considérable en produirait un fort grand dans la population, si d'ailleurs, elle n'étail pas restreinte par la diminution relative des subsistances.

C'est principalement par le défuut des 
subsistances, que la marche progressive de la population est arrêtéc. Dans toutes les espèces d'animaux et de végétaux, la nature tend sans cesse à augmenter le nombre des individus, jusqu'à ce qu'ils soient au niveau des moyens de subsister. Dans l'espèce humaine, les causes morales ont une grande influence sur la population. Si le sol, par de faciles défrichemens, peut fournir une nourriture abondante à des générations nouvelles; la certitude de faire vivre une nombreuse famille, encourage les mariages, et les rend plus précoces et plus féconds. Sur un sol pareil, la population et les subsistances doivent croître à-la-fois en progression géométrique. Mais quand les défrichemens deviennent plus difficiles et plus rares; alors l'accroissement de la population diminue : elle se rapproche continuellement de l'état variable des subsistances, en faisant autour de lui, des oscillations, à peu près comme un pendule dont on proméne d'un mouvement retardé, le point de suspension, oscille autour de ce point, par sa pesanteur. Il est difficile d'évaluer le maximum d'accroissement de la population : il parait d'après quelques observations, que dans de favorables circonstances, la population de l'espèce humaine pourrait doubler, tous les quinze ans. On cstime que dans 
I'Amérique septentrionale, la période de ce doublement est de vingt-cinq années. Dans cet ćtat de choses, la population, les naissances, les mariages, la morlalité, tout croît suivant la même progression géométrique dont on a le rapport constant des termes consécutifs, par l'olsscrvation des naissances annuelles à deux époques.

Une table de mortalité, représentant les probabilités de la vie humaine; on peut déterminer à sou moyen, la durée des mariages. Supposons pour simplifier, que la mortalité soit la mêtne pour les deux sexes; on aura la probabilité que le mariage subsistera un an, ou deux, ou trois, etc.; en formant une suite de fractions dont le dénominateur commun, est le produit des deux nombres de la table, correspondans aux âges des conjoints, et dont les numérateurs sont les produits successifs des nombres correspondans à ces âges augmentés d'une année, de deux, de trois, etc. La somme de ces fractions, augmentée d'un demi, scra la durće moyenne du mariage, l'annéc étant prise pour unité. Il est facile d'étendre la mềme règle, à la durée moyenne d'une association formée de trois ou d'un plus grand nombre d'individus. 
Des bénéfices el des établissemens qui dépendent de la probabilité desévenemens.

Rappelons ici ce que nous avons dit en parlant de l'espérance. On a vu que pour avoir l'avantage qui résulte de plusieurs événemens simples, dont les uns produisent un bien, et les autres une perte; il fiut ajouter les produits de la probabilité de chaque événement favorable, par le bien qu'il procure, et retrancher de leur somme, celle des produits de la probabilité de chaque événement défavorable, par la perte qui y est altachée. Mais quel que soit l'avantage exprimć par la différence de ces sommes, un seul événement composé de ces événemens simples, ne garantit point de la crainte d'ćprouver une perle réclle. On conçoit que cetie crainte doit diminuer lorsque l'on multiplic lévénement composé. L'analyse des probabilités condui: il ce théorème général.

Par la répétition d'un événement avantageux, simple on composé, le bénéfice réel devient de plus en plus probable, et s'accroit sans cesse : il devient certain, dans lhypothèse d'un nombre infini de répélitions; et en le divisant par ce nombre, le quolient ou le bénélice moyen de chaque événenent, esł 


\section{SUR LES PROBABLLITÉS.}

l'espérance mathématique elle-même, ou l'avantage relatif à l'événement. Il en est de même de la perte qui devient certaine à la longne, pour peu que l'événement soit désavantageux.

Ce théorème sur les bénéfices ét les pertes, est analogue à ccux que nous avons donnés précédemmení sur les rapports qu'indique la répélition indéfnic des événemens simples ou composés; et comme eux, il proure que la régularilé finit par s'élabjir dans les choses même, les plus subordonnćes à ce que nous nommons hasard.

Lorsque les événemons sont en grand nombre, l'analyse dunne encore une expression fort simple de la probabilité que le bénéíce réel sera compris dans des limiles déterminées, expression qui rentre dans la loi générale de la probalilité, que notis avons domée ci-dessus, en parlant des probabilités qui résultcnt de la multiplication indéínie des événemens.

C'est de la vérité du théorème précédent, que dépend la stabilité des établissemens fondés sur les probabilités. Mais pour qu'il puisse leur être appliqué, il faut que ces établissemens, par de nombreuses aflaires, multiplient les événemens avantageux.

On a fondé sur les probabilités de la vie 
humaine, divers établissemens, tels que lest rentes viagères et les tontines. La méthode la plus générale et la plus simple de calculer les bénéfices et les charges de ces établissemens, consiste à les réduire en capitaux actuels. L'intérêt annuel de l'unité, est ce que l'on nomme taux de l'inlérêt. A la fin de chaque année, un capital acquiert pour facteur, l'unité plus le tuux de l'intérêt; il croìt donc suivant une progression géométrique dont ce facteur est la raison. Ainsi par l'effet du temps, il devient immense. Si, par cxemple, le taux de l'intérêt est $\frac{1}{20}$ ou de cinq pour cent; le capital double à fort peu près cn quatorze ans, quadruple en vingt-neuf ans, et dans moins de trois siecles, il devicnt deux millions de fois plus considéruble.

Un accroissement aussi prodigieux a fait naîlre l'idée de s'en servir, pour amortir la dette publique. Si l'on crée un premier fonds damortissement que l'on place sans cesse avec les intérêts, sur les effets publics, en profitant surtout des momens de baisse; et si, lorsque les besoins de l'élat ubligentà faire des emprunts, on en consacre une partie, ì l'accroissement du fonds d'amortissement; il est visible que ces opérations auront le double avantage d'accroitre ce fonds, et de soutenir le crédit el les effets publics; cl qu’à la longue, 
Ia caisse d'amortissement absorbera une grande paric de la dette nationale. D'heureuses experiences ont pleinement confirmó ces avantages. Mais la fidélité dans les engagemens et la stabilité, si nécessaires au succès de pareils étabilissemens, ne peuvent être bien garanties, que par un gouvernement représenlatif.

11 resulte de ce qui précéde, que le capilal actuel équivalent à une somme qui ne doit être payće qu'aprćs un cortain nombre d'années, est ćgal à celte somme multiplićc par la probabilité quielle sera payée à cette époque, ct divisée par I'unité augmentée du tiux de l'intérêt, ćlevée à une puissance cxprince par le nombre de ces années.

Il est facile d'appliquer ce principe, aux rentes viagires sur unc ou plusieurs tèles, ct aux caisses d'épargne et d'assurance d'une nature quelconque. Supposons que l'on se propose de former unc lable de rentes viagires, d'aprés une table donnée de mortalité. Une rente viagere payable au bout de cing ans, par cxemple, et réduite en capilal actuel, est par ce principe, égale au produit des deux quantilés suivantes, savoir, la rente divisée par la cinquième puissance de l'unité augmentée du taux de lintérêt, et la probabilité de la payer. Cette probabilité est le rapport 
150

inverse du nombre des individus inscrits dans

la table, vis-à-vis de l'âge de celui qui constitue la rente, au nombre inscrit vis-a-vis de cet âge augmenté de cinq années. En formant done une suite de fractions dont les dénominatcurs sont les produits du nombre de personues indiquées dans la table de mortalité, comme vivantes à l'àge do celui qui constitue la rente, par les puissances successives de l'unité augmentée du taux de l'intérêt, et dont les numérateurs sont les produits do la rente, par le nombre des personnes vivantes au micme âge augmenté successivemenl d'une annće, de deux années, etc., la somme de ces fractions sera le capital requis pour la renle viagère à cet îge.

Supposons maintenant qu'une personne veuille, au moyen d'unc rente viagère, assurer à ses héritiers, un capital payable à la fin de l'année de sa mort. Pour déterminer la valeur de cette rente, on peut imaginer gue la personne cmprunle cn viager à une caisse, ce capital divisé par l'unité augmentce du taux de lintérêt, et qu’clle le place à intérèt perpétucl à la :nême caisse. Il est clair que ce capital sera dû par la caisse, à ses héritiers, à la fin de l'annéc de sa mort; mais elle n'aura payé, chaque annéc, que l'excès de l'intérêt viager sur l'intérêt perpétuel. La table 
des rentes viagères fait donc connaitre ce que la personne doit payer annuellement à la caisse, pour assurer ce capital après sa mort.

Les assurances maritimes, celles contre les incendies et les orages, et généralement tous les établissemens de ce genre, se calculent par les mêmes principes. Un négociant a des vaisscaux en mer, il veut assurer lenr valeur et celle de leur cargaison, contre les dangers qu'ils peuvent courir : pour cela il donne une somme à une compagnie, qui lui répond de la valeur estimée de ses cargaisons etde ses vaisseaux. Le rapport de cette valeur à la somme qui doit être donnće pour prix de lassurance, dépend des dangers auxquels les vaisseaux sont exposés, et ne peut être apprécié que par des observations nombreuses sur le sort des vaisscaux partis du port pour la même destination.

Si l'assureur ne donnait à la compagnie d'assurance, que la somme indiquée par le calcul des probabilités, cette compagnie ne pourrait pas subvenir aux dépenses de son établissement; il fuut donc qu'il paie d'une somme plus forte, le prix de son assurance. Mais alors quel est son avantage? C'est ici que la considération de l'espérance morale devient nécessaire. On conçoit que le jeu le plus égal devenaut, conme on l'a vu précédemment, 
désavantageux, parce qu'il ćchange une mise certaine, conlre un bénéfice incertain; l'assurance par laquelle on échange l'incertain contre le certain, doit être avantageuse. C'est en eflet, ce qui résulte de la règle que nous avons donnéc ci-dessus pour déterminer l'espérance morale, et par laquelle on voit de plus jusqu'où peut s'étendre le sacrifice que l'on doit faire à la compagnic d'assurance, en conservant toujours un avantage moral. Celte compagnie peut done en procurant cel avantage, faire elle-même un grand bénéfice, si le nombre des assureurs est très-considérable, condition nécescaire à son existence durable. Alors son bénéfice devient cerlain, et ses espérances mathématique et morale coïncident. Car l'analysc conduit à ce théorème général, savoir, que si les expectatives sont très-nombreuses, les denx espérances approchent sans cesse l'une de l'autre, et finissent par coïncider dans le cas d'un nombre inlini d'expectatives.

Parmi les établissemens fondés sur les probabilités de la vie humaine, les plus utiles sont ceux dans lesquels, au moyen d'un léger sacrifice de son revenu, on assure l'existence de sa famille pour un temps ou l'on doit craindre de ne plus suffire à ses besoins. Autant le jeu estimmoral, autant ces élablissemens sont 


\section{SUR IES PROBABILITLS.}

avanlageux aux moeurs, en favorisant les plus doux penchans de la nature. Le Gouvernement doil donc les encourager et les respecter dans ses vicissitudes; car les espérances qu'ils présentent, portant sur un avenir éloigné, ils ne peuvent prospérer qu'a l'abri de toute inquiétude sur leur durée.

Disons un mot des emprunts. Il est clair que pour emprunter en perpétuel, il faut payer, chaque année, le produit du capital par le taux de l'intérêt. Mais on peut vouloir acquilter ce capilal, en paiemens égaux fuits pendant un nombre déterminé d'années, paicmens que l'on nomme annuités, et dont on obtiont ainsi la valeur. Chaque annuité, pour être ríduile au moment actuel, doit être divisée par l'unité augmentée du laux de l'intérêt, et élevéc à une puissance égalc au nombre des années après lesquelles on doit payer celle annuité. En formant donc une progression géométrique dont le premier terme est l'annuité divisée par l'unilé augmentée du taux de l'intérêt, et dont le dernier est cette annuilé divisée par la mềne quantité élevée à une puissance égale au nombre des années pendant lesquelles le paiement doit avoir lieu; la somme de cette progression sera équivaIente au capilal cmprunté; ce qui détermine la valeur de l'annuité. Si l'on veut faire un 
emprunt viager; on observera que les tables de rentes viagères domnant le cerpital requis pour constituer une rente viagère, à un âge quelconque; une simple proportion donnera la rente que l'on doit faire a l'individu dont on cmprunte un capital. On peut calculer par ces principes, tous les modes possibles d'emprunt.

Des illusions dans l'estimalion des probabililés.

L'esprit a ses illusions, comme le sens de la vue; et de même que le toucher rectifie celles-ci, la réflexion et le calcul corrigent également les premières. La probabilité fondée sur une expérience journalière, ou exagéréc par la crainte et l'espérance, nous frappe plus qu'une probabilité supérieure, mais qui n'est qu'un simple résultat du caleul. $\Lambda$ insi nous ne craignons point pour de faibles avantlages, d'exposer notre vie, à des dangers beaucoup moins invraisemblables que la sortie d'un quine à la loterie de France; et cependant personne ne voudrait se procurer les mêmes avantages, avec la certitude de perdre la vic, si ce quine arrivait.

Nos passions, nos préjugés, et les opinions. dominantes, en exagérant les probubilités qui 
leur sont favorables, ct en alténuant les probabilités contraires, sont des sources abondantes d'illusions dangereuses.

Les maux présens et la cause qui les fait naitre, nous affectent beaucoup plus, que le souvenir des maux produits par la cause contraire, et nous empêchent d'apprécier avec justesse, la probabilité des moyens propres à nous préserver des uns el des autres. C'est ce qui porte alternativement vers le despotisme et vers l'anarchic, les pcuples sortis de l'état du repos, dans lequel ils ne rentrent jamais qu'apris de longues et cruelles agititions.

Cette impression vive que nous recevons de la présence des événemens, et qui nous laisse à peine remarquer lcs événemens contraires observés par d'autres, est une cause principale d'erreurs, dont on ne peut trop se garantir. Nous croyons voir, par exemple, avec évidence, la vérité d'un fait attesté par des hommes dont nous avons souvent reconnu la véracité, surtout lorsqu'il est accompagné de circonstances qui viennent à l'appui de leur témoignage, et que nous avous pris soin de vérifier nous-mêmes. Accoutumés à nous conduire d'après de semblables preuves, et n'ayant jamais été trompés par elles, nous les regardons comme infaillijles; et s'il s'agit d'un 
dćlit, nous ne balançons point à condamner lindividu qu'elles inculpent. Cependant, les récils des Causes célèbres suffiscnt pour nous convaincre que les prcuves morales les plus fortes sont toujours susceptibles d'crreurs. Nous devrions done alors nous abstenir de juger. Mais si les preuves sont telles que les inconvéniens de l'erreur à craindre, multipliés par sa petite probabilité, donnent un produit très-inférieur au danger qui résulterait de l'impunité du crime; le jugement est commandé par l'intérêt de la société : quelquefois même, dans un danger imminent, cet interêt exige que le magistrat se relâche des formes sagementétablies pour la sûreté de l'innocence.

Les coïncidences de quelques événemens remarquables, avec les prédictions des astrologues, des devins et des augures, avec les songes, avec les nombres et les jours répulés heureux ou malhcureux, ctc., ont domné naissance à une foule de préjugés encore trèsrépandus. On ne rélléchit pas au grand nombre de non-coïncidences qui n'ont fait aucune impression, ou que l'on ignore. Cependant, c'est le rapport seul des unes aux autres, qui peut donner la probabilité des causes auxquelles on attribue les coincidences. Si ce rupport était connu, l'expérience confirmerait sans doute, ce que le bon sens et la raison 
nous dictent à l'égard de ces préjugés. Ainsi lc philosophe de l'antiquité, auquel on montrait dans un temple, pour exalter la puissance du dieu qu'on y adorait, les ex-yoto de tous ceux qui aprèsl'avoir invoqué, s'étaient sauvés du naufrage, faisait une question conforme au calcul des probabilités, en demandant combien de personnes, malgré cette invocation, avaient péri.

C'est principalement au jeu, qu'une foule d'illusions entretient l'espérance, et la soutient contre les chances défavorables. La plupart de ceux qui mettent atix loteries, ne savent pas combien de chances sont à leur avantage, combien leur sont contraires. Ils n'envisagent que la possibilité, pour une mise légère, de gagner une somme considérable; et les projets que lcur imagination enfante, exagerent a leurs yeux, la probabilité de l'obtenir. Ils seraient sans doute, effrayés du nombre immense des mises perdues, s'ils pouvaient les connaitre; mais on prend soin au contraire, de donner aux gains, une grande publicité.

Lorsqu'a la loteric de France, un numéro n'est pas sorti depuis long-temps; la foule s'empresse de le couvrir de mises. Elle juge que le numéro resté long-temps sans sortir, doit an prochain tirage, sortir de préférence aux autres. Une erreur aussi commune me 
paraît tenir à une illusion, par lacuuclle on se reporte involontairement ì. l'origine des événemens. Il est, per exemple, très-peu vraisemblable qu'au jeu de croix et pile, on amènera croix, dix fois de suite. Cette invraisemblance qui nous frappe encore, lorsqu'il est arrivé neuf fois, nous porte à croire qu'au dixième cotip, pile arrivera. Cependant loin de nous faire juger ainsi; le passé, en indi-quant dans la pièce, une plus grande pente pour croix que pour pile, rend le premier de ces événemens, plus probáble que l'autre: il augmente, comme on l'a vu, la probabilité d'amener croix au coup suivant. Une illusion semblable persuade a beaucoup do monde, que l'on peut gagner sùrement ì la loterie, cn plaçant chaque fois, sur un mème numéro jusqu'à sa sortie, une mise dont le produit surpasse la somme de toutes les mises. Mais quand même de semblables spéculations ne seraicnt. pas souvent arrêtées par limpossibilité de les soutenir; elles ne diminueraient point le désavantage mathématique des spéculateurs, et clles accroitraient leur dísavantage moral; puisqu'à chaque tirage, ils exposcraient une plus grande partie de leur fortune.

Par une illusion contraire aux précédentes, on cherche dans les tirages passés, les numéros le plus souvent sortis, pour en former 
des combinaisous sur lesquelles on croit placer sa mise avec avantage. Mais vu la manière dont lc méiange des numéros se fait à la loterie; le passé ne doit avoir sur l'avenir, aucune influence. Les sorties plus fréquentes d'un numéro ne sont que des ariomalies du hasard : j'en ai soumis p'usieurs au calcul, et j'ai conslamment trouvé qu'elles étaient renfermées dans les limites que la supposition d'une égale possibilité de sortie de tous les numéros, permet d'admettre sans invraisemblance.

Dans une longue séric d'événemens du même genre, les seules chances du hasard doivent quclquefois offirir ces veines singuličres de bonheur ou de malleur, que la plupart des joucurs ne manquent pas d'attribuer à une sorte de fatalité. 11 arrive souvent dans les jeux qui dépendent a-la-fois du hasard et de l'habileté des joucurs, que celui qui perd, troublé par sa perle, cherche à la réparer par des coups hasardeux quil éviterait dans une autre situation: il aggrave ainsi son propre malheur, et il en prolonge la durée. C'est cependant alors, que la prudence devient néces. siire, et qu'il importe de se convaincre que le désavantage moral attaché aux chances défavorables, s'accroît par le malheur même. Le sentiment par lequel l'homme s'est placé 
long-temps, au centre de l'univers, on se considérant comme l'objet spécial des soins de la nature, porte chaque individu à se faire le centre d'une sphcre plus ou moins étendue, et à croire que le hasard a pour lui des préférences. Soutenus par celte opinion, los joueurs exposent souvent des sommes considérables, à des jeux dont ils savent que les chances leur sont contraires. Dans la conduite de la vie, une semblable opinion peut quelquefois avoir des avantages; mais le plus souvent, elle conduit a des entreprises périlleuses et funestes. Ici, comme en tout, les illusions sont dangereuses, et la vérilé seule est généralement utile.

Un des grands avantages du calcul des probabilités, est d'apprendre à se délier des premiers aperçus. Comme on reconnaît qu'ils trompent souvent, lorsqu'on peut les soumettre au calcul; on doit en conclure que sur d'autres objets, il ne faut s'y livrer qu'avec une circonspection extrême. Prouvons cela par des exemples.

Une urne renferme quatre boules noires ou blanches, mais qui ne sont pas toutes de la même couleur. On a exirait une de ces boules, dont la couleur est blanche, et que l'on a remise dans l'urne pour procéder cucore a de semblables tirages. On demande la 
probabilité de n'extraire que des boules noires, dans les quatre tirages suivans.

Si les boules blanches et noires étaient en nombre égal, cette probabilité serait la qua. trième puissance de la probabilité $\frac{1}{2}$ d'extraire une boule noire à chaque tirage; elle serait donc $\frac{1}{16}$. Mais l'extraction d'une boule blanche an premier tirage, indique une supériorité dans le nombre des boules blanches de l'urne; car si l'on suppose dans l'urne, trois boules blanches et une noire, la probabilité d'en extraire une boule blanche est $\frac{3}{4}$; elle est $\frac{2}{4}$, si l'on suppose deux boules blanches et deux noires; enlin, elle se réduit à $\frac{1}{4}$, si lon suppose trois boules noires et une blanche. Suivant le principe de la probabilité des causes, tiréc des événemens, les probabilités de ces trois suppositions sont entre elles, comme les guantilés $\frac{3}{4}, \frac{2}{4}, \frac{1}{4}$; elles sont par conséquent égales à $\frac{3}{6}, \frac{2}{6}, \frac{1}{6}$. Il y a ainsi ciuq contre un a parier que le nombre des boules noires est inféricur, ou tout au plus égal à celui des blanches. Il semble donc que d'après l'extraction d'une boule blanche au premier tirage, la probabilité d'extraire de suite quatre boules noires, doit être muindre que dans le cas de l'égalité des couleurs, ou plus petite qu'un seizième. Cependant cela n'est pas, et l'on trouve par un calcul fort simple, cette probabilité 
plus grande qu'un quatorzieme. En effet, elle scruit la quatrième puissance de $\frac{3}{4}$, de $\frac{2}{4}$ et de $\frac{x}{4}$, dans la première, la seconde et la troisième des suppositions précédentes sur les couleurs des boules de l'urne. En multipliant respectivement chaque puissance, par la probabilité de la supposition correspondante, ou par $\frac{3}{6}$, $\frac{2}{6}$ et $\frac{1}{6}$; la somme des produits sera la probabilité d'extraire de suite, quatre boules noires. On a ainsi pour cette probabilité, $\frac{29}{384}$, fraction moindre que $\frac{1}{14}$. Ce paradoxe s'explique en considérant que lindication de la superiorité des boules blanches sur les noires, par le premier tirage, n'exclut point la supériorité des boules noires sur les blanches, supériorité qu'exclut la supposition de l'égalité des couleurs. Or cette supériorité quoique peu vraisemblable, doit rendre la probabilité d'amener de suite, un nombre donné de boules noires, plus grande que dans celte supposition, si ce nombre est considérable; et lon vient de voir que cela commence, lorsque le nombre donné est égal à quatre.

Considérons encore une urne qui renferme plusicurs boules blanches et noires. Supposons d'abord qu'il n'y ait qu'une boule blanche et une noire. On peut alors parier avec égalité, d'extraire une boule blanche, dans un tirage. Mais si l'urne renferme trois boules dont 
deux soient ñoires; il semble que pour l'égalité du pari, on doit donner deux tirages à celui qui parie d'extraire la boule blancle: on doit endonner trois, si l'urne renferme trois boules noires et une blanche, et aiusi du reste; ensorte que pour compenser par le nombre des tirages, l'inégalité des chamees, ii faut donner aulant de tirages qu'il y a de chances contraires: on suppose toujours quaprès cliaque tirage, la boule extraite esi remise dans l'urne. Mais il est facile de se convaincre que ce premier aperçu est erroné. En effet, dans le cas de deux boules noires sur une blanche, la probabilité d'extraire de l'urne, deux boules noires en deux tirages, est la seconde puissance de $\frac{2}{3}$ ou $\frac{4}{9}$; mais cette probabilite ajoutée ì celle d'amener une boule blanche en deux tirages, est la certitude on l'unité; puisqu'il est certain que l'on doit amener deux boules noires, ou au moins une boule blanche; la probabilité de ce dernicr cas est donc $\frac{5}{9}$ fraction plus grande que $\frac{t}{2}$. Il y aurait plus d'avantage encore a parier d'amener une boule blanche en cinq tirages, lorsque l'urne conient cinq boules noires et une blanche; ce pari est même avantageux en quatre tirages: il revient alors à celui d'amener six en quatre coups, avec un seul dé.

Le chevalier de Meré, ami de Pascal, et 
qui fil naître le calcul des probabilités, en excitant ce grand géomètre à s'en occuper, lui disait « qu’il avait trouvé fausseté dans les ) nombres par cetle raison. Si l'on entre3) prend de faire six avec un dé, il y a de ) l'avantage àl'cntreprendre en quatre coups, ) comme de 671 à 625 . Si l'on cutreprend ) de laire sonnés avec deux dés, il y a dé》) savantage à l'entreprendre en 24 coups. ) Néaumoins 24 est à 36 nombre de faces de ) deux dés, comme 4 est à 6 nombre des ग) faces d'un dé. Voilá, écrivait Pascal à Fer2) mat, quel était son grand scandáe, qui lui ) faisait dire hautement, que les propositions \#n'ctaient pas constantes et que l'arithmé3) tiçue se démentail.... 11 a très-bon esprit; ) mais il n'est pas géométre : c'est, comme 》) vous savez, un grand délaut. ) Le chevalier de Meré trompé par une fausse analogie, pensait que dans le cas de l'égalité des paris, le nombre des coups doit croître proportionnellement au nombre de toutes les chances possibles, ce qui n'est pas exact, mais ce qui approche d'autant plus de l'être, que ce nombre est plus grand.

Je mets encore au rang des illusions, l'application que Leibnitz et Danicl Bernoulli ont faite du calcul des probabilités, à la sommatiou des séries. Si l'on réduit la fraction dout le 
numérateur est l'unité, et dont le dénominateur est l'unitié plus une variable, dans une snite ordonnée par rapport aux puissances de celte variable; il est facile de voir qu'en supposant la variable égale à l'unité, la fruction devient $\frac{1}{2}$, el la suite devient, plus un, moins un, plus un, moins un, etc. En ajoutant les deux premiers termes, les deux suivans, et ainsi du reste, on transforme la suite dans une autre dont chaque terme cst zćro. Grandi, jésuile italien, en avait conclu la possibilité de la création; parce que la suite étant toujours égale à $\frac{1}{2}$, il voyait cette fraction naître d'une infinité de zéros, ou du néant. Ce fut ainsi que Leibnitz crut voir l'image de la création, dans son Arithmétique binaire où il n'employait que les deux caractères zéro et l'unité. Il imagina que l'unité pouvait représenter Dicu; et zéro, le néant; et que l'Étre Suprême avait tiré du néant, tous les êtres, comme l'unité avec le zéro, exprime tous les nombres dans ce systéme. Cette idée plut tellement à Leibnitz, gu'il en fit part au jésuite Grimaidi, président du tribunal des mathématiques à la Chine, dans l'espérance que cet emblême de la création conver tirait au christianisme, l'empereur d'alors qui aimait purticulièrement les sciences. Je ne rapporte ce trait, que pour montrer 
jusqu'à quel point les préjugés de l'enfunce peuvent égarer les plus grands hommes.

Leibnitz toujours conduit par une métaphysique singulière el très-déliće, considéra que la suite, plus un, moins un, plus un, etc. devient l'unité ou zéro, suivant que l'on s'arrête à un nombre de termes, impair ou pair; et comme dans l'infini, il n'y a aucune raison de préférer le nombre pair ì l'impair, on doil, suivant les regles des probabilités, prendre la moitié des résultats relatif́s a ces deux espèces de nombres, et qui sont zéro et l'unité; ce qui donne $\frac{1}{2}$ pour la valeur de la série. Daniel Bernoulii a étendu depuis, ce raisonnement a la sommation des séries formées de termes périodiques. Mais toutes ces sériesn'ontpoint, a proprement parler, de valeurs : elles rien prennent que dans le cas oi leurs termes sont multipliés par les puissances successives d'une variable moindre que l'unité. Alors, ces séries sont toujours convergentes, quelque petite que l'on suppose la différence de la variable à l'unité; et il est facile de démontrer que les valeurs assignées par Bernoulli, en vertu de la règle des probabilités, sont les valeurs mêmes des fractions génératrices des súries, lorsque l'on suppose dans ces fractions, la variable égale à l'unité. Ces valeurs sont encore les limites dont les séries approchent 
de plus en plus, à mesure que la variable approche de l'unité. Mais lorsque la variable cst exactement égale àl'unité, les séries cessent d'être convergentes : elles n'ont de valeurs, qu'autant qu'on les arrête. La coincidence remarquable de cette application du calcul des probabilités, avec les limites des valeurs des séries périodiques, suppose queles termes de ces séries sont multipliés par toutes les puissances consécuitives de la variable. Mais ces séries peuvent résulter du développement d'une infinité de fractions différentes, dans lesquelles cela n’a pas lieu. Ainsi la série, plus un, moins un, plus un, etc. peut naitre du développenent d'une fraction dont le numératcur est l'unité plus la variabie, el dont le dénominateur est ce numérateur augmenté du carré de la variabie. En supposant la variable égale à l'unité, ce développement se change dans la sćrie proposée, et la fraction génératrice devient égale a $\frac{2}{3}$; les règles des probabilités domneraient done alors un faux résultat; ce qui prouve combien il scrait dingereux d'employer de semblables raisonnemens, surtout dans les sciences mathématiques, que la rigueur de leurs procédés doit éminemment distinguer. 


\section{Des divers moyens d'approcher de la cerlitude.}

L'induction, l'analogie, des hypothéses fondées sur les faits et rectifiées sans cesse par de nouvelles obscrvations, un tact heureux donmé par la nature et fortifié par des comparaisons nombreuses de ses indications avec l'expérience; tels son les principaux moyens de parvenir ì la vérité.

Si l'on considére avec attention, la série des objets de même nature; on aperçoit entre eux et dans leurs changemens, des rapports et rles lois qui se manifestent de plus en plus, à mesure que la série se prolonge, et qui, en s'étendant et se généralisant sans cesse, conduisent enfin au principe dont ils dépendent. Mais souvent ces lois el ces rapports sont enveloppés de tant de circonstances étrangères, qu'il faut une grande sagacité pour les démêler, et pour remonter ì ce principe: c'st en cela que consiste le véritable génie des sciences. L'analyse et la philosoplie naturelle doivent leurs plus importantes dicouvertes, à ce moyen fécond que l'on nomme induction. New ton lui a été redevable de son théorème du binome, et du principe de la gravitation universelle. Il est difficile d'apprécicr 
la probabilité de ses résultats. Elle se fondo sur ce que les rapports et les lois les plus simples, sout les plus communs : c'est ce qui se vérifie dans les formules de l'analyse, et ce que l'on retrouve dans les phénomènes naturels, dans la cristallisation, et dans les combinaisons chimiques. Cette simplicité de lois et de rapports ne paraitra point étonnante, si l'on considére que tous les effels de la nature, ne sont que les résultats mathématiques d'un petit nombre de lois immuables.

Cependant linduction, en fiisant découvrir les principes généraux des sciences, ne suffit pas pour les établir en rigucur. Il faut tonjours les confirmer par des démonstrations, ou par des expériences décisives; car thistuire des sciences nous montre que l'induction a quelquefois conduit a des résultats incxacts. Je citerai pour exemple, un théorime de Fermat sur les nombres premiers. Ce grand géometre qui avait profondément médité sur leur théorie, cherchait une formule qui ne renfermant que des nombres premicrs, donnât directement un nombre premier plus grand qu'aucun nombre assignable. L'induction le conduisit à penser que doux élevéà une puissance qui ćtait clle-même une puissance de deux, formait avec l'unité, un nombre premier. Ainsi deux élevé au carré, 
plus un, forme le nombre premier cinq : deux élevé à la seconde puissance de deux, ou seize forme avec un, le nombre premier dixsept. Il trouva que cola était encore vrai pour la huitième et la seizième puissance de deux, augmentée de l'unité; et cette induction appuyée de plusieurs considéralions arithmétiques, lui fit regarder ce résultat, comme général. Cependant il avoue qu'il ne l'avait pas encore démontré. En effet, Euler a reconnu que cela cesse d'avoir lieu pour la trentedeuxième puissance de deux, qui angmentéc de l'unité, donne 4294967297 , nombre divisible par 641.

Le chancelier Bacon, promoteur si éloquent de la vraie méthode philosophique, a fait de l'induction, un abus bien étrange, pour prouver l'immobilité de la terre. Voici comme il raisonne dans le Novum Organum, son plus bel ouvrage. Le mouvement des astres, d'orient en occident, est d'autant plus prompt, qu'ils sont plus éloignés de la terre. Ce mouvement est le plus rapide pour les ćtoiles : il so ralentit un peu pour Saturne, un peu plus pour Jupiter, et ainsi de suite, jusqu'a la lune et aux comètes les moins élevées. Il est encore perceptible dans. l'atmosphère, surtout entre les tropiques, à cause des grands cercles que les molécules de l'air y décrivent; enfin 


\section{SUR IES PROBABILITÉS.}

il est presqu'insensible pour locéan; il est donc nul pour la terre. Mais cette induction prouve seulement que les astres ont des mouvemens propres, contraires au muvvement réel on apparent qui emporte toute la sphère céleste d'orient en occident, et que ces mouvemens paraissent plus lents pour les astres plus éloignés; ce rui est conforme aux lois de l'optique. Bacon aurait dû être frappé de l'inconcevable vitesse qu'il faut supposer aux astres pour accomplir leur révolution diurne dans l'hypothèse de la terre immobile, et de l'extrême simplicité avec laquelle sa rolation explique comment des corps aussi distans les uns des autres, que les étoiles et les planètes, semblent tous assujétis à cettc révolution. Quant à l'Océan et à latmosphère, il ne devait point assimiler leur mouvement à celui des astres, qui sont détachés de la terre; au lieu que l'air et la mer faisant partie du globe terrestre, ils doivent participer à son mouvement ou a son repos. Il est singulier que Bacon porté aux plus grandes vues, par son génie, n’ait point été entrainé par l'idée majestueuse que le systéme de Copernic olfre de l'univers. II pouvait cependent trouver en faveur de ce système, de fortes analogies, dans les découvertes de Galilée, qui lui ćtaient connues. Il a donné pour la recherche de la 
vérité, le précepte, et non l'excmple. Mais en insistant avec toute la force de la raison et de l'éloquence, sur la nécessité d'abandonner les subtilités insignifiantes de l'école, pour se livrer aux observations et aux expériences, et en indiquant la vraie méthode de s'élever aux causes génćrales des phénomènes; ce grand philosophe a contribué aux progrès immenses que l'esprit humain a faits dans le beau siècle où il a terminé sa carrière

L'analogie est fondée sur la probabilité que les choses semblables ont des causes du même genre, et produisent les mêmes effets.'Plus la similitude est parfaite, plus grande est celte probabilité. Ainsi nous jugeons sans aucun doute, que des êtres pourvus des mêmes organes, exécutant les mêmes choses, et communiquant ensemble, éprouvent les mêmes sensations, et sont mus par les mêmes desirs. La probabilité que les animaux qui se rapprochent de nous par leurs organes, onl des sensations analogues aux nôtres, quoiqu'un peu inférieure a' celle qui est relative aux individus de notre espèce, est encore excessivement grande; et il a fallu toute linfluence des préjugés religieux, pour faire penser à quelques philosophes, que les animaux sont de purs automates. La probabilité de l'existence du sentiment décroit, à mesure quo la. 
similitude des organes avec les notres, diminue; mais clle est toujours très-forte, même pour les insectes. En voy ant ceux d'une même espece, exćcuter des choses fort compliquécs, exactement de la même manic̀re, de générations ea générations, et sans les avoir apprises; on est porlé à croire qu'ils agissent par une sorte d'allinilé, analogue à celle qui rapproche les molćcules des cristaux, mais qui se mêlant au sentiment altaché à toute organisation animale, produit avec la régularité des combinaisons chimiques, des combinaisons beaucoup plus singulicres : on pourraitpeul-ĉtre, nommer affinzié animale, ce mólange des allinilés ćlectives et du sentiment. Quoiqu'il existe beaucoup d'analogie entre lorganisation des plantes et celle des animaux; clle ne me paraît pas cependant sullisante pour élendre aux végćtaux, la fircullé de sentir; comme rien n’autorise à la leur reluser.

Le solcil faisanl éclore par l'action bienfiisante de sa lumière et de sa chaleur, les animaux et les plantes qui couvrent la terre; nous jugeons par l'analogie, qu'il produit des eflels semblables sur les autres plancles; car il n'est pas naturel de penser que la matière dont nous voyous l'aclivité se développer en tant de façons, est stérile sur une aussi grosse 
planc̀te que Jupiler qui, comme le globe terrestre, a ses jours, ses nuits et ses années, et sur lequel les observations indiquent des changemens qui supposent des forces trésactives. Cependant ce serait donner trop d'extension à l'analogie, que d'en conclure la similitude des habitans des plancites, et de la terre. L'bomme fait pour la température dont il jouit, et pour l'élément qu'il respire, ne pourrait pas, selon toute apparence, vivre sur les autres planctes. Mais ne doit-il pas y avoir unc infinité d'organisations relatives aux diverses constitutions des globes de cet univers? Si la seule difierence des clémens et des climats, met tant de variécé dans les productions terrestres; combien plus doivent differer, celles des diverses planètes et de leurs satellites. L'imagination la plus active ne peut s'en former aucune idéc; mais leur existence est trés-vraiscmblable

Nous sommes conduits par une forte analogie, à regarder les étoiles, comme aulant de solcils douts ainsi que le nôtre, d'un pouvoir attractif proportionnel à la masse et réciproque au carré des distances. Car ce pouvoir élant démontré pour tous les corps du sysieme solaire, et pour leurs plus petites molécules; il paraît appartenir à toute la matière. Deja, les mouremens des pelites ćtoiles 
SUR LES PROBABILITES.

que l'on a nommées doubles à cause de leur rapprochement, paraissent l'indiquer : un siecle au plus d'observations précises, en constatant lcurs mouvemens de révolution les unes autour des autres, mettra hors de doute, leurs attractions réciproques.

L'analogie qui nous porte i faire de chaque étoile, le contre d'un systime planétaire, est beaucoup moins forte que la précédente; mais clle acquiert de la vraisemblance, par l'hypothese que nous avous proposée sur la formation des étoiles et du soleil; car dans celle hypothése, chaque cloile ayant été comme le soleil, primitivement environnée d'une vaste atmosphère; il est naturel d'attribuer à cette atmosphère, les mêmes effets, qu'à l'atmosphère solaire, ct de supposer qu'elle a produit en se condensant, des planéces et des satcllites.

La méthode la plus sûre qui puisse nous guider dans la recherche de la vérité, consiste a s'élever par la voie de l'induction, des phénomènes particuliers,' à des rapports de plus en plus élendus, jusqu'à ce que l'on arrive enfin à la loi générale dont ils dérivent. Ensuite on vérifie cette loi, soit par des cxpériences directes, lorsque cela est possible, soit en cxaminant si elle satisfait aux phénomenes commus; et si par une rigoureuse 
analyse, on les voit tous découler de celte loi, jusque dans leurs moindres détails; si d'ailleurs ils sont très - variés et très - nombreux; la science alors acquiert le plus haut degré de certitude et de perfection, qu'elle puisse atteindre. Telle est devenue l'astronomie, par la découverte de la pesanteur universelle. L'histoire des sciences fait voir que cette marche lente et pénible de l'induction, n'a pas toujours élé celle des inventeurs. L'imagination impatiente de remonter aux causes, se plaît à créer des hypothises; et souvent, elle dénature les faits, pour les plier à son ouvrage: alors, les hypothèses sont dangereuses. Mais quand on ne les envisage que comme des moyens de lier entre eux les phénomines, pour en découvrir les lois; lorsqu'en évitant de leur attribucr de la réalité, on les rectifie sans cesse par de nouvelles observations; elles peuvent conduire aux véritables causes, ou du moins; nous mettre à porlée de conclure des phénoménes observés, ceux que des circonstances données doivent faire éclore.

Si l'on essayait toutes les hypothises que l'on peul furmer sur la cause des phénomènes; on parviendrait par voie d'exclusion, a la véritable. Ce moyen a été employé arec succés : quelquefois on est arrivé à plusicurs 
hỹpothèses qui cxpliquaient également bien tous les fuits connus, et entre lesquelles les savans se sont partagés, jusqu’à ce que des observations décisives aient fait connaître la véritable. Alors il est intéressant pour l'histoire de l'esprit humain, de revenir sur ces hypothises, de voir comment elles parvenaient a expliquer un grand nombre de faits, et de rechercher les : changemens qu'elles doivent subir, pour rentrer dans celle de la nature. C'est ainsi que le système de Ptolémée, qui n'est que la réalisalion des apparences célestes, se transforme dans l'ly ypothése du mouvement des planctes autour du soleil, en y rendant égaux et parallèles à l'orbe solaire, les cercles et les épicycles que Ptolémée fait décrire anmueliement, et dont il laisse la grandeur, indeterminéc. Il sufici ensuite, pour changer cette hypothese, dans le vrai sy'stème du monde, de transporter en sens contraire, à la terre, le mouvement apparent du soleil.

11 cst presque toujours impossible de soumeltre au calcul, la probabilité des résultats obtenus par ces divers moyens : c'est ce qui a licu pareillement pour les faits historiques. Mais l'ensemble des phénomenes expliqués ou des témoignages, est quelquelois tel, que sans pouvoir en apprcicier la probabilité, on ne peut raisonnablement se permetre aucun 
doutc à leur égard. Dans les autres cas, il est prudent de ne les admettre qu'arec beaucoup de réserve.

Notice historique sur le Calcul des Probabilités.

Depuis long-temps, on a déterminé dans les jeux les plus simples, les rapports des chances favorables ou contraires aux joueurs: les enjeux et les paris étaient réglés d'après ces rapports. Mais personne avant Pascal et Fermat, n'avait donné des principes et des méthodes pour soumettre cet objet au calcul, et n'avait résolu des questions de ce genre, un peu compliquées. C'est donc à ces deux grands géométres qu'il faut rapporter les premiers élémens de la science des probabilités, dont la découverte peut être mise au rang des choses remarquables qui ont illustré le dix-septième siècle, celui de tous les siècles qui fait le plus d'honneur à l'esprit humain. Le principal problème qu'ils résolurent tous deux par des voies différentes, consiste, comme on l'a vu précédemment, à partager équitablement l'enjeu, entre des joueurs dont les adresses sont égales, et qui conviennent de quitter une partie, avant qu'elle finisse; la condition du jeu étant que pour gagner la 
partic, il faut atteindre le premier, un nombre donné de points. Il est clair que le partage doit se faire proportionnellement aux probabilités respectives des joucurs, de gagner cette partic, probabilités qui dépendent des nombres de points qui leur manquent encore. La méthode de Pascal est fort ingénieuse, et n'est au fond, que l'cmploi de l'équation aux différences partielles, relative à ce problème, pour déterminer les probabilités successives des joueurs, en allant des nombres les plus petits aux suivaus. Cette méthode est limitée au cas de deux joueurs : celle de Fermat, fondée sur les combinaisons, s'étend à un nombre quelconque de joueurs. Pascal crut d'abord qu'elle devait être, comme la sienne, restreinte a deux joueurs; ce qui ctablit entre eux, une discussion à la fir de laquelle Pascal reconnut la généralité de la méthode de Fermat.

Huyghens rémit les divers problèmes que l'on avait deja résolus, et en ajouta de nouveaux, dans un petit Traite, le premier qui ait paru sur cette matière, et qui a pour titre, De Ratiocinizs in ludo alea. Plusieurs géomètres s'en occupérent ensuite: Huddes et le grand pensionnaire Witt en Hollande, et Halley en Angleterre, appliquérent le calcul, aux probabilités de la vie humaine; et Halley 
publia pour cet objet, la première table de mortalité. Vers le même temps, Jacques Bernoulli proposa aux géomètres, divers problèmes de probabilité dont il donna depuis, des solutions. Enfin il composa son bel ouvrage intitulé Ars conjectandi, qui ne parut que sept ans après sa mort arrivée en 1706. La science des probabilités est beaucoup plus approfondie dans cet ouvrage, que dans celui d'Huyghens; l'auteur y donne une théorie générale des combinaisons et des suiles, et l'applique à plusieurs questions difficiles, concernant les hasards. Cet ouvrage est cncore remarquable par la justesse et la tinesse des vues, par l'emploi de la formule du binome dans ce genre de questions, et par la démonstration de ce théorème, savoir, qu'en multipliant indéfiniment les observations et les expériences; le rapport des événemens de diverses natures, qui doivent arriver, approche de celui de leurs possibilités respectives, dans des limites dont l'intervalle se resserre de plus en plus, et devient moindre qu'aucune quantité assignable. Ce théorème est très-utile pour reconnaître par les observations, les lois et les causes des phénomines. Bernoulli attachait avec raison, une grande importance à sa démonstration qu'il dit ayoir méditée pendant ringt années. 
Dans lintervalle de la mort de Jacques Bernoulli, à la publication de son ouvrage; Montmort ct Moivre firent paraître deux traítés sur le calcul des probabilités. Celui de Montmort a pour titre, Essai sur les Jeux de hasard: il contient de nombreuses applications de ce calcul, aux divers jeux. L'auteur y a joint dans la scconde édition, quelques lettres dans lesquelles Nicolas Bernoulli donne des solutions ingénieuses de plusieurs problèmes difficiles, de probabilité. Le traité de Moivre, postéricur à celui de Montmort, parut d'abord dans les Transactions Philosophiques de l'année 1711. Ensuite l'auteur Ie publia séparément, et il l'a perfectionné successivement dans les trojs éditions quil en a données. Cet ouvrage est principalement. fondé sur la formule du binome; ct les problèmes qu'il contient, ont, ainsi que leurs solutions, une grande généralité. Mais ce qui le distingue, est la théorie des suites récurrentes, et leur usage dans ces matières. Cette théorie cst lintégration des équations linéaires aux différences finics à coefficiens constans, intégration à laquelle Moivre parvient d'une manière très-heureuse. Comme il est toujours intéressant de connaître la marche des inventeurs; je vais exposer celle de Moivre, en lapplicuant à une suitc récurrente dont la 
relation entre trois termes consécutifs est donnée. D'abord, il considère la relation entre les termes consécutifs d'une progression géométrique, ou l'équation à deux termes, qui l'exprime. En la rapportant aux termes inférieurs d'une unité, il la multiplie dans cet ctat, par un facteur constant, et il retranche le produit, de l'équation primilive. Par la, il obticnt une relation entre trois termes consécutifs de la progression géométrique. Moivre considere ensuite une seconde progression géométrique dont la raison des termes, est le facteur même qu'il vient d'employer. Il diminue pareillement d'une unité, l'indice des termes, dans l'équation de cette nouvelle progression : dans cet état, il la multiplie par la raison des termes de la première progression, et il retranche le produit, de J'équation primitive; ce qui lui donne entre trois termes consécutifs de la seconde progression, une relation enticrement semblable a celle qu'il a trouvée pour la première progression. Puis il obscrve que si l'on ajoute terme à terme, les deux progressions; la même relation subsiste entre trois quclconques de ces sommes consécutives. Il compare les coefficicns de celte relation, à ceux de la relation des termes de la suite récurrente proposće; et il trouvo pour déterminer les rapports des termes 


\section{SUR LES PROBABILTTES.}

consécutifs des deux progressions, une équation du second degré dont les racines sont ces rapports. Par lá, Moivre décompose la suite récurrente, en deux progressions géométriques multipliées, chacune, par une constante arbitraire qu'il détermine au moyen des deux premiers termes de la suite récurrente. Ce procédé est au fond, celui que Lagrange a depuis employé pour l'intégration des équations linéaires aux différences à coefficiens constans.

Trés-peu de temps avant ces recherches de Moivre, Taylor avait donné dans son excellent ouvrage intitulé Methodus incrementorum, la manière d'intégrer l'équation linéaire aux différences du premier ordre, avec un coefficient variable, et un dernier terme fonclion du seul indice. C'est donc à ces deux illustres géomètres, que l'on est redevable de la considération et de l'intégration de ce genre d'équations. A la vérité, les relations des termes'consécutifs des progressions arithmétiques et géométriques, ne sont que les cas les plus simples des équations linéaires aux différences. Mais on ne les avait pas envisagés sous ce point de vue, l'un de ceux qui se rattachant à des théories générales, ont conduit à ces théories, et sont par là, de véritables découvertes. 
Moivre a repris dans son ouvrage, le théorème de Jacques Bernoulli sur la probabilité des résultats donnés par un grand nombre d'observations. Il ne se contente pas de fuire voir, comme Bernoulli, que le rapport des événemens qui doivent arriver, approchera sans cesse de celui de leurs possibilités respectives; il donne de plus une expression élégante et simple de la probabilité que la différence de ces deux rapports, sera contenue dans des limites données. Pour cela, il détermine le rapport du plus grand terme du développement d'une puissance très-élevéc du binome, à la somme de tous ses termes; et le logarithme hyperbolique de l'excès de ce terme, sur les termes qui en sont très-voisins. Le plus grand terme étant alors le produit d'un nombre considérable de factears; son calcul numérique devient impraticable. Pour l'obtenir par une approximation convergente, Moivre fait usage d'un théorème de Stirling sur le terme moyen du binome élevé à une haute puissance, théorème remarquable, surtout en ce qu'il introduit la racine carrée du rapport de la circonférence au rayon, dans une expression qui scmble devoir être étrangère ì cette transcendante. Aussi Moivre fut-il singulièrement frappé de ce résultat que Stirling avait déduil de l'expression de la 
circonférence en produits infinis, expression i laquelle Wallis était parvenu par une singulière analyse qui contient les germes de la théorie si curieuse et si utile des intégrales définies.

Plusicurs savans parmi lesquels on doit distinguer Deparcieux, Kersseboom, Wargentin, Dupré de Saint-Maure, Simpson, Sussmilch, Price et Duviliard, ont réuni un grand nombre de donnécs précieuses, sur la population, les naissances, les mariages et la mortalité. Ils ont donné des formules et des tables relatives aux rentes viagères, aux tontines, aux assurances, etc. Mais dans cette courte notice, je ne puis qu'indiquer ces travaux estimables, pour m'attacher aux idées originales. De ce nombre, est la distinction des espérances mathématique et morale, et le principe ingénieux que Daniel Bernoulli a donné pour soumettre celle-ci ì l'analyse. Telle est encore l'application heureuse qu'il a faite du calcul des probabilités, à l'inoculation. On doit surtout, placer au nombre de ces idées originales, la considération directe des possibilités des événemens, tirées des événemens observés. Jacques Bernoulli et Moivre supposaient ces possibilités, connues; et ils cherchaient la probabilité que le résultat des expériences à faire, approchera de plus en plus de les 
représenter. Bayes, dans les Transactions Philosophiques de l'année 1763, a cherché directement la probabilité que les possibilités indiquées par des expériences déjà faites, sont comprises dans des limites données; et il y est parvenu d'une manière fine et très-ingénicuse, quoiqu'un peu embarrassée. Cet objet se rattache it la théorie de la probabilité des causes et des événemens futurs, conclue des événemens observés; théorie dont j'exposai quelques années apres, les principes, avec la remarque de l'influence des inégalités qui peuvent exister entre des chances que l'on suppose égales. Quoique l'on ignore quels sont les événemens simples que ces inégalités favorisent; cependant cctte iğnorance même accroît souvent, la probabilité des événemens composés. En généralisant l'analyse et les problèmes concernant les probabilités, je fus conduit au calcul des différences finies partielles que Lagrange a traité depuis, par une méthode fort simple, et dont il a fait d'élégantes applications à ce genre de problimes. La théorie des fonctions génératrices, que je donnai vers le même temps, comprend ces objets, parmi ceux qu'elle embrasse, et s'adapte d'elle-même et avec la plus grande généralité, aux questions de probabilité, les plus difficiles. Elle détermine encore par des. 
approximations très - convergentes, les valeurs des fonctions composées d'un grand nombre de termes et de facteurs; et en faisant voir que la racine carrée du rapport de la circonférence au rayon entre lc plus souvent dans ces valeurs, clle montre qu'une infinité d'autres transcendantes peuvent également s'y introduire.

On a encore soumis au calcul, la probabilité des témoignages, les votes et les décisions des assemblées électorales et délibérantes. Tant de passions, d'intérêts divers et de circonstances compliquent les questions relatives à ces objets, qu'elles sont presque toujours insolubles. Mais la solution de problèmes plus simples, et qui ont avec elles beaucoup d'analogie, peut souvent répandre de grandes lumières sur ces questions difficiles.

Liune des plus intéressantes applications du calcul des probabilités, concerne les milicux qu'il faut choisir entre les résultats des obscrvations. Plusieurs géomètres s'en sont occupés, et Lagrange a publié dans les Mémoires de Turin, une belle méthode pour déterminer ces milieux, quand la loi des erreurs des observations est connue. J'ai donné jour le même objet, une méthode fondée sur un artifice singulierqui peut être employé avec avantage dans d'autres questions d'analyse, 
et qui en permettant d'étendre indéfiniment dans tout le cours d'un long calcul, les fonctions qui doivent être limitées par la nature du problème, indique les modifications que chaque terme du résultat final doil recevoir en vertu de ces limitations. Mais ces méthodes supposent connuc, la loi des crreurs des observations; ce qui n'est pas. Heureusement, j’ai trouvé que si les observations sont. cn grand nombre, la recherche des milieux que l'on doit choisir, devient indépendante de celte loi. On a vu précédemment, que chaque obscrvation fournit une équation de condition, du premier degré, qui peut toujours être disposée de manière que tous ses termes soient dans le premier membre, le second étant zéro. L'usage de ces équations est une des causes principales de la grande précision de nos tables astronomiques; parce que l'on a pu ainsi faire concourir un nombre immense d'excellentes observations, à la détermination de leurs élémens. Lorsqu'il n'y a qu'un seul élément à délerminer, còles avait prescrit de préparer les équations de condition, de sorte que le coefficient de l'élément inconnu tùt positif dans chacune d'elles, et d'ajouter ensuite toutes ecs équations, pour former une équation finale d'où l'on tire la valeur de cet élément. La règle de Cótes 
fut suivie par tous les calculateurs. Mais quand il fallait déterminer plusieurs élémens; on n'avait aucume règle fixe pour combiner les équations de condition, de manière à obtenir les équations finales nécessaires: seulement, on choisissait pour chaque élément, les observalions les plus propres a le déterminer. Ce fut pour obvier à ces tâtonnemens, que Legendre et Gauss imaginirent d’ajouter les carrés des premiers membres des équations de condition, et d'en rendre la somme un minimum, en y faisant varier chaque élément inconnu : par ce moyen, on obtient directement autant d'équations finales, qu'il y a d'ćlémens. Mais les valeurs déterminées par ces équations, méritent-elles la préférence sur toutes celles que l'on peut obtenir par d'autres moyens? C'est ce que le calcul des probabilités pouvait seul apprendre. Je l'appliquai donc à cet objet important, et je fus conduit par une analyse délicate, à la règle que je viens d'indiquer, et qui réunit ainsi à l'avantage de faire connaître par un procédé régulier, les élémens cherchés, celui d'en donner les valeurs qui ne laissent à craindre que les plus petites erreurs possibles.

J'ai rassemblé tous ces objets dans ma Théorie analylique des Probabilités, ou je me suis proposé d'exposer de la manière la 
plus générale, les principes et l'analyse du calcul des probabilités, ainsi que les solutions des problèmes les plus intéressans et les plus difficiles que ce calcul présente.

On voit par cet Essai, que la théorie des. probabilités n'est au fond, que le bon sens réduit au calcul : elle fait apprécier avec exactitude, ce que les esprits justes sentent par une sorte d'instinct, sans qu'ils puissent souvent s'en rendre compte. Si l'on considere les méthodes analytiques auxquelles cette théorie a donné naissance, la vérité des principes qui lui servent de base; la logique fine et délicate qu'exige leur emploi dans la solution des problèmes, les établissemens d'utilité publique qui s'appuient sur elle, et l'extension qu'elle a reçue et qu'elle peut recevoir encore, par son application aux questions les plus importantes de la philosophie naturelle et de l'économie politique; si l'on observe ensuite que dans les choses mêmes qui ne peuvent être soumises au calcul, elle donne les aperçus les plus sûrs qui puissent rous guider dans nos jugemens, et qu'elle nous apprend à nous garantir des illusions qui souvent nous égarent; on verra qu'il n'est point de science plus digne de nos méditations, et dont les résultats soient plus utiles. 


\section{TABLE DES MATIËRES.}

ESSAI PHILOSOPIIQUE SUR LES PROBABILITES Page 1

De la Probabilité.................... 2

Principes généraux du Calcul des Probabilités. 12

De l'Esperance.................... 23

Des Methodes analytiques du Calcul des Proba-

bilites...................... 30

\section{Applications du calcul des probabilités.}

Des Jeux.

Des inégalités inconnues qui peuvent exister entre des chances que l'on suppose égales........ 61 De la Probabilité des témoignages........... 65 Des choix et des decisions des assemblées.... 87 Des Lois de la Probabilité, qui résultent de la multiplication indéfinie des évenemens..... g3 Du Calculdes Probabilités, appliqué à la recherche des phénomènes et de leurs causes......... 107 Des milieux quil faut choisir entre les résultats d'un grand nombre d'observations......... 127 Des Tables de mortalité, et des durees moyennes de la vie, des mariages et des associations quelconques.................... 135

Des benéfices et des établissemens qui dépendent de la Probabilité des événemens.......... 146 Des illusions dans l'estimation des Probabilités. 154 Des divers moyens d'approcher de la certitude.. 168 Notice historique sur le calcul des Probabilites. $17^{8}$ 


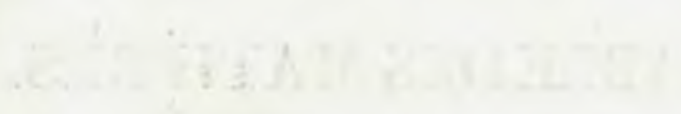

y $=2+2$

………… 





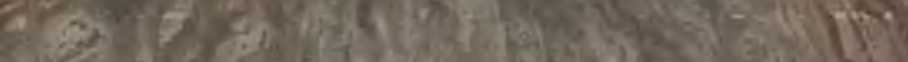
(2)

mos

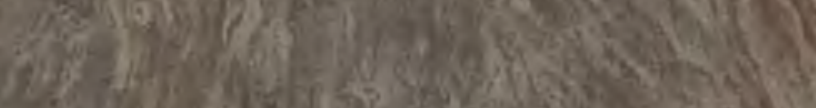

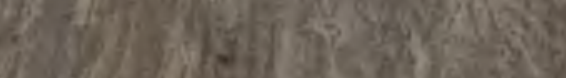

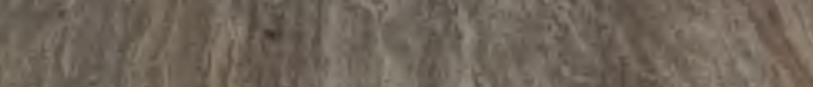

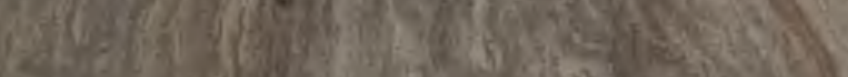

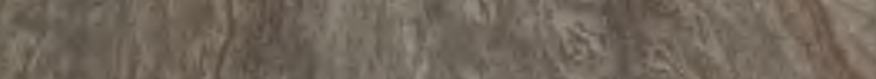

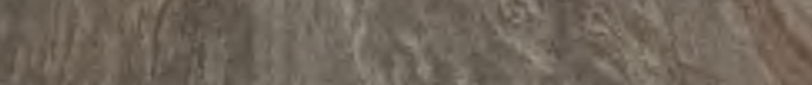
Le.

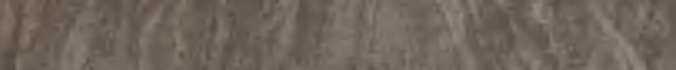

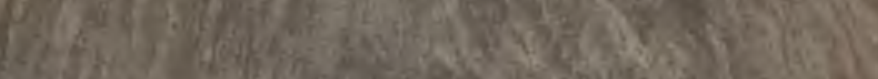

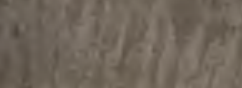

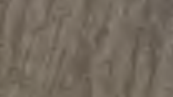

(is)

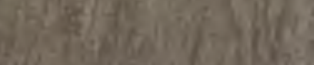

$\operatorname{lig}_{i} \operatorname{lin}^{2}$

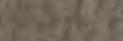

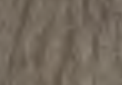

(iit)

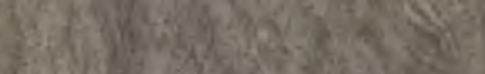

of dos.

istit.

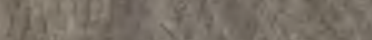

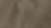

is?

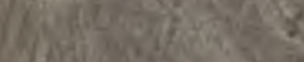

I. Wow

(1)

$\frac{1}{4} x^{2}$

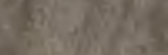

(a) $\left.\frac{2}{4}\right)^{n}=\frac{2}{2}$

$\frac{3}{4}$

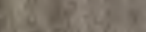

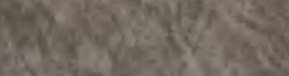

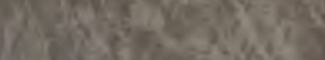

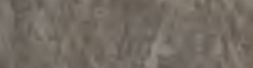

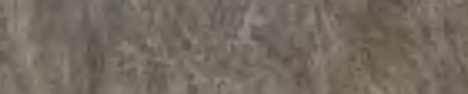

$$
\text { ( }
$$

